\title{
Introduction
}

\section{The choice of, and the decision to apply, disease modifying drugs}

\author{
COLIN G BARNES \\ From The London Hospital, London E1, UK
}

\begin{abstract}
Although rheumatoid arthritis (RA) is recognised to be a systemic illness, frequently the only manifestation is an inflammatory polyarthritis in which synovitis causes joint destruction. Extra-articular or systemic features include vasculitis which, when present, may be the cause of potentially serious or fatal manifestations. The aetiology remains unknown, although immunological disturbances are certainly involved in the perpetuation of the disease. The formation of immune complexes within the joint is probably responsible for the release of chemical mediators of inflammation.

The natural history of the disease is variable, ranging from a brief episode of polyarthritis which resolves spontaneously, to a rapidly progressive arthritis with widespread systemic features.

The evidence for an immunological disturbance arises from:

(1) the presence of serum and synovial fluid autoantibodies - rheumatoid factors;

(2) the presence of other serum antibodies, for example antinuclear antibodies;

(3) the histology of the synovium which includes infiltration with plasma cells and lymphocytes;

(4) reduced synovial fluid complement levels in actively inflamed joints;

(5) the presence of immune complexes in synovial fluid;

(6) reduced serum complement levels in patients with active systemic disease;

(7) amyloid deposition.
\end{abstract}

The first line of treatment always consists of the use of non-steroidal anti-inflammatory analgesics, in full dosage, to achieve both pain relief and some reduction of the inflammatory component of the synovitis. There is, however, no evidence that these drugs influence the natural history of the disease, and therefore further drugs are continually being sought which will modify the disease process and suppress the synovitis and vasculitis in particular. Such drugs which are available at present are potentially toxic and have not been shown predictably to halt the progression of the disease. Thus their use, in a disease which is usually not fatal, depends on the careful assessment of the course of the disease in the individual patient and on the making of a balanced judgement between the likely effect of the disease, if not suppressed, and the possible toxicity of the treatment.

The decision to use a drug which may suppress the disease, drugs which are also known as 'slow-acting', 'long-acting' or 'second-line' agents, depends on a definite diagnosis with evidence that the disease is progressing. Evidence of such progression may be demonstrated by some or all of the following features:

(1) persistent active synovitis

- prolonged morning stiffness

- pain, warmth and tenderness of joints

- clinical synovitis;

(2) deteriorating functional capacity owing to active inflammatory synovitis;

(3) increasing number of affected joints;

(4) rheumatoid factor tests -becoming positive

-increasing titre;

(5) radiological erosions

-development or increase in
number;

(6) development of extra-articular features, for example nodules, vasculitis, etc.

The group of suppressive drugs consists of seven different classes of agents:

(1) gold salts;

(2) antimalarials-chloroquine preparations;

(3) 'immunosuppressives' (cytotoxic: antimetabolic agents); 
(4) penicillamine;

(5) levamisole;

(6) dapsone;

(7) sulphasalazine.

Of these, the first four are well established in the treatment of RA. Levamisole has been shown to be effective in a majority of patients but is considered by many to produce too high an incidence of toxic effects to be considered for routine treatment. ${ }^{98} 116209229$ The effectiveness of dapsone and sulphasalazine remains debatable. ${ }^{135-137}$

Historically gold salts are the most firmly established form of suppressive treatment of RA, having been introduced over 50 years ago by Forrestier. It was not until controlled trials were conducted by Fraser $^{70}$ and the Empire Rheumatism Council ${ }^{63}$ that its effectiveness was demonstrated. Nevertheless, its mode of action remains uncertain, prediction of both benefit or toxicity in the individual patient is not possible and measurement of serum levels has not been proved to be a useful method of monitoring gold therapy..$^{100} 188$

The chloroquine group of drugs were added next, initially being used empirically but later being shown to be effective in controlled trials, ${ }^{87} 139180$ but less effective than gold or azathioprine. ${ }^{56}$

The first report of the use of a cytotoxic agent in the management of RA was in 1951, when JimenezDiaz $^{106}$ treated nine patients with nitrogen mustard on the basis that it was appropriate to treat proliferative synovitis with an antiproliferative agent. There followed other reports of the use of this agent, but by 1964 interest had moved to the so-called immunosuppressives, in particular azathioprine, chlorambucil and cyclophosphamide. The rationale for the use of these agents was that if an immunological disturbance plays an important part in the pathogenesis of RA, then it would be beneficial for this immunological overactivity to be suppressed. Thus, by 1972 there were 25 published trials of azathioprine in 350 patients, nine trials of chlorambucil in 330 patients, and 12 trials of cyclophosphamide in 370 patients, and an additional 235 patients had been treated in trials with aminopterine, 6-mercaptopurine or methotrexate. The majority of these trials were uncontrolled. ${ }^{14}$ However, there have now been many controlled trials, mainly of azathioprine and cyclophosphamide, which have demonstrated their efficacy and their toxicity, which have compared them one with the other and also with gold salts, chloroquine and penicillamine, and which have compared different dosage schedules. ${ }^{5} 1834353856848995124143224225237239$ Nevertheless, it has not been convincingly demonstrated that immune responses are suppressed in vivo. Of particular concern is the debate on the possible oncogenic effect of the immunosuppressive cytotoxic agents, ${ }^{5}$ although to date a large follow-up survey has only revealed a small increase in the number of non-Hodgkin's lymphomata in patients who are not organ transplant recipients. ${ }^{115}$

Most recently penicillamine has been studied, the results of the first series of 21 patients being published by Jaffe in $1965 .{ }^{103}$ Although the mode of action of penicillamine remains unknown, controlled trials have shown it to be effective and it has been subjected to trials of different dosage regimens and in comparison with other suppressive agents. This drug too is potentially toxic. ${ }^{18489799133155}$

None of the agents available at present provides a complete answer to the need to suppress RA, in that approximately $75 \%$ of patients respond to treatment and certainly a complete cure is not effected. The measurement of efficacy depends on clinical response, improvement in laboratory parameters of disease activity and, possibly most importantly, the demonstration of a reduction in progressive joint damage. Only the 'immunosuppressive' cytotoxic agents have been shown possibly to reduce the progression of radiological erosive change,$^{34}{ }^{38}$ and a histological study of the effect of chlorambucil on active synovitis did not reveal any significant improvement. $^{5}$

This symposium, therefore, aims to re-examine our present state of knowledge of the effectiveness and toxicity of the drugs currently available which may modify the rheumatoid process, and of the 'immunosuppressive' cytotoxic agents in particular.

Questions which may be posed are:

(1) May these drugs be considered to modify the progression of the disease by:

(a) suppressing the activity of the inflammatory component of rheumatoid arthritis?

(b) reducing or preventing the progression of erosive joint damage?

(c) suppressing the laboratory measurements of inflammation and immunological overactivity?

(2) What is the long term toxicity of these agents?

(3) What is the comparative efficacy and toxicity of the different agents?

(4) What are appropriate dosage schedules?

(5) What are their modes of action?

We shall consider both clinical and laboratory data and hope for a wide-ranging discussion between the speakers and the audience. 


\title{
Immunosuppression and the rheumatic diseases
}

\author{
A M DENMAN
}

From the Connective Tissue Diseases Study Group, Clinical Research Centre, Northwick Park Hospital,
Watford Road, Harrow, Middlesex, England

SUMMARY Ignorance of the basic nature of rheumatoid arthritis precludes the introduction of rational schemes for using cytotoxic drugs. It is still plausible that the autoimmune and other immunological abnormalities which accompany this disease are the secondary effects of persistent antigen, for example, related to microbial infections. In this event, cytotoxic drugs may diminish the inflammatory response but their effects on immune responses would be irrelevant or even undesirable. Should rheumatoid arthritis prove to be a primary immunoproliferative disorder, cytotoxic drugs may prove to be of value not because of their conventional immunosuppressive effects but because of their selective action on the proliferating cells. Indeed, current evidence suggests that these drugs enhance rather than depress conventional immune responses, at least in the doses given to patients with rheumatic disorders.

Cytotoxic drugs were introduced into rheumatological practice because of rather simplistic views about the pathogenesis of these disorders. The basic abnormality was considered to be a loss of tolerance to self-antigens, allowing the proliferation of abnormal clones of lymphocytes with selfreactivity. ${ }^{210}$ Cytotoxic drugs, it was thought, might eliminate these clones thereby terminating autoimmune diseases such as rheumatoid arthritis and systemic lupus erythematosus. Furthermore, there was experimental evidence that drugs such as cyclophosphamide might aid and abet the reestablishment of tolerance. The situation is now recognised to be far more complicated. There is no longer such confidence that these diseases can be explained by simple unitarian theories, because autoimmune phenomena can be induced by a variety of mechanisms and need not reflect the basic pathogenetic process in these diseases. Moreover, cytotoxic drugs and other immunosuppressive agents do not have a simple dose-related effect on immune responses, a possibility which earlier knowledge of immunological mechanisms could not take into account.

It is now accepted that conventional and autoimmune reactions involve the interactions of several populations of lymphocytes and accessory cells, both with varying sensitivity to immunosuppressive agents. Above all, there are populations of lymphocytes which control the nature and magnitude of all immune responses whether these are mediated by lymphocytes, antibodies, or even by non-specific 'natural killer' (NK) cells. Thus immunosuppressive drugs, which interfere selectively with the control of immune responses, may increase the vigour of such responses if the cells which effect these responses are themselves resistant to the agent in question.

There are additional difficulties because the causes of most inflammatory connective tissue diseases are unknown. Until recently techniques for assaying immune function in clinical practice have been crude and inexact. Few clinical studies have taken pharmacokinetic factors into consideration when assessing the effects of cytotoxic drugs on immune reactions. It is hardly surprising, therefore, that there should be little reliable information about the crucially important ways in which immunosuppressive agents affect the clinical course of these diseases.

\section{Persistent antigens and connective tissue diseases}

Logic, rather than practical results, still inspires the belief that persistent inflammation in the synovial membrane and other tissues affected by rheumatic disorders results from persistent antigen. Quite apart from the obvious interest in efforts to isolate the provoking 'agent', this approach has important implications for interpreting the immunological aberrations which abound in the rheumatic diseases, and thus for the correct line of treatment. If a disease such as rheumatoid arthritis is induced by persistent antigen, the disorder should logically be regarded as a form of immunodeficiency because of the host's inability to eradicate the causative agent. The arthritis and autoimmune features which accompany gross immunodeficiency are often attributable to specific infections. This arouses the suspicion that more specific forms of immunodeficiency to precisely defined organisms might account for similar disorders in patients without evidence of general immunodeficiency. ${ }^{10}$

Many of the causes of antigen persistence are also 
liable to produce immunological abnormalities. These are of two kinds, the first being depressed immune responses to the antigen itself, and the second being indirect in nature. For example, one postulated source of persistent antigen is bacterial cell wall products which escape immune elimination or enzymatic degradation. ${ }^{17}$ Bacterial cell walls, or peptidoglycans isolated from this source, induce a variety of inflammatory lesions in experimental animals, the distribution of which variously resembles rheumatoid arthritis or diffuse inflammatory connective tissue diseases. In addition, bacterial cell wall peptidoglycans stimulate a brisk proliferative response by lymphocytes and this is usually matched by equivalent antibody response. ${ }^{117}$ Nevertheless, bacterial infections may fail to induce an appropriate antibody response. One notable example is provided by experimentally infecting mice with living or killed cocci. ${ }^{83}$ The infected mice show a severely impaired immune response against the cell wall antigens in the invading micro-organisms. In addition, the infected mice display a variety of immunological aberrations which closely resemble those observed in patients with rheumatic disorders. These include a diminished capacity to react against other antigens, the production of autoantibodies resembling rheumatoid factor and the generation of antibodies to bacterial cell wall glycoproteins which cross react with lymphocyte membrane glycoproteins. These lymphocyte reactive autoantibodies are analogous to lymphocytotoxins, and show a restricted specificity for those lymphocyte antigens which are displayed only during the nonproliferative phase of the cell cycle. A wide spectrum of autoantibodies is also observed in experimental animals with chronic parasitic infections such as trypanosomiasis. ${ }^{119}$

Virus infections of man and experimental animals also induce a varying range of immunosuppressive effects and immunological aberrations. ${ }^{43} \mathrm{~A}$ number of mechanisms contribute to these effects. In man the most important of these is the ability of viruses to grow in sub-populations of those lymphocytes which are essential for the induction of specific immune responses. ${ }^{173}$ There is little evidence that virus infections in man are potentiated by the ability of the invading virus to inactivate the specific host immune response to the virus in question. However, there are human diseases in which specific immune defects do predispose to an unusually severe or atypical infection. A notable example is the wide range of immunoproliferative disorders induced by EpsteinBarr (EB) virus infections in patients with preexisting defects in specific cell mediated immunity to this virus, or possibly with defects produced by the infection itself. Thus, immunological aberrations which may result from persistent infection include impaired immune responses, hypergammaglobulinaemia, rheumatoid factor and autoantibodies of varying specificity, all of which have often been considered to be characteristic of primary autoimmune disease. This conclusion has prompted the therapeutic assumption that the suppression of such abnormalities will ameliorate the disease. However, if these abnormalities are the result, rather than the cause, of the inflammatory lesions in diseases such as rheumatoid arthritis, there are different therapeutic implications. Obviously, simply correcting these defects would have little relevance to the primary cause of the inflammatory lesion. Equally obviously, the most appropriate way of correcting these abnormalities would be to remove the source of persistent antigen, but this can rarely be achieved. Nevertheless, it is pertinent to this argument that immunological aberrations are observed in patients with sub-acute bacterial endocarditis, including hypergammaglobulinaemia and the production of rheumatoid factor and antinuclear antibodies, but these abnormalities disappear in patients whose disease responds to antibiotics. Similarly, the range of autoimmune phenomena provoked by drugs may equal that observed in patients with systemic lupuso erythematosus of unknown cause, and theseo immunological abnormalities also resolve once the offending drug has been withdrawn. ${ }^{235}$

Given that the object of immunosuppressive treatment in most patients with rheumatic diseases is to control the disease process rather than to eliminate the cause, one can question the extent to which such treatment can be expected to provide therapeutic benefit if the immune aberrations are of secondary importance. There are a number of ways in which cytotoxic drugs, and other immunosuppressive agents, can blunt the inflammatory changes induced by antigen persistency. In general terms these involve the depression of inflammatory reactions which are set in train by specific immune events and are too well-known to need further description. These involve humoral mechanisms with detectable circulating immune complexes, and a variety of cellular events mediated by granulocytes, cells of the monocyte-macrophage series, 'natural killer' cells and $\mathrm{K}$ cells. Experimental observations in man testify to the suppressive effects of these drugs on inflammatory reactions and, perhaps the most telling observation of all, in the frequency of opportunistic microbial infections and the reactivation of viral infections consequent upon such treatment.

Nevertheless, some fundamental questions remain unanswered. The first concerns the extent to which cytotoxic drugs really reinforce the effects of steroids on these inflammatory events, or whether they have a more fundamental effect on the immunological reac- 
tions which induce the inflammation. It is in this context that the complexity of immune responses makes this a difficult question to answer. A variety of suppressor cells have been detected in man that could interfere with efficient responses to persistent antigens. For example, a sub-population of $\mathrm{T}$ lymphocytes interferes with natural cytotoxicity against virus-transformed cell lines normally mediated by NK cells. ${ }^{41}$ There is good evidence also that various suppressor cell populations are activated in human hosts chronically infected with different microbial infections. ${ }^{217}$ Thus the inability to eliminate the putative persistent agent in a disease such as rheumatoid arthritis could also result from suppressor cells interfering with the generation of an efficient cellmediated immune response against persistently infected cells. There is, for example, good evidence that delayed hypersensitivity reactions to soluble proteins such as ovalbumin and bovine gammaglobulin are controlled by suppressor T lymphocytes. Cyclophosphamide ablates this suppressor mechanism, thereby producing exaggerated delayed hypersensitivity responses to these antigens. ${ }^{168}$ The effects of cytotoxic drugs in connective tissue diseases are, therefore, unpredictable. Logically, these drugs could overcome a defective immune response to a persistent antigen and improve responses to unrelated antigens in addition.

\section{The effects of immunosuppressive agents on immunoproliferative diseases}

The clonal theory of autoimmune diseases has been strengthened by recent observations concerning the normal generation of immune responses. It is now apparent that immune responses to foreign antigens are initiated by $\mathrm{T}$ lymphocytes which see such antigens in combination with self-antigens. $T$ lymphocytes destroy target cells bearing foreign antigens most efficiently when the target cells and the T lymphocytes concerned in their destruction have histocompatability antigens in common. Indeed it is now proposed that the principal function of histocompatability antigens is to expedite the surveillance function of $\mathrm{T}$ lymphocytes. ${ }^{160}$ Autoimmune reactions, therefore, are no longer viewed as qualitatively different totally abnormal responses, compared with conventional responses, to exogenous antigens; it is the capacity of autoreactive cells to escape normal regulatory mechanisms which determines whether or not such responses will predominate. This concept has been strengthened by repeated observations that autoreactive $B$ lymphocytes are found in normal subjects. ${ }^{210}$ These regulatory defects were initially thought to involve control mechanisms such as suppressor $\mathrm{T}$ lymphocytes and the capacity of the normal immune system to generate antibodies with specificity for unique $\mathrm{V}$ region sequences (idiotypes) in the immunoglobulin molecule. More recently it has been established that the generation of antibody diversity in B lymphocytes involves a number of recombination steps between genes coding for different portions of the heavy and light chain molecule. Thus, there is great potential for errors in the sequence of events by which B lymphocytes synthesise antibodies of different isotypes and of different specificities. Consequently, more attention has been given to the possibility that autoreactive $\mathrm{B}$ lymphocytes may proliferate in an uncontrolled manner, because of errors in the differentiation of the precursor stem cells for these B cells or in their maturation. ${ }^{50}$

So far, the clonal origin of lymphoproliferative diseases has been established only in malignant forms, such as chronic myeloid leukaemia and polycythaemia vera. To some extent, cytogenetic and immunochemical analysis of both membrane-bound and secreted immunoglobulins will establish this point. However, the point can be made most clearly in black females who are heterozygous for the Xlinked enzyme glucose-6-phosphate dehydrogenase and who developed myeloproliferative diseases. ${ }^{67}$ The cellular origin of these disorders can be examined in detail in such subjects. B lymphocytes in diseases such as chronic myeloid leukaemia and polycythaemia vera are derived from a single abnormal malignant clone even though these B lymphocytes produce immunoglobulins with the full repertoire of heavy and light chain determinants, a property previously considered to be characteristic of $\mathrm{B}$ cells of polyclonal origin. ${ }^{142}$ It has also been clearly established that the autoantibodies in some patients with cold agglutinin disease and haemolytic anaemia are also of clonal origin. There is an attractive possibility, therefore, that the apparently polyclonal antibodies in other common autoimmune diseases, such as rheumatoid arthritis and systemic lupus erythematosus, may also be produced by $\mathrm{B}$ lymphocytes derived from a limited number of progenitor stem cells. By this concept, rheumatic disorders would be considered as primary lymphoproliferative disorders, and this would explain the failure to find the postulated persistent antigens at sites of chronic inflammation. So far there is no firm evidence of a similar nature in immunoproliferative diseases. Nevertheless, there are many striking similarities between the immunological features of the rheumatic diseases and those detected in murine models of human autoimmune disease..$^{50}$ The immunopathological events in these mice have been intensively studied and the primary defect involves the differentiation and maturation of the $B$ cell progeny of bone marrow stem cells. Moreover NZB mice at 
first display a polyclonal hypergammaglobulinaemia, but monoclonal paraproteins subsequently emerge. This process can be correlated with the emergence of a single abnormal clone, detectable cytogenetically, whose progeny eventually infiltrate the spleen and lymphoid system.

There have been many attempts to demonstrate that there is a fundamental abnormality in the regulation of immune responses in patients with rheumatic diseases. Most attention has been directed at demonstrating a defect of suppressor $\mathrm{T}$ lymphocytes and abnormalities have been reported in patients with systemic lupus erythematosus and their relatives. Until recently, less attention has been given to seeking defects in B lymphocyte function. However, it is now clear that such abnormalities exist. Cultured B lymphocytes from patients with systemic lupus erythematosus spontaneously synthesise large amounts of immunoglobulin in vitro, but are refractory to pokeweed mitogen, a mitogen which normally induces polyclonal immunoglobulin synthesis by human B lymphocytes. Moreover, patients with this disorder show impaired in vitro and in vivo antibody synthesis in response to immunisation with tetanus toxoid. ${ }^{159}$ Techniques are now available for analysing the control of specific antibody synthesis in vitro. Recent observations ${ }^{172}$ have shown that whereas the lymphocytes from normal subjects produce antibody after in vitro challenge with influenza viral antigen, lymphocytes from patients with systemic lupus erythematosus fail to respond in this manner. Experiments in which isolated populations of $\mathrm{T}$ and $\mathrm{B}$ lymphocytes from lupus patients and from normal controls were co-cultured in vitro show that the defect resides in the B lymphocyte population. Thus B lymphocytes from lupus patients were able to function normally as 'helper' cells in specific antibody synthesis, but B lymphocytes from lupus patients were still defective even when combined with 'helper' $\mathrm{T}$ cells from normal donors. One can propose that systemic lupus erythematosus is an oligoclonal lymphoproliferative disorder in which B lymphocytes with memory for previous immunological events express some conventional antibody response, but the majority of immunoglobulin synthesis is programmed to produce autoantibodies. Speculation continues about the basic defect which could initiate this uncontrolled proliferation. There are many, admittedly incomplete, hints about the possible nature of these initiating insults. Drugs which bind DNA may induce a syndrome resembling spontaneous systemic lupus erythematosus, and subjects who acetylate such drugs poorly are particularly prone to develop this complication. ${ }^{16}$ There is also a clinical impression that intercurrent virus infections exacerbate inflammatory connective disorders. It is there- fore possible that viruses and drugs act as promutagens, that is, agents which bind to DNA and induce mispairing with other bases. ${ }^{122}$ If not excised by the appropriate excision and repair enzymes, persistence of promutagens could, in proliferating lymphocytes, produce mutations coding for abnormal immunoglobulins with autoreactive specificities. There are indications that lymphocytes from patients with inflammatory connective tissue diseases, including rheumatoid arthritis, are abnormally susceptible to promutagens such as alkylating agents and lowdose ultraviolet irradiation, and this sensitivity appears to be related to a deficiency in removing alkylated bases from lymphocyte DNA. ${ }^{88}$

Should inflammatory connective tissue diseases prove to result from primary immunoproliferative diseases, this would have clear implications for the ways in which immunosuppressive agents might operate. Under these circumstances the aim of treatment would be to eradicate abnormal autoantibody producing cells in a manner analogous to the treatment of malignant lymphoproliferative diseases. Moreover, drugs such as cyclophosphamide and azathioprine would be more likely to suppress these cells rather than lymphocytes producing conventional antibodies, since such lymphocytes areo highly resistant to cytotoxic drugs. Indeed the resistance of conventional secondary antibody responses, but sensitivity of autoantibody production to cytotoxic drugs, points to this conclusion.

\section{Clinical observations in patients receiving immuno- suppressive agents}

Considerable information has accumulated concerning the effects of immunosuppressive agents on various immunological functions. Unfortunately, the results have been conflicting and confusing. Such criticisms particularly concern attempts to assay the in vitro function of lymphocytes isolated from patients receiving immunosuppressive drugs. Technical problems account for some of the discrepancies in published results. Most investigators have carried out tests of lymphocyte function in vitro using a single concentration of cultured cells and a standard mitogen challenge. However, it is now clear that the conditions for detecting peak responses to mitogenic stimuli vary in different diseases and are affected by many secondary factors. ${ }^{65}$ Another problem is the understandable failure of earlier workers to appreciate the complexities of immune responses. It is now apparent that meaningful assays of immune function must include tests for all the populations of inflammatory cells which contribute to tissue damage and which modulate these effects. Two principal strategies are being introduced. The first relies on the 
ability of monoclonal antibodies to identify suitable markers of each function of sub-populations of lymphocytes, and to score these both in the peripheral blood and in the inflammatory lesions of patients with rheumatic disorders. The second involves functional assays, such as specific antibody production, and, of equal importance, suppressor cell activity. So far there is too little evidence to make dogmatic statements about the extent to which different immunosuppressive agents, or regimens, have selective effects on the different cell populations and immune mechanisms which contribute to the disease process. Nevertheless, there are already firm indications that theoretical immunosuppressive regimens do not predictably suppress conventional immune responses as judged by current standard techniques. Indeed, most observations of standard immune responses in such patients have indicated that these responses were as often increased as depressed by cytotoxic drugs. Paradoxically, too, immunosuppressive treatment commonly improves bone marrow function and host responses to infection in subjects with bone marrow depression and immunodeficiency attributable to connective tissue diseases. This point is emphasised by experience in a comparative trial in which patients with systemic lupus erythematosus, polyarteritis, Behçet's syndrome, or dermatomyositis have been treated with steroids alone in conventional doses, or with a combination of high dose steroid, antilymphocyte globulin and cytotoxic drugs. ${ }^{44}$ Contrary to expectations, bone marrow function and resistance to infection improved more rapidly in patients receiving the more intensive regimen. This point is illustrated by two clinical examples.

The first (fig 1) is a 16-year-old patient with systemic lupus erythematosus, presenting with grand-mal fits and skin lesions, with clinical cutaneous vasculitis, hypertension, hepatosplenomegaly and progressive renal disease. He had strongly positive tests for antinuclear antibody, circulating immune complexes in high titre and hypocomplementaemia. He failed to respond to steroids in high dosage (up to $100 \mathrm{mg}$ prednisone daily) and developed congestive heart failure and staphylococcal septicaemia. Treatment with intensive immunosuppression produced a full clinical remission and the disappearance of most of the characteristic serological abnormalities. However, it is noteworthy that his haemoglobin and white cell count also returned to normal on treatment. Depressed lymphocyte function, which had been detected in vitro associated with his disease, also reverted to normal on allegedly immunosuppressive treatment.

The second case is an 18-year-old boy who developed polyarteritis with a mononeuritis multiplex, vasculitis leading to digital ischaemic changes

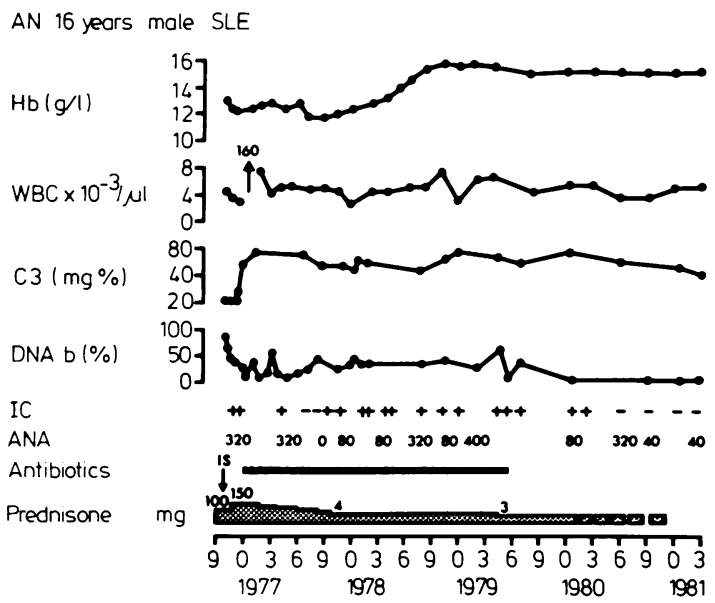

FIG 1 Bone marrow recovery in a patient with systemic lupus erythematosus receiving intensive immunosuppression. (C3=complement, $D N A=D N A$ binding assay, $I C=$ immune complexes, $A N A=$ antinuclear antibody reciprocal titre.)

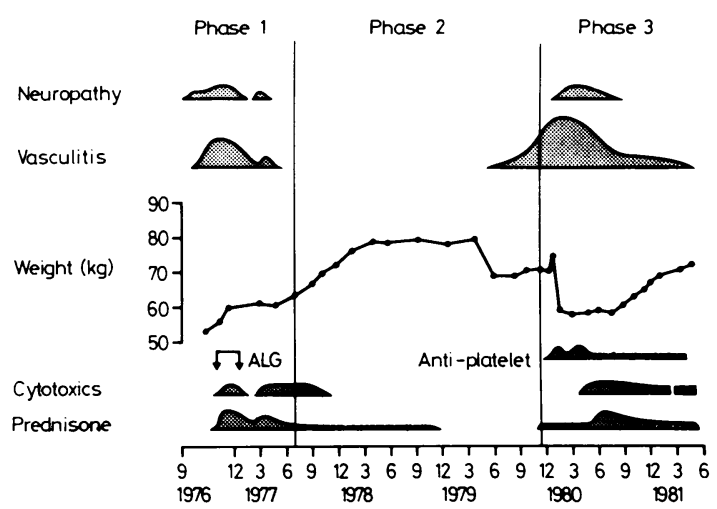

FIG 2 Response of polyarteritis to immunosuppressive treatment. The three phases of the disease are described in the text. Cytotoxics: azathioprine and cyclophosphamide; prednisone: $150 \mathrm{mg} /$ day maximum; anti-platelet: aspirin. sulphinpyrazone, tolazoline. ALG=antilymphocyte globulin.

and renal disease (fig 2). The first phase of his illness was characterised by severe clinical disease which responded to immunosuppression with high dose steroids (maximally $150 \mathrm{mg}$ prednisone daily), azathioprine, cyclophosphamide and antilymphocyte globulin (ALG). His disease went into remission and all drugs were withdrawn (phase 2). However, the vasculitic lesions and neuropathy reappeared although, at this stage (phase 3), the lesions were characterised histologically by an obliterative endarteritis rather than by inflammatory 
vasculitis. He eventually recovered with further treatment with high dose steroids and cyclophosphamide. It was noteworthy that resistance to secondary infections accompanied periods of active disease but were controlled once adequate immunosuppressive regimens were instituted.

These findings do not help us to distinguish between the two major hypotheses explaining the clinical and immunological abnormalities of the rheumatic diseases. Improved conventional immune responses and the disappearance of immunological anomalies, such as autoantibody production, would be expected if cytotoxic drugs corrected the abnormalities consequent on antigen persistence, for example, in association with persistent infection. Equally, if cytotoxic drugs eliminate clones of autoantibody producing cells which are particularly sensitive to these drugs, the proliferation and maturation of B lymphocytes derived from normal stem cells might be improved, thereby allowing conventional immune responses to recover. The immunodeficiency associated with malignant myeloproliferative diseases, such as the leukaemias, is indeed often reversed as the result of chemotherapy which has been regarded as a favourable prognostic sign.

\section{Conclusions}

Despite many years of treatment with cytotoxic drugs, no clear picture has emerged of the ways in which these drugs influence inflammatory connective tissue diseases. The issue is therapeutically important since, if steroids produce their undoubted beneficial effect through immunosuppressive mechanisms, then any drugs which potentiate those effects should be clinically beneficial. Ironically, rapid improvements in our knowledge of the pharmacological events in inflammation have called in question the belief that the therapeutic benefits of steroids owe anything to their effects on lymphocytes and immune function. Indeed it is arguable that the latter are undesirable side effects of steroid treatment. Given our ignorance of the pathogenesis of these diseases and the many plausible ways in which cytotoxic drugs could produce benefit, it is hardly surprising that the issues remain unresolved. The quickest way to resolve the dilemma will be to elucidate the pathogenesis of these diseases. In the meantime, technical improvements in assaying responses in clinical practice at least allow the prospects of pursuing meaningful studies concerning the effects of cytoxic drugs on immune function. 


\title{
Differences among immunosuppressive agents
}

\author{
GEORGE H HITCHINGS \\ From the Wellcome Research Laboratories, Research Triangle Park, NC 27709, USA
}

SUMMARY Immunosuppressive agents have diverse (although often multiple) sites of action in the cell sequences that are involved in immune responses. New routes to selectivity are apparent at both the cellular and the biochemical level. Meanwhile, clinical work is finding new uses and more selective employment of the currently available agents.

Immunosuppression had a spectacular debut with the finding, by Schwartz et al,,$^{31} 193194$ that immunological tolerance could be produced by the administration of purine-6-thiol (6-MP) with, or soon after, a dose of bovine gammaglobulin to rabbits. The production of tolerance was closely dependent on timing of drug and antigen administration, as shown in fig 1 . It also

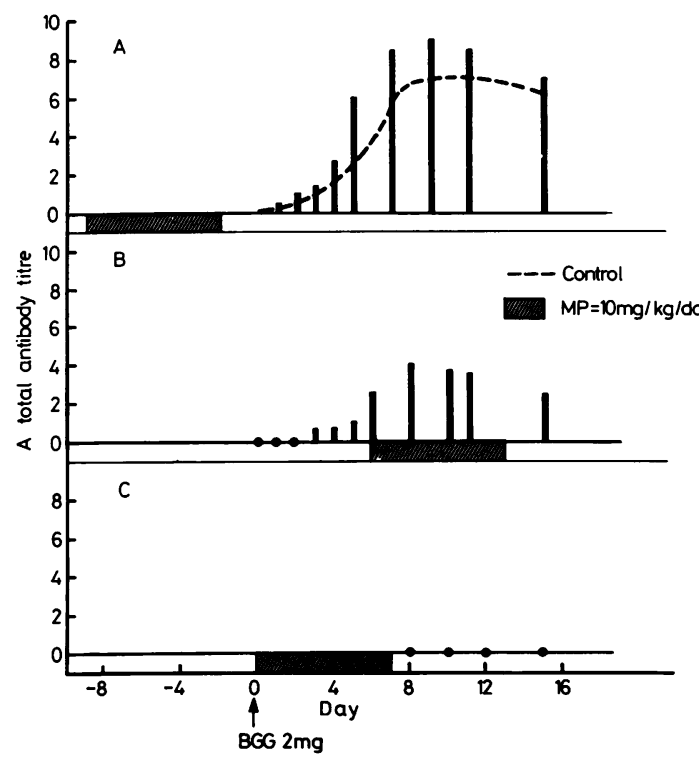

FIG 1 The effect of timing of drug administration with respect to antigenic stimulus. Data drawn from Schwartz et al. ${ }^{31} 193194$ In each case bovine gammaglobulin $2 \mathrm{mg} / \mathrm{rabbit}$ was administered on day 0 . In panel $A$ the drug (horizontal bar) was given before the antigen, in panel $B$ during antibody production, and in panel $C$ starting with antigen administration and continuing 7 days. was dependent on both the dose of antigen and the dose of drug, so that it had characteristics of the immune paralysis induced by massive doses of antigen. ${ }^{91}$ Moreover, both Schwartz et al and Frisch and Davies $^{73}$ showed that it could be highly specific. Frisch and Davies, for example, gave human erythrocytes to mice, waited two days, then gave a single dose of thioguanine and sheep red blood cells. Antibodies were produced to the sheep erythrocytes, but not to the human.

The observations of Schwartz and Dameshek ${ }^{194}$ were quickly developed in two directions. They began investigations of autoimmune diseases that are still continuing and are the major focus of today's workshop. The finding also provided entry into the field of organ transplantation, then awaiting only a means of controlling the rejection of allografted organs. This field has made rapid progress; today renal allografting is regarded not as an experimental, but as a therapeutic procedure, with a high probability of long-term survival. ${ }^{58}$ Almost from its inception, renal transplantation has involved the use of azathioprine, which had been synthesised originally as a masked form of 6-MP for anti-cancer studies, ${ }^{59}$ and it has superseded 6-MP, not only in this, but also in the autoimmune field. ${ }^{6092}$ An overview of the scope of this field is provided in table 1 which simply lists the types and numbers of clinical investigations known to be in progress in the USA.

Many of the problems and difficulties of interpretation that could be perceived in $1967^{91}$ are still with

TABLE 1 The types and numbers of clinical investigations of autoimmune disorders known to be in progress in the USA, using azathioprine

\begin{tabular}{llc}
\hline Category & $\begin{array}{l}\text { Number } \\
\text { of trials }\end{array}$ & $\begin{array}{l}\text { Total number } \\
\text { of patients }\end{array}$ \\
\hline Rheumatoid arthritis & 12 & 260 \\
Hepatitis & 2 & 265 \\
Reiter's & 1 & 10 \\
Graft vs host & $1(+1)$ & 50 \\
Multiple sclerosis & 2 & 200 \\
Crohn's & 8 & 400 \\
Myocarditis & 1 & 10 \\
Myasthenia gravis & $?$ & $?$ \\
Lupus (erythematosus) nephritis & $?$ & $?$ \\
\hline
\end{tabular}


us. However, striking progress has been made on several fronts and new ground is being broken on others. Before attempting to discuss these fields, it is probably pertinent to mention the numerous additional substances that have immunosuppressive activities. Most of these are still being drawn from the armamentarium of anti-tumour agents ${ }^{91}$ (table 2). It was this multiplicity of agents that prompted the question "are all immunosuppressants the same?" The short answer, of course, is "no". An attempt will be made in this paper to analyse the differences from several viewpoints and to point the way towards firmer knowledge in several essential, but possibly ancillary, areas.

The last two decades have witnessed remarkable advances in the recognition of lymphocyte types and functions. What in the early 1960's was simply a distinction between cell-borne and humoral immunity soon became a distinction between $\mathrm{T}$ and $\mathrm{B}$ lymphocytes. The importance of the thymus was elegantly shown by the experiment depicted in fig 2 . Meanwhile, the past decade has seen a rapid proliferation in the recognition of various subclasses of $T$ lymphocytes ${ }^{86110}$ and factors involved in their differentiation and activities. Much of this material will be dealt with elsewhere in this workshop; however, an attempt will be made here to present some of the effects of drugs on specific cell types. A selection among such reports is presented in table 3 . This is, in fact, a selection not guaranteed for immutability, since surveys of the literature reveal a dependence of such identifications on the specific conditions of the reporting laboratory. However, one must regard such identifications as a step in the understanding of the mechanisms of immunosuppression that may lead to the selective use of particular agents.

What may eventually be regarded as the most important breakthrough of all is the recent discovery that deficiencies in certain enzymes, concerned with purine intermediary metabolism, are associated with immunological defects. The first of these to be reported was congenital deficiency of adenosine deaminase (ADA) in a patient with severe combined immunodeficiency affecting both $\mathrm{B}$ and $\mathrm{T}$ cells. ${ }^{7 \mathrm{x}}$ Three years later, the same group found another

ІаВL.F 2 Immunosuppressive agents

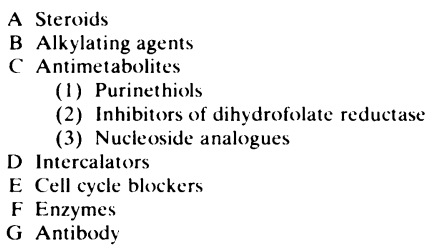

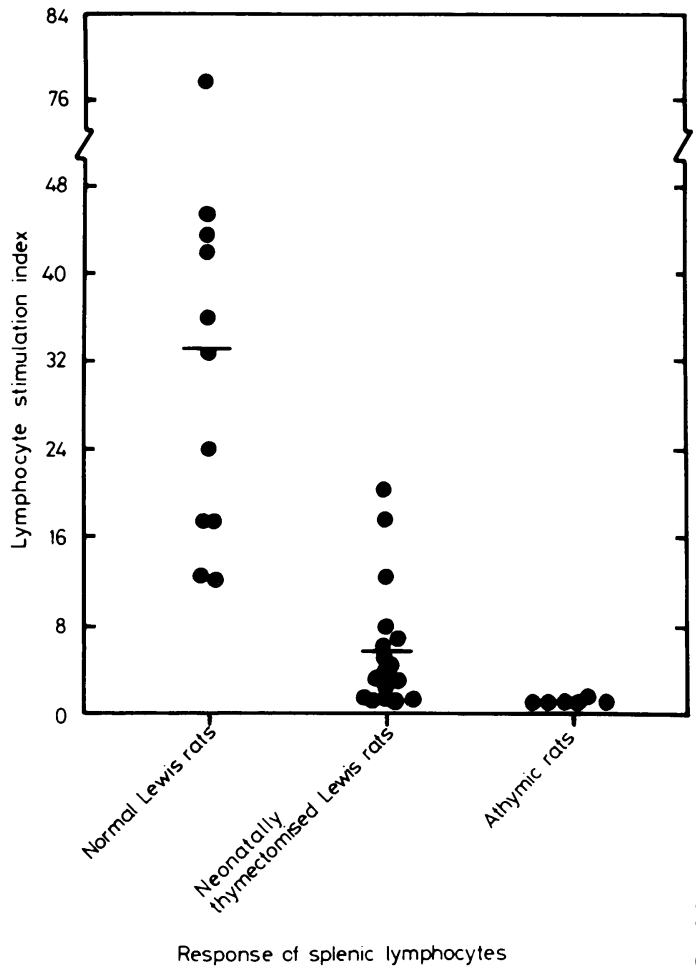

FIG 2 Documentation of the role of the thymus in the differentiation of lymphocytes. The responses of splenic lymphocytes to culturing in the presence of concanavalin $A$ are shown. (Courtesy of Life Sciences Research Reports 1981; 11: (1). SRI international Menlo Park, CA 94025, USA.)

TABLE 3 Reports of cellular responses to immunosuppressive agents

\begin{tabular}{lll}
\hline Agent & Effects & Reference \\
\hline Cyclosporin & TCGF response $\downarrow$ & 120 \\
Dexamethasone & TCGF production $\downarrow$ & 120 \\
Azathioprine & Inhibits RFC (T cells) & 53228 \\
Cyclophosphamide & $\downarrow$ B cells, $\downarrow$ suppressor T cells & 128197 \\
Melphalan & $\downarrow$ Activated B cells & 76 \\
BCNU & $\downarrow$ Activated and resting B cells & 76 \\
\hline
\end{tabular}

$\downarrow=$ decrease.

TCGF $=\mathrm{T}$ cell growth factor

$\mathrm{RFC}=$ rosette forming cell.

$\mathrm{BCNU}=$ carmust in

deficiency, of purine nucleoside phosphorylase (PNP), ${ }^{77}$ that may eventually be even more helpful in understanding the biochemical events of the immune response. Most of the explanations of the effects of ADA deficiency revolve around the toxicity of deoxyadenosine. However, PNP deficiency is selectively toxic to $\mathrm{T}$ cells. ${ }^{77}$ Deficiencies in phosphoribosyltransferases have only minor effects, although some impairment of B cell activities have 
been noted. ${ }^{3}$ The explanation of the selective effects of PNP deficiency is attributable to the existence of a deoxycytidine kinase that phosphorylates d-guanosine (d-Guo), whereas in the ribo-series there is no similar enzyme. The deoxyguanylate eventually reaches the triphosphate stage and exerts a feedback inhibition of ribonucleotide reductase. The phosphorylation is the preferred route of metabolism of d-Guo in T cells, but is almost nonexistent in B cells. Thus, d-Guo is highly toxic to T lymphoblastoid cell (T-LCL) lines but only mildly so to B-LCL, and not at all to B-PNP deficient. ${ }^{163}$ These findings are summed up in table 4 .

It is apparent that observations of this sort may not only lead to more precise definitions of immune response, but lay the basis for rational approaches to selective drugs.
TABLE 4 Enzyme deficiencies affecting immune responses

\begin{tabular}{|c|c|c|}
\hline Deficiency & $\begin{array}{l}\text { Lymphocytes } \\
\text { inhibited }\end{array}$ & $\begin{array}{l}\text { Biochemical } \\
\text { abnormalities }\end{array}$ \\
\hline $\mathrm{ADA}$ & $\begin{array}{l}\text { B } \\
\text { T }\end{array}$ & $\begin{array}{l}\text { d-Ado excretion } \\
\uparrow d \text { ATP, ATP, cAMP }\end{array}$ \\
\hline PNP & $\mathrm{T}$ & $\begin{array}{l}\uparrow d \text {-Guo } \\
\uparrow d \text {-GTP } \\
\downarrow_{\text {nucleotide reductase }}\end{array}$ \\
\hline APRT & none & 2. 8 -diOH-6- $\mathrm{NH}_{2}$ purine $\rightarrow$ \\
\hline HGPRT & none & †uric acid \\
\hline
\end{tabular}

Above the line, abnormalities producing major effects on immune responses. Below the line, deficiencies having at most minor effects. $A D A=$ adenosine deaminase, $\mathrm{PNP}=$ purine nucleoside phosphorylase, $\mathrm{APRT}=$ adenine phosphoribosyl transferase, HGPRT = hypoxanthine guanine phosphoribosyl transferase, $\mathrm{d}-\mathrm{Ado}=$ deoxyadenosine, $\mathrm{ATP}=$ adenosine triphosphate, $\mathrm{dATP}=$ the corresponding deoxyribose derivative, $\mathrm{cAMP}=$ cyclic adenylate, $\mathrm{d}-\mathrm{Guo}=$ deoxyguanosine, $\mathrm{d}-\mathrm{GTP}=$ deoxyguanosine triphosphate. Arrows signify increases (up) and decreases (down). 


\title{
The comparative incidence of malignant disease in rheumatoid arthritics exposed to different treatment regimens
}

\author{
B R I A N L HAZLEMAN \\ From the Department of Rheumatology, Addenbrooke's Hospital, Hills Road, Cambridge \\ PRESENTED B Y M DE SILVA \\ Senior Registrar, Addenbrooke's Hospital, Cambridge
}

SUMmary Cytotoxic drugs are effective in disease suppression and affect the immune system. The correlation between these is poor, in many patients, at the dosage used in man. This may partly explain why the large increased risk of neoplasia, initially predicted, has not been shown to follow the use of cytotoxic therapy.

The cause of death in patients with rheumatoid arthritis has been assessed both by surveys based on clinical populations and by reviews of necropsy records. There have been few attempts to assess the influence of drug therapy on mortality, or on the incidence of neoplasia. In addition, evidence is accumulating to suggest that lymphoreticular tumours may be associated with autoimmune disease.

There have now been several long-term studies of patients with rheumatoid arthritis treated with immunosuppressives; azathioprine and cyclophosphamide in particular. These studies are reviewed and it is concluded that the incidence of most of the common cancers is not increased.

The use of immunosuppressive drugs in rheumatoid arthritis has, in general, proved to be of benefit. ${ }^{82} 143$ Azathioprine or cyclophosphamide, started early in the course of rheumatoid arthritis, have been shown to be equally effective and similar in their efficacy to gold therapy. ${ }^{38}$ Since a short course of such treatment does not produce a lasting remission, long-term administration would be expected in many instances. This focuses attention on the adverse effects of prolonged administration of these drugs including bone marrow suppression, susceptibility to infection and the potential development of malignant tumours. Some of the potential toxic side effects are listed in table 1 .
TABLE 1 Toxicity of immunosuppressive drugs

\begin{tabular}{|c|c|c|c|c|}
\hline & $\begin{array}{l}\text { Cyclophos- } \\
\text { phamide }\end{array}$ & Azathioprine & $\begin{array}{l}\text { Chloram- } \\
\text { bucil }\end{array}$ & Methotrexate \\
\hline $\begin{array}{l}\text { Gastrointestinal } \\
\text { intolerance }\end{array}$ & + & + & + & + \\
\hline Infection & + & + & + & + \\
\hline Cystitis & ++ & - & - & - \\
\hline Hair loss & ++ & - & + & + \\
\hline Hepatic damage & - & + & - & ++ \\
\hline Leucopenia & t+ & + & ++ & + \\
\hline Azoospermia & ++ & - & $+t$ & + \\
\hline Anovulation & ++ & - & ++ & - \\
\hline Teratogenesis & + & $+?$ & + & + \\
\hline Neoplasia & + & + & + & ? \\
\hline
\end{tabular}

The relative contributions to the patient's increased susceptibility to infections arising from the disease itself, and from the frequent concomitant steroid therapy, remain difficult to evaluate. Infections with organisms such as cytomegalovirus, herpes simplex, mycobacterium tuberculosis and fungi are possible. The use of cyclophosphamide may be accompanied by alopecia, haemorrhagic cystitis and bladder fibrosis; it may also lead to ovarian failure and azoospermia. Azathioprine is apparently free of such side effects.

Cytostatic drugs are potentially teratogenic in man, although several women receiving azathioprine have conceived and delivered healthy babies. Methotrexate, if given repeatedly, can lead to hepatic fibrosis. The most obvious effect of these drugs is on the haemopoietic system. Since they affect rapidly proliferating cells they have an effect on bone marrow precursors, such as stem cells and the myeloid and erythroid, as well as the lymphoid, series.

\section{Induction of malignant disorders}

The most difficult general problem to assess, associated with the use of immunosuppressive agents, is the potential threat of the development of neoplasia. It is 
theoretically possible that the development of a neoplasm may occur many years after continuous immunosuppressive therapy or even after discontinuation of such therapy. It took many years to recognise the increased risk of leukaemia that occurs in patients who had received radiation therapy for ankylosing spondylitis some 20 to 25 years previously. ${ }^{37}$

Over the last decade considerable evidence has accumulated suggesting that immunosuppressive and cytotoxic drugs cause an increased incidence of malignancy, particularly when used for long periods. Most of the evidence is derived from studies of patients receiving organ transplants, and more anecdotal evidence comes from the use of these drugs in rheumatic diseases. Animal experiments show that immunosuppression facilitates the transplantation of malignant cells, increases the incidence of virus-induced cancers and accelerates the growth of metastases. Perhaps the expected increased risk of neoplasia, initially predicted, has not materialised because there is little correlation between efficacy of disease suppression and effect on the immune system in man.

Recently a prospective study ${ }^{115}$ of 3823 patients who had received a renal transplant and of 1349 other patients, including cases of rheumatoid arthritis, receiving cytotoxic drugs (particularly azathioprine), showed no clear evidence that immunosuppressive drugs produce an increased risk of most of the common cancers. However, few of the patients had been under observation for over five years, and an increase may appear later. Follow-up of the renal transplant recipients showed an almost 60 -fold increase of non-Hodgkin's lymphoma together with an excess of squamous cell skin cancer and mesenchymal tumours. The patients without transplants showed an excess of the same tumours, though to a lesser extent.

The most striking excess of lymphoid tumours occurred as non-Hodgkin's lymphomas (mainly described as reticulum cell sarcomas and microgliomas) in the transplant series, which confirms what has been reported before. ${ }^{94}$ This excess of lymphoid tumours was extraordinary in its magnitude, predilection for the brain and very short induction period. Possible hypotheses to explain these findings include disturbance of immunosurveillance or of graft-versus-host reaction, chronic antigenic stimulation and the effect on oncogenic viruses. For each of these there is laboratory support. The short induction period, sometimes within a few months after transplantation, suggests a viral origin, since transformation could take place immediately if the virus was already present.

That patients without transplants also showed an excess of lymphoid tumours suggests that immunosuppression is important, though the presence of a graft may contribute to tumour development, since in transplant recipients the excess was much greater. Other observations support the primary importance of immunosuppression. Firstly, there was no tendency for lymphoid tumours to be more common in recipients who experienced repeated rejection crises, nor in those who had had multiple grafts. Secondly, a study of patients treated by renal dialysis, who have severely depressed immune reactions, showed that they also had an increased incidence of non-Hodgkin's lymphoma, and that in one of them, who had never been given immunosuppressive drugs, the lymphoma affected the brain. Thirdly, certain rare hereditary disorders characterised by major immunological impairment, such as the Wiskott-Aldrich syndrome, are associated with an increased risk of lymphomas.

A direct mutagenic effect of the chemical agents appears to be unlikely because of the abrupt increase in incidence, the subsequent constancy of the risk from the time of first exposure and the lack of any noticeable dose effect, all of which are in pronounced contrast to the usual findings with known chemical carcinogens.

Only four patients without transplants developed non-Hodgkin's lymphoma (three of whom were treated with azathioprine). ${ }^{115} \mathrm{~A}$ three-fold excess of lymphomas was observed in a large series of patients with rheumatoid arthritis in Finland ${ }^{101}$ and it was not clear whether this was associated with any particular form of treatment. However, cases of lymphoma affecting the brain in patients without transplants, but receiving immunosuppressive drugs, have been reported. ${ }^{127} 220221$

The risk of non-Hodgkin's lymphoma in the study of Kinlen et $a l^{115}$ was smaller in patients without transplants than in transplant recipients. Differences in the type, amount and duration of drug treatment were, however, substantial. Azathioprine was given initially only to $64 \%$ of patients in the non-transplant series and was continued for over two years in less than $30 \%$, whereas azathioprine was continued in over $90 \%$ of the transplant recipients throughout the period of observation. Similar differences were observed in the case of prednisone.

Walder et al in $1971^{231}$ emphasised the common occurrence of squamous carcinomata in transplant recipients. Kinlen et $a l^{115}$ also reported a ten-fold increase in mortality from skin cancer in transplant recipients. In the latter study there were three cases of acute leukaemia, all in transplant recipients, compared with the expected figure of less than one, and six mesenchymal tumours when none was expected; three of these were in the non-transplant group. There were four cases of bladder cancer in patients 
without transplants and the risk of this disease is related specifically to the use of cyclophosphamide.

In order to try to distinguish between the effects of immunosuppressive therapy and the disease process in rheumatoid arthritis, surveys of the cause of death must be examined.

\section{Cause of death}

The cause of death in patients with rheumatoid arthritis has been assessed both by surveys based on clinic populations and by reviews of necropsy records. ${ }^{1335575102151156167219226}$ There have been few attempts to assess the influence of drug therapy on mortality or on the incidence of neoplasia.

Most surveys of the causes of death in rheumatoid arthritis agree that patients with the disease have a higher mortality rate than the rest of the population, though they die not so much from specific complications of the disease as from all the usual causes of death, but at an earlier age than usual.

In 1970 Uddin et $a^{219}$ reviewed 475 patients with rheumatoid arthritis, first seen between 1954 and 1966. The cumulative survival rate was determined in each year for up to ten years, compared with that of a normal matched population. This was lower than expected for each sex at each year of follow-up, particularly in the later years.

Cobb et al in $1953^{33}$ followed 583 patients for a mean duration of $9 \frac{1}{2}$ years. The overall mortality rate was 24.4 per 1000 patients per year in contrast to 18.9 for a comparable group matched for age and sex. More recently, Isomaki et al in $1975^{102}$ observed 1000 patients with rheumatoid arthritis for three years. All were over 40 years of age. Deaths were compared with an age and sex matched control population. The 122 deaths in the rheumatoid group compared with 69 in the controls. Deaths from cancer were fewer in the rheumatoid group.

It is difficult to compare studies of the causes of death in rheumatoid patients. Most studies rely on certified causes of death which, on occasion, do not correlate well with findings at necropsy. There are also differences in the periods of follow-up.

The improved hospital supervision of rheumatoid patients and the use of an expanded range of therapeutic agents in the last decade may invalidate comparisons with early studies.

Reports of the incidence of neoplasia, independent of therapy, vary between studies. Only Moesman in $1969^{151}$ found a greater frequency of malignant disease in a small group of elderly patients.

\section{Induction of malignant disorders}

In view of reports of malignancy, Hunter et al in
$1975^{95}$ carried out chromosomal studies in patients receiving long-term azathioprine. This showed a two-fold increase in chromosomal abnormalities in patients, similar to that reported during cyclophosphamide treatment of rheumatoid arthritis by Tolchin et al in $1974 .{ }^{216}$ Since similar changes may occur with phenylbutazone, and a variety of other drugs, its significance will have to await long-term observation.

Evidence is accumulating to suggest that lymphoreticular tumours may be associated with autoimmune disease. ${ }^{6 \times 149211}$ Patients with Sjögren's syndrome are especially prone to develop extensive lymphocyte infiltrates in extrasalivary organs with pseudolymphoma formation and occasional progression to malignant lymphoma. ${ }^{6}$ Three series of patients with systemic lupus erythematosus have shown an increased risk of cancer. ${ }^{29} 52126$

Azathioprine has been widely used as an immunosuppressive agent in the United Kingdom, whereas cyclophosphamide is used more widely in North America.

Owing to the influence of de Seze and Kahn, ${ }^{46}$ chlorambucil has been the most widely used immunosuppressive drug in France. This choice was based on the use of the drug in chronic lymphatic leukaemia where there were hopes that it would have a greater affinity for lymphocytes. The enthusiasm for the drug declined during the 1970's on account of its side effects. Deshayes $e t$ al $^{47}$ summarised the results concerning side effects of chlorambucil noted by several French authors in a total of 495 patients, and compared these to the effects of azathioprine and cyclophosphamide.

Treatment linked mortality probably depends on the duration of exposure to risk, which is difficult to determine for a treatment with prolonged aftereffects. Its assessment depends on the length of time for which the patients are followed after treatment has begun, and on whether all deaths are included or only those which can be attributed to the treatment. Most important is the fact that no true control group exists, and a comparison can only be made with the life expectancy of the general population of the same age or that of a random group of rheumatoid patients.

Farber et $\mathrm{l}^{64}$ compared 126 patients treated with cytotoxic drugs with 126 age and sex matched rheumatoid arthritic patients in hospital during the same years but who did not receive cytotoxic therapy. Patients predominantly received nitrogen mustard or cyclophosphamide or both. The patients were followed from 1965 to 1974 and no increase in malignancy was recorded, nor were any lymphomas seen. This view is not shared by others. Parsons and co-workers in $1974^{169}$ carried out a long-term follow-up of patients with rheumatoid arthritis treated with cyclophosphamide. They found eight 
malignancies including seven of the lymphoproliferative variety, but the incidence of Sjögren's syndrome is not recorded. Pollock et al in $1973^{179}$ reported two malignancies in rheumatoid arthritic patients treated with cyclophosphamide (both solid tumours), and a recurrence of a previously treated melanoma was reported in a patient following cyclophosphamide therapy. ${ }^{138}$

Renier et al in $1978^{1 \times 4}$ followed 131 cases of rheumatoid arthritis for at least four years and recorded 13 deaths through malignant disease, of which seven were haemopoietic neoplasms. Cancer was diagnosed in five other patients. Eighteen of the 131 patients had received chlorambucil, and then later cyclophosphamide, and seven of the cases of malignant disorder were observed in these 18 patients.

Four cases of leukaemia have arisen in association with rheumatoid arthritis following treatment with azathioprine,,$^{32131196}$ and in two cases following treatment with cyclophosphamide. ${ }^{131}$

Kahn et al in $1979,{ }^{108}$ using results from a collective retrospective study, calculated the incidence of acute leukaemia during and after cytotoxic therapy. The results of this study, which included 2006 patients whose average age was 55 years, are shown in table 2 . A total of 1711 patients had received chlorambucil and were followed for one to 13 years. One third of the patients were not followed up. Kahn et al ${ }^{108} \mathrm{com}$ pared these figures with cyclophosphamide treatment (three cases of leukaemia in a co-operative study of 229 patients) and with azathioprine, where Seidenfeld et al $^{196}$ noted an incidence of $1 \cdot 3 \%$. Kahn et al emphasised that no case of leukaemia was seen in patients who received less than $1 \mathrm{~g}$ chlorambucil and in whom the duration of treatment was less than six months. The nature of the acute leukaemia with chlorambucil was variable, and the delay from the beginning of treatment to the appearance of leukaemia was $5 \cdot 7 \pm 2.9$ years. ${ }^{108} 145$ The risk of developing leukaemia seems more pronounced in children suffering from chronic rheumatoid arthritis; three cases out of 40 infants treated with chlorambucil compared with 0 out of 160 control infants. ${ }^{23}$

TABLE 2 Results of a survey of the incidence of acute leukaemia during and after therapy with chlorambucil (Kahn et $\left.a l^{108}\right)$

\begin{tabular}{lcc}
\hline Reason for therapy & Number in study & $\begin{array}{l}\text { Number of cases } \\
\text { of leukaemia }\end{array}$ \\
\hline Rheumatoid arthritis & 1612 & 12 \\
Systemic lupus erythematosus & 44 & 0 \\
Psoriatic arthritis & 35 & 4 \\
Others & 20 & 0 \\
Total & 1711 & 16 \\
\hline
\end{tabular}

\section{Study of cause of death with particular reference to azathioprine}

In view of the few attempts to assess the influence of drug therapy on the incidence of neoplasia, the author initiated a survey on a group of closely followed patients with rheumatoid arthritis predominantly treated with azathioprine. ${ }^{125}$ The patients in this study were unusual in that treatment with azathioprine was started, in some patients, as long as 11 years previously, often in high dosage (5 $\mathrm{mg} / \mathrm{kg} /$ day) and sodium aurothiomalate (Myocrisin) had been given in combination. For purposes of comparison, an analysis of mortality was performed by calculation of expected mortality in the patient group, based on rates for a similar age and sex distributed sample of the general population.

A total of 311 patients with classical or definite rheumatoid arthritis was studied over this 11-year period. Follow-up was complete in all patients. The death certificate was obtained for all the 46 who died, and results of necropsy were available in 19 cases.

A total of 214 patients received cytotoxic therapy (table 3 ) and 63 received cyclophosphamide (range $0 \cdot 2$ to 8 years) or chlorambucil, alone or in combination with azathioprine, at some stage in their disease. The mean duration of treatment in the 151 patients treated with azathioprine alone was $2 \cdot 5$ (range 1 to 11) years, a total of 500 patient years.

The tumours diagnosed in both the dead and the survivors are recorded in table 4 . Five patients have had apparently successful treatment of their neoplasm (three patients had received cytotoxic therapy before the discovery of the neoplasia). Ten of the 20 neoplasms occurred in patients who were receiving cytotoxic therapy (table 5). There is no evidence to link rheumatoid arthritis with any particular form of tumour.

The one case of lymphosarcoma occurred in a 71-year-old patient, without evidence of Sjögren's syndrome, who had not received cytotoxic therapy.

TABLE 3 Malignancy associated with treatment

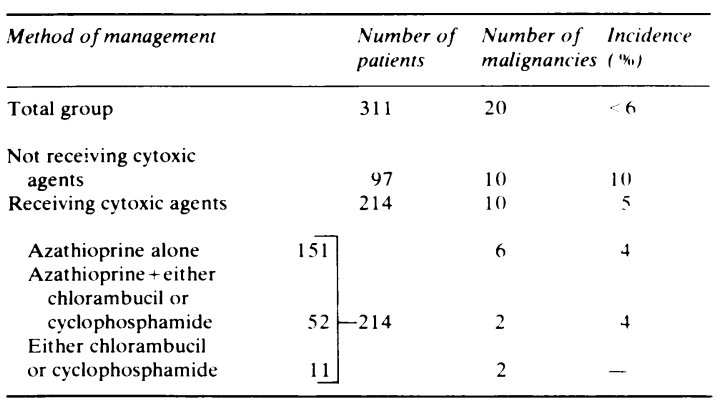


TABLE 4 Neoplasia occurring in 311 patients with rheumatoid arthritis (1966-1976)

\begin{tabular}{lc}
\hline Type of neoplasm & \\
\hline Lung/bronchus & 5 \\
Breast & 4 \\
Prostate & 2 \\
Uterus & 1 \\
Rectum & 1 \\
Colon & 1 \\
Bowel unspecified & 1 \\
Thyroid (anaplastic) & 1 \\
Multiple bladder neoplasia & 1 \\
Unspecified & 1 \\
Lymphosarcoma & 1 \\
Basal cell carcinoma of skin & 1 \\
Total & 20 \\
\hline
\end{tabular}

TABLE 5 Neoplasia in group treated with cytoxic drugs

\begin{tabular}{lll}
\hline & Therapy & Duration (years) \\
\hline Carcinomas-deceased patients & & \\
Lung/bronchus & Cyclophosphamide & $2 \cdot 3$ \\
& Azathioprine & $0 \cdot 3$ \\
Lung/bronchus & Azathioprine & 5 \\
Lung/bronchus & Chlorambucil & $1 \cdot 7$ \\
& Azathioprine & 3 \\
Breast & Intra-articular & \\
cyclophosphamide & - \\
Rectum & Azathioprine & 2 \\
Unspecified & Azathioprine & $1 \cdot 5$ \\
Carcinomas-prevalent & Azathioprine & 8 \\
Uterus & Azathioprine & 3 \\
Thyroid & Cyclophosphamide & 8 \\
Multiple bladder neoplasm & Azathioprine & 5 \\
Basal cell carcinoma & & \\
\hline
\end{tabular}

The principal causes of death are listed in table 6 . Ischaemic heart disease heads the list, followed by neoplasia. Deaths from infections all involved the lung and the incidence is lower than previously recorded in patients with rheumatoid arthritis. ${ }^{24}{ }_{167}$

The observed death rate among the rheumatoid arthritis patients, from all causes according to age, has been compared with expected values obtained from the Registrar General's records using a $\chi^{2}$ test. The death rate was higher than expected in the 45 to 64-year-old age group, but lower in those aged 75 years or over. The differences are significant $(p<0.0005)$. Analysis of age related specific causes of death (table 7) showed an increased frequency of deaths from neoplasia and ischaemic heart disease occurring in the 45 to 64 -year-old age group, but this is not significant.

There was increased evidence of neoplasia in the patients with rheumatoid arthritis (table 3 ) but this was not associated with immunosuppressive treatment, nor was there any difference in duration of treatment between those developing tumours compared with the rest.

One patient developed a transitional cell carcinoma of the bladder after eight years' therapy with cyclophosphamide. This is of interest, as Wall and
TABLE 6 Causes of death in rheumatoid arthritis (46 patients)

\begin{tabular}{lrl}
\hline Cardiovascular & & \\
Ischaemic heart disease & 15 & $(7)^{*}$ \\
Cor pulmonale & 2 & $(1)$ \\
$\quad$ Pulmonary embolus & 2 & $(1)$ \\
Neoplasia & 13 & $(2)$ \\
Respiratory infections & 6 & $(3)$ \\
Chronic pulmonary disease & 3 & $(2)$ \\
Others & & $(2)$ \\
$\quad$ Adrenal failure & 2 & \\
Cirrhosis; portal hypertension & 1 & $(1)$ \\
Peptic ulceration & 1 & \\
Cervical subluxation & 1 & \\
\hline
\end{tabular}

${ }^{*}$ Number in parentheses indicates confirmed at necropsy.

TABLE 7 Rheumatoid arthritis: age related specific causes of death (1966-1976)

\begin{tabular}{llllll}
\hline $\begin{array}{l}\text { Age } \\
(y r)\end{array}$ & \multicolumn{2}{c}{ Neoplasia } & & \multicolumn{2}{c}{ Ischaemic heart disease } \\
\cline { 2 - 3 } \cline { 5 - 6 } \cline { 5 - 6 } & Observed & Expected & & Observed & Expected \\
\hline $25-44$ & - & 0.1 & & - & 0.320 \\
$45-64$ & 6 & $3 \cdot 3$ & & 10 & $2 \cdot 626$ \\
$65-74$ & 5 & $4 \cdot 6$ & & 5 & $5 \cdot 117$ \\
$75-$ & 2 & $1 \cdot 2$ & & - & $2 \cdot 521$ \\
Totals & 13 & 9.4 & 15 & 10.584 \\
\hline
\end{tabular}

Clausen in $1975^{232}$ described five cases of carcinoma of the bladder after prolonged cyclophosphamide therapy. In each case interstitial haemorrhagic cystitis preceded the development of the carcinoma. The patients all developed fatal and invasive carcinomas. Dale and Smith in $1974^{39}$ suggested that the tumour could be small and possibly non-recurrent. The patient in this study has had local recurrences which have been removed at routine cystoscopy.

The presence of haemorrhagic cystitis in a patient receiving cyclophosphamide should suggest immediate and permanent discontinuation of the drug. However, the cessation of haematuria and withdrawal of the drug does not eliminate the need for cystoscopy, and probably this should be repeated periodically, indefinitely thereafter. Previous observations have shown atypical epithelial cells on cytological examination of urine in haemorrhagic cystitis during treatment with cyclophosphamide. ${ }^{68} \mathrm{~A}$ necropsy study showed that ten of 49 patients who had received long-term cyclophosphamide had signs of urinary bladder fibrosis, the frequency being related to dose and duration of therapy. In about $50 \%$ of cases the fibrosis did not produce symptoms.

These findings suggest caution in the use of cyclophosphamide for non-malignant conditions. It is suggested that protracted periods of treatment should be avoided, and that the drug be used in intermittent courses if at all possible.

Louie and Schwart $\mathrm{z}^{130}$ compiled a list of 109 treated patients who developed neoplasms and compared them with patients who did not receive 
immunosuppressive therapy. The most prominent difference between the treated and untreated patients was the increased incidence of acute myeloblastic leukaemia in the treated group. The most important association was therapy with an alkylating agent. Certainly this review of the literature suggests that these agents are more likely to produce problems. However, assessment of the true risk is difficult as we do not know the expected number of neoplasms in untreated patients.

The potential oncogenic effect of immunosuppressive drugs remains one of the main deterrents to their wide-spread use in the treatment of rheumatoid arthritis. Further studies are required to assess the true influence of these drugs on the incidence of tumours in rheumatoid arthritis. 


\title{
Long-term effects of azathioprine in rheumatoid arthritis*
}

\author{
M B UROWITZ, H A SMYTHE. T ABLE, C S NORMAN AND C TRAVIS \\ From the Rheumatic Disease Unit, Wellesley Hospital, 160 Wellesley Street E, Toronto, Ontario, Canada \\ M4Y $1 J 3$.
}

SUMMARY Efficacy and safety of azathioprine in 'high' and 'low' dose regimens in rheumatoid arthritis (RA), both in short-term studies and in follow-up over 40 months, have previously been shown. In the present report, 36 patients with RA treated with azathioprine (group I) and 49 age-matched patients with RA (group II), were studied to detect potential early markers of malignancy.

Chest $x$-rays were similar in both groups. One patient in group I had a positive PAP smear and was subsequently found to have uterine carcinoma. Alpha-fetoprotein was positive in one patient in group I and none in group II. CEA was negative in all patients in group I, but positive in seven in group II. On chromosomal analysis group I showed a greater frequency of breakage. Group I showed lower serum folates and a highly significant number of megaloblastic features in marrow aspirates.

In group I seven tumours, three being malignant, occurred while taking azathioprine, and in group II six tumours, one malignant, were identified $(\mathrm{p}=0 \cdot 17)$.

The apparent increased risk of malignancy previously suggested by others warrants further studies with larger populations and over a continuous longer period.

Azathioprine has been shown to be an effective agent in the treatment of active rheumatoid arthritis (RA). ${ }^{38124143224}$ The long-term side-effects of this purine analogue, especially the potential for the induction of neoplastic change, have been described only sporadically in patients with RA. In 1976, two patients with seropositive RA, treated with both azathioprine and alkylating agents and who developed leukaemia, were reported from this unit. ${ }^{196}$ This prompted us to examine a group of patients with RA on azathioprine, with respect to the incidence of neoplasms, to try to detect any specific

-This work was supported by the Canadian Arthritis Society, 920 Yonge Street, Ste 420, Toronto, Ontario, Canada. Grant Number: 7-195-74. clinical or laboratory markers in patients with neoplastic change.

\section{Method}

Thirty-six patients with classical or definite RA by American Rheumatism Association (ARA) criteria ${ }^{186}$ receiving azathioprine were chosen to take part in this in-patient study.

Forty-nine age and sex matched patients, with classic or definite RA, who volunteered for a 'cance check-up', were chosen from our in-patient and out patient population as controls. These patients were? on standard anti-inflammatory and disease suppressant (gold, chloroquine, penicillamine) medications; none had ever taken azathioprine or any cytotoxic agents. A medical history was obtained from each patient and a complete physical examination was performed. Total active joint count, grip strength ${ }^{191}$ and functional class were recorded.

Laboratory tests performed included: haemoglobin, white blood cell (WBC) count and differential, platelet count, erythrocyte sedimentation rate (ESR) by the Westergren method, latex fixation test, ${ }^{201}$ SGOT, alkaline phosphatase, protein electrophoresis, serum iron and total iron binding capacity. Serum folate determinations before July 1977 were performed using a microbiological assay. ${ }^{36204}$ After July 1977, a radiodilution method was adopted (Amersham Folate Radioassay Kit). Serum $\mathbf{B}_{12}$ was performed by a radiodilution method. ${ }^{183}$ Alpha-fetoprotein was measured by immunodiffusion in agar gel. ${ }^{164}$ Carcino-embryonic antigen (CEA) was detected by radioimmunoassay (Hoffman-LaRoche Kit). Urine and sputum cytology, when obtainable, was studied on each patient.

Radiological examinations included chest $x$-rays on all patients, mammography on each female and barium contrast studies, when indicated. All patients were examined by a dermatologist with attention to neoplastic changes of the skin. All women were 


\section{Long-term effects of azathioprine in rheumatoid arthritis}

Suppl p 19

examined by a gynaecologist and cervical and vaginal smears were taken.

Bone marrow aspiration was performed on all patients receiving azathioprine and the samples examined by one of us (CSN). Marrow chromosomal analyses ${ }^{153} 195215$ were performed on 25 patients of the 36 in the azathioprine group and in 11 of the 49 controls.

Statistical analyses of the results were carried out using the Fisher's exact test and the $\chi^{2}$ test. Significance testing was carried out at the $5 \%$ level.

\section{Results}

Group I (azathioprine treated) consisted of 36 patients, eight males and 28 females. Group II (control) consisted of 49 patients, 13 male and 36 female. There was no significant difference between the groups with respect to sex. The mean age was significantly higher in group I than in group II ( 57.8 years vs 51.4 years). Group I had a slightly longer disease duration ( 15.3 years vs 11.9 years) (table 1$)$.

Group I had taken azathioprine for a mean duration of 38.5 months. The mean dose of drug for the group at assessment was $1.08 \mathrm{mg} / \mathrm{kg} /$ day. This was less than the mean dose at initiation of azathioprine treatment $(1.33 \mathrm{mg} / \mathrm{kg} / \mathrm{day})$. When the groups were compared with respect to concomitant medications within the three months before assessment, more group I patients had taken chloroquine and for a longer duration than patients in group II. There was a higher frequency of gold and penicillamine treatment in the control group at the time of assessment. Eleven patients in group I were taking low-dose prednisone, while only two patients in group II were on prednisone. Four patients in the azathioprine treated group had had intra-articular injections of their knees with radioactive gold; three patients had had both knees injected since the diagnosis of RA. One patient in group II had had a knee injected with radioactive yttrium and one had had a knee injected with radioactive gold. All patients had had intra-articular steroids at some time in their course. None was taking phenylbutazone.

TABLE 1 Study population

\begin{tabular}{llll}
\hline & $\begin{array}{l}\text { Group I; } \\
\text { azathioprine } \\
\text { treated }\end{array}$ & $\begin{array}{l}\text { Group II; } \\
\text { control }\end{array}$ & Significance \\
\hline No of patients & 36 & 49 & \\
$\quad$ Male/female & $8 / 28$ & $13 / 36$ & \\
$\quad$ Age $(\mathrm{yr})$ & 57.8 & 51.4 & $\mathrm{p}=0.05$ \\
$\begin{array}{l}\text { Disease duration (yr) } \\
\text { Duration of }\end{array}$ & 15.3 & 11.9 & $\mathrm{p}=0.22$ \\
$\quad$ azathioprine (mth) & 38.5 & & \\
Dose of azathioprine & $1.08 \mathrm{mg} / \mathrm{kg} / \mathrm{day}$ & & \\
\hline
\end{tabular}

Age, duration and dose are given as means.
TABLE 2 Clinical features

\begin{tabular}{llll}
\hline & $\begin{array}{l}\text { Azathioprine } \\
\text { treated (36) }\end{array}$ & $\begin{array}{l}\text { Control } \\
(49)\end{array}$ & Significance \\
\hline Active joint count & $11 \cdot 3$ & $16 \cdot 2$ & $\mathrm{p}=0.02$ \\
$\begin{array}{l}\text { Grip strength }(\mathrm{mmHg}) \\
\text { ESR (mm/h) }\end{array}$ & 150 & 126 & $\mathrm{p}=0.12$ \\
$\begin{array}{l}\text { Extra-articular } \\
\text { features/patient }\end{array}$ & $51 \cdot 3$ & $23 \cdot 3$ & $\mathrm{p}=0.000002$ \\
\begin{tabular}{l} 
Latex titre* \\
\hline
\end{tabular} & $1 \cdot 1$ & 0.9 & \\
\hline
\end{tabular}

*Tube numbers: tube $1=$ titre $1 / 40$, thereafter doubling dilutions.

TABLE 3 Laboratory features: haematology

\begin{tabular}{llll}
\hline & $\begin{array}{l}\text { Azathioprine } \\
\text { treated (36) }\end{array}$ & $\begin{array}{l}\text { Control } \\
(49)\end{array}$ & Significance \\
\hline Haemoglobin $(\mathrm{g} / \mathrm{dl})$ & 12.76 & 12.77 & $\mathrm{p}=0.13$ \\
WBC $\left(\mathrm{mm}^{3}\right)$ & 5591 & 7311 & $\mathrm{p}=0.002$ \\
Polymorphs $\left(\mathrm{mm}^{3}\right)$ & 3931 & 5225 & $\mathrm{p}=0.23$ \\
Lymphocytes $\left(\mathrm{mm}^{3}\right)$ & 1181 & 1720 & $\mathrm{p}=0.15$ \\
Platelets $\left(\mathrm{mm}^{3}\right)$ & 287972 & 368827 & $\mathrm{p}=0.08$ \\
\hline
\end{tabular}

Clinically, both groups were comparable as to grip strength, extra-articular manifestations and latex titre (table 2). They differed significantly in the joint count, with group II having a greater number of active joints (16.2 vs $11 \cdot 3)$, and ESR, with group II having a lower ESR $(51 \cdot 3$ vs $23 \cdot 3)$. There were no marked differences in fertility, hormone treatment and history of cigarette smoking between the groups.

Both groups were comparable in terms of SGOT, alkaline phosphatase and protein electrophoresis results. The groups did not differ with respect to iron studies, haemoglobin and platelet, neutrophil and lymphocyte counts. The total white blood cell count was significantly lower in the azathioprine treated group (table 3 ).

Chest $x$-rays were similar in both groups. Inflammatory and fibrotic abnormalities were seen in 15 patients in group I and nine patients in group II. There were no suspicious lesions seen in either group. Mammography showed a comparable number of benign lesions, that is, fibrosis, generalised calcifications and/or dysplasia. No masses or signs of malignancy were seen except for one suspicious lesion in a woman in group I and three in group II. These women are being followed carefully and, thus far, show no evidence of malignancy.

Cervical smears (Papanicolaou) on all women were negative except for one patient in group I who subsequently was found to have adenocarcinoma of the uterus on curettage. Urine cytology was abnormal in four patients in group I and one patient in group II, showing dyskaryotic changes. Those patients demonstrating abnormal urine cytology have been followed and none has shown development of urinary tract neoplasms.

Alpha-fetoprotein testing was positive in one patient in group I, with no evidence of associated 
malignancy. All patients in group II were negative. CEA levels were negative in 15 patients tested in group I. In group II, 35 patients were tested and seven were positive. These seven people have been followed and show no evidence of malignancy. Family histories revealed a higher incidence of patients in group I who had first degree family members with diagnosed malignancies (15 vs six).

The occurrence of tumours in both groups was examined. In group I, seven patients had eight neoplasms, one of which, a thyroid adenoma, occurred before azathioprine was started. Hence, seven patients developed seven neoplasms after commencing azathioprine. Of these seven tumours three were malignant (table 4). For the seven patients the mean exposure to azathioprine was 1.6 years before diagnosis of their neoplasms. Four patients had a positive family history of malignancy.

In group II, five patients had six neoplasms in all, one of which was malignant. The tumours were: thyroid adenoma, lipoma, neurofibroma, two fibroids and a diffuse histiocytic lymphoma. None of these five patients had a positive family history of malignancy.

In terms of malignant and potentially malignant lesions, group I had three such tumours (two adenocarcinomas of the uterus, one carcinoma-insitu of the cervix) and group II had one diffuse histiocytic lymphoma. This difference did not reach statistical significance $(p=0.1728)$. The clinical and laboratory results of those patients with malignancies in group I did not differ significantly from group I as a whole.

Examination of marrow aspirates revealed a highly significant number of megaloblastic features in 28 of 36 patients studied in group $\mathrm{I}$, whereas only one out of 19 patients studied in group II showed such a change (table 5). Serum $B_{12}$ levels were similar in both groups, but serum folate levels, by both micro- biological and radiodilution assay, were significantly lower in group $\mathrm{I}$.

Analysis of marrow chromosomes performed in 25 of the 36 patients in group I and 11 of the 49 in group II showed striking differences in aberration index, percentage of cells with aberrations and aberration

TABLE 5 Laboratory features: erythropoietic factors

\begin{tabular}{llll}
\hline & $\begin{array}{l}\text { Azathioprine } \\
\text { treated (36) }\end{array}$ & $\begin{array}{l}\text { Control } \\
\text { (49) }\end{array}$ & Significance \\
\hline Marrow-megaloblastic & $28 / 36$ & $1 / 19$ & $\mathrm{p}<0.005$ \\
$\mathrm{~B}_{12}$ (ng/l) & 421 & 383 & $\mathrm{p}=0.63$ \\
Serum folate (ng/l) & 4.89 & 6.75 & $\mathrm{p}<0.025$ \\
${ }^{*}$ Serum folate (ng/l) & 4.76 & 7.05 & $\mathrm{p}=0.02$ \\
\hline
\end{tabular}

*Radiodilution.
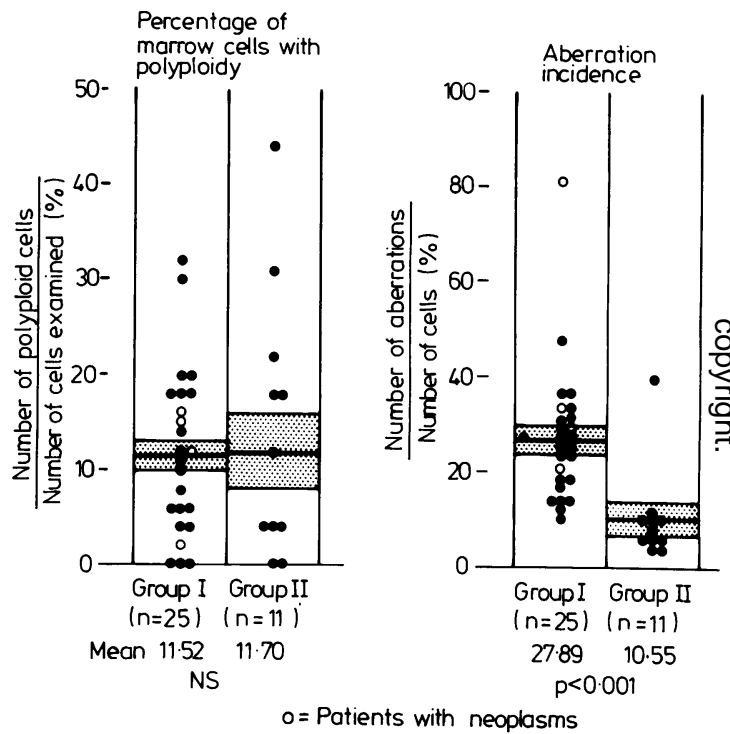

FIG 1 Marrow chromosomal analysis on 25 patients in group I and 11 in group II showing similar percentage ofcells with polyploidy but a higher aberration incidence in group I.

TABLE 4 Neoplasms in azathioprine treated patients

\begin{tabular}{|c|c|c|c|c|c|c|c|}
\hline Patient & Sex & $\begin{array}{l}\text { Age } \\
(y r)\end{array}$ & $\begin{array}{l}\text { Azathioprine } \\
\text { dose } \\
\text { (mg/kg/day) }\end{array}$ & $\begin{array}{l}\text { Duration of } \\
\text { azathioprine } \\
\text { (mth) }\end{array}$ & $\begin{array}{l}\text { Other } \\
\text { medications } \\
\text { at diagnosis } \\
\text { of cancer }\end{array}$ & Tumour & $\begin{array}{l}\text { Family } \\
\text { history }\end{array}$ \\
\hline JA & $\mathrm{F}$ & 44 & $1 \cdot 8$ & 34 & $\begin{array}{l}\text { Prednisone } \\
\text { Chloroquine }\end{array}$ & Ca-in-situ & + \\
\hline $\mathrm{PMcM}^{*}$ & $\mathbf{F}$ & 54 & 0.85 & 34 & Prednisone & $\begin{array}{c}\text { Adeno Ca } \\
\text { uterus }\end{array}$ & - \\
\hline DP & $\mathbf{F}$ & 47 & $1 \cdot 0$ & 12 & - & $\begin{array}{c}\text { Adeno } \mathrm{Ca} \\
\text { uterus }\end{array}$ & + \\
\hline JD & $\mathrm{F}$ & 50 & $1 \cdot 38$ & 20 & - & Haemangioma & + \\
\hline DD & $\mathrm{F}$ & 52 & $1 \cdot 16$ & 11 & - & $\begin{array}{l}\text { Benign liver } \\
\text { cyst }\end{array}$ & - \\
\hline MT & $F$ & 70 & $2 \cdot 02$ & 12 & - & Anal polyp & - \\
\hline $\mathrm{JH}$ & $\mathrm{F}$ & 47 & $0 \cdot 80$ & 12 & - & $\begin{array}{l}\text { Fibroadenoma } \\
\text { breast }\end{array}$ & + \\
\hline
\end{tabular}




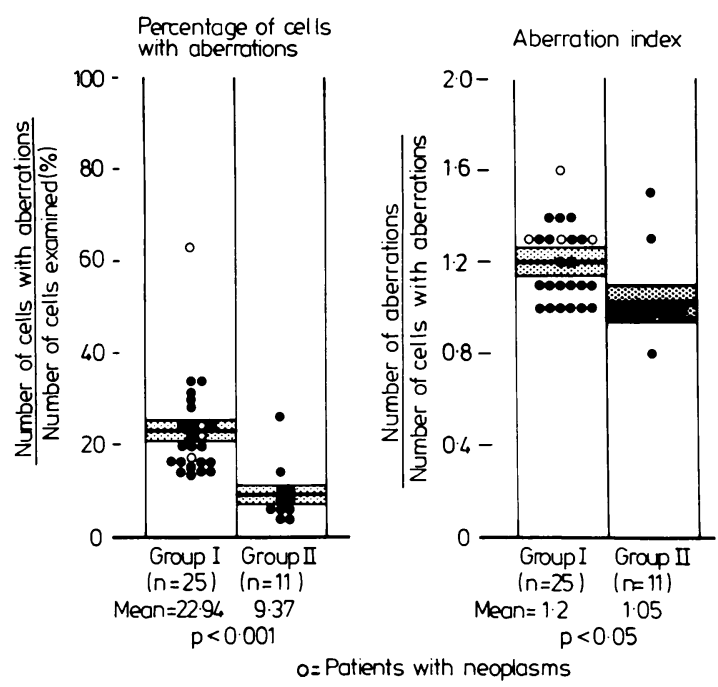

FIG 2 Marrow chromosomal analysis on 25 patients in group I and 11 in group II showing a greater percentage of cells with aberrations and a higher aberration index in group $I$.

incidence. The azathioprine treated group I showed more abnormal patterns (figs 1,2) in three of the four chromosomal variables measured. (Normal value in our laboratory for the aberration incidence is $10 \%$ or less.)

Four of the patients with neoplasms in group I showed a greater number of chromosomal abnormalities than the other 21 studied in the group. Two of these patients had benign and two malignant neoplasms. However, the two patients with malignancies differed from the remainder of group I in only one of the variables, the aberration index.

\section{Discussion}

The use of azathioprine in severely active RA, refractory to standard disease suppressants, has been shown to be effective in several studies, ${ }^{3 \times 124} 143224$ but potential neoplastic effects have remained a serious concern. There have been several case reports of malignancy in patients with RA on azathioprine alone, or in combination with other cytotoxics, ${ }^{23279125176177196}$ with a striking frequency of acute myelogenous leukaemia, occurring predominantly in males. ${ }^{79} 196$

Azathioprine was first shown to be associated with an increased incidence of malignancies when used in combination with prednisone in the management of organ transplants. ${ }^{175}{ }^{176}$ Although the reported incidence of this complication may be higher because of the possible overlapping incidence of malignancy during chronic renal failure, ${ }^{198}$ there is no doubt that organ transplant patients maintained on chronic immunosuppressive treatment have a significantly increased risk of developing de novo cancers within the first few years of transplantation. Almost all such patients received azathioprine and prednisone, as well as other immunosuppressive medications in several cases. This phenomenon has been variously explained by: the chronic presence of, and stimulation by, foreign transplanted antigen; immunosuppression sufficient to allow survival of spontaneous host tumours; and increased susceptibility to viral oncogenesis; or direct oncogenic effect by the immunosuppressive agent.

In more recent years, azathioprine has been used in many non-malignant conditions characterised as being of 'immunological' origin. Although Sharon et al's series of SLE patients ${ }^{198}$ and Worthington's observations (cited in Skinner and Schwartz ${ }^{202}$ ) of only one malignancy per 3000 such patients have been used to deny an increased incidence of neoplasms in patients treated with immunosuppressive agents, over 30 cases of malignancies have been reported in this group treated by azathioprine alone, or in combination with other cytotoxic agents. Many of these cases admittedly have a tenuous relationship to the drug in question. $8293261749011 \times 125127134141146157$ $\begin{array}{lllllllllllll}171 & 177 & 1 \times 1 & 1 \times 7 & 192 & 196 & 199 & 200 & 203 & 21 \times & 221 & 236 & 240\end{array}$

We have compared 36 patients with RA taking long-term azathioprine therapy (group I) with 49 age and sex matched patients not taking this medication (group II). The groups were similar in clinical and laboratory features except for a higher age and lower WBC counts in the azathioprine group, as previously reported. ${ }^{95} 225$

The outstanding finding was the demonstration of seven tumours in group I while they were taking azathioprine, three of which were malignant or premalignant. This was compared to five benign and one malignant tumour in group II. Of the 36 azathioprine treated patients in this study, only women developed neoplasms. The three malignant lesions were located in the genitourinary tract, a common site for neoplastic change in the elderly female, and the benign lesions were found in the skin, liver, breast, bowel and uterus. There was one malignant neoplasm identified in control group II, a diffuse histiocytic lymphoma. Previously reported prevalence rates of malignancy in RA have been either comparable to non-rheumatoid groups, ${ }^{166}$ or unexpectedly low $(0.6 \%) .{ }^{126}$ The finding of three malignancies among 36 azathioprine treated patients as compared with one among the 49 controls is not statistically significant $(p=0 \cdot 1728)$. Furthermore, statistics from the Ontario Cancer Research Foundation suggest that the expected incidence of malignan- 


\section{Suppl p 22 Annals of the Rheumatic Diseases}

cies over a three-year period in age matched females should be less than one case per 28 women $(0.75$ cases/28 women) (Ontario Cancer Treatment and Research Foundation). ${ }^{162}$ This is not significantly less than our finding of three cases per 28 women.

Significantly the diagnosis of neoplastic change in group I occurred early in the course of their treatment, within a mean of 1.6 years from the initiation of therapy, as compared to a mean duration of 3.5 years of azathioprine treatment in group I as a whole. It is of interest that an additional male patient in group I developed a malignant lymphoma while still taking azathioprine, one year after his assessment in this study, and two patients, already reported from this unit previously, developed acute leukaemia. No controls for these patients were available. On the other hand, the four patients with benign lesions continued to take azathioprine for a further mean period of 20 months and have suffered no malignant transformation nor further neoplasms. Of the three patients with malignancies, two have elected to continue azathioprine after treatment of their neoplasms and have suffered no recurrence nor further primary malignancies. This pattern would imply that a premalignant lesion may be present when azathioprine is initiated, and with immunosuppression the malignancy may manifest itself relatively early during the course of treatment. Carcinomas of the cervix are a common de novo cancer in renal transplant patients occurring in the first years after transplantation. ${ }^{176}$ Cervical dysplasia occurring as soon as 12 days after initiation of azathioprine therapy has been reported. ${ }^{85}$

Marrow chromosomal analysis has been shown to be a more effective means of determining cytogenic effects of agents than leucocyte chromosomal analysis. ${ }^{104}{ }^{144}$ We performed marrow chromosomal analysis and found an increased number of abnormalities in the azathioprine treated group despite the fact that the mean dose of azathioprine used was 1.08 $\mathrm{mg} / \mathrm{kg} / \mathrm{day}$, a low dose regimen. Nevertheless, chromosomal changes were significantly abnormal, but the number of abnormalities could be correlated neither to the dose nor to the duration of azathioprine treatment, a finding previously reported. ${ }^{105}$ Our results confirm previous reports of the clastogenic effect of azathioprine on chromosomes in nonmalignant disease. These changes have been reported to return to normal on discontinuation of the drug. ${ }^{95}{ }^{105}$ However, there was a marked degree of overlap between results in group I and group II. As well, this overlap was apparent when comparing results of those patients in group I with tumours and the remainder of group I. Although those with tumours had greater chromosomal abnormalities as a group, other subjects in group I, as well as in group II, had equally abnormal analyses when looked at individually (figs 1,2 ). Thus, the chromosomal analysis alone could not identify those individual patients with neoplasms. The development of tumours may reflect an increased susceptibility to the oncogenic potential of azathioprine in certain patients, a susceptibility possibly marked by a positive family history for malignancy (table 4).

Originally claimed to be a marker of bowel carcinoma, the CEA has now proven to be much less specific. None of the patients in the azathioprinetreated group had increased levels of CEA. Of interest, is the fact that seven of the control group had significant levels without evidence of any neoplasms. This finding of raised CEA levels in seropositive RA has been previously reported..$^{222} 223$

Alpha-fetoprotein is another oncofetal antigeno previously reported to be a potential marker for? hepatic carcinoma. ${ }^{4}$ We detected this antigen in only one patient in group I, who proved to have no evidence of an associated malignancy. All patients in group II were negative for alpha-fetoprotein.

The problem of therapy with azathioprine in RA and the increasing incidence of neoplastic disease resulting from such treatment is a serious consideration, but to date no registry has been organised to gather and analyse such cases in a similar manner to that performed for organ transplant malignancies. Without such an organisation, meaningful statistics and conclusions will inevitably be delayed. We conclude that the apparent increased risk, as demoristrated in this study, warrants further studies with larger populations and over a continuous longer period. 


\title{
Interim observations on benefit/risk of azathioprine versus $D$-penicillamine in the treatment of rheumatoid arthritis
}

\author{
JOHN R WARD* AND H JAMS WILLIAMS* \\ From the School of Medicine, University of Utah, Salt Lake City, Utah, USA
}

Azathioprine has been shown to be an effective drug for the treatment of rheumatoid arthritis. Levy et al ${ }^{124}$ have reported improvement in joint counts with azathioprine and Urowitz et al ${ }^{224}$ found azathioprine to be superior to placebo in reducing the articular index and joint counts. Under double-blind conditions, azathioprine, cyclophosphamide and gold produced comparable clinical improvement over 18 months of observation. ${ }^{38}$ In a single-blind external observer trial, azathioprine $2.5 \mathrm{mg} / \mathrm{kg} /$ day produced similar efficacy and toxicity when compared with D-penicillamine $1 \mathrm{~g} /$ day. ${ }^{1 \mathrm{t}}$ In a parallel uncontrolled open trial, azathioprine, gold and chloroquine were essentially equivalent in the control of disease activity in patients with early rheumatoid arthritis. ${ }^{56}$ Additional studies support the beneficial effects of azathioprine in rheumatoid arthritis. ${ }^{25} 1433225$

The selection of slow-acting antirheumatic drugs (SAARDs) for treatment still remains controversial. Our group considers gold to be the SAARD of first choice. However, for patients who fail to respond to gold, or develop toxicity, cytotoxic drugs or D-penicillamine represent the next therapeutic option. Thus, the current study was developed to compare the efficacy and toxicity of azathioprine and D-penicillamine in a prospective randomised controlled double-blind 24-week trial. The study will evaluate long-term efficacy and safety during a five-year non-blinded follow-up period, during which patients may continue to take one of the study drugs as long as it is clinically indicated.

\section{Materials and methods}

P A T I E N T S

A total of 200 patients will be entered. Patient selection criteria include the following.

(1) Definite or classical rheumatoid arthritis (ARA criteria) of greater than six months' duration.

*For the Cooperating Clinics, Cooperative Systematic Studies of the Rheumatic Diseases. Supported by NIAMDD Contract NO1-AM-6-2218 and a grant from Burroughs Wellcome Company.
(2) Onset after the age of 16 years.

(3) Active disease defined as having six or more swollen joints that are considered responsive to therapy and at least two of the following:

(a) Nine or more responsive joints tender on pressure;

(b) 45 minutes or more of morning stiffness;

(c) Westergren erythrocyte sedimentation rate of $28 \mathrm{~mm} / \mathrm{h}$ or greater.

(4) Failure to respond to at least $750 \mathrm{mg}$ parenteral gold or toxic reaction to gold.

(5) Inadequate control of arthritis with therapeutic doses of aspirin or other non-steroidal anti-inflammatory drugs (NSAIDs).

(6) Absence of childbearing potential in females.

(7) Absence of contraindication: hepatic disease, renal failure, proteinuria, thrombocytopenia or leucopenia

(8) No previous treatment with cytotoxic drugs.

(9) Constant optimal dose of aspirin, NSAID, and/or prednisone (10 $\mathrm{mg}$ or less/day).

(10) Informed consent.

T R E A T M E N T

A randomised schedule assigned 12 to 24 patients to each of 11 participating clinics, with equal numbers to receive azathioprine in doses of 1.25 to 1.5 $\mathrm{mg} / \mathrm{kg} /$ day or D-penicillamine in doses of 10 to 12 $\mathrm{mg} / \mathrm{kg} /$ day with a five-year follow-up. Each patient receives azathioprine tablets $(25 \mathrm{mg})$ and placebo capsules, or D-penicillamine capsules $(125 \mathrm{mg})$ and placebo tablets. The azathioprine is initiated to provide the full dose from start of trial, whereas D-penicillamine is increased by $250 \mathrm{mg}$ every four weeks until the full dose is reached and maintained for the 24 week duration of the double-blind phase.

MEASUREMENT OF DISEASE ACTIVITY For measurement of efficacy the following variables were measured at entry and every six weeks.

(1) Functional assessment.

(2) Duration of morning stiffness. 
(3) Onset of fatigue.

(4) Overall assessment of disease activity as estimated by both physician and patient.

(5) Joint counts for number of tender joints and tenderness score for joints $(0=$ none, $1=$ mild, $2=$ moderate, $3=$ severe).

(6) Joint counts for number of swollen joints and a swelling score.

Additional criteria for response include*:

(1) Remission.

(2) Significant clinical improvement.

(3) Overall meaningful clinical improvement.

(4) Subjective improvement.

(5) No response.

(6) Progressive disease.

Extra-articular manifestations, including subcutaneous nodules, skin ulcers, vasculitis, etc, will be evaluated.

\section{OTHER ASSESSMENTS}

Patients are seen every two weeks for evaluation of side-effects, monitoring of drug compliance and laboratory studies, which include complete blood count,

${ }^{*}$ Definitions will be provided in a report when the trial has been completed.

TABLE 1 Comparison of descriptive variable at entry for patients completing the trial by treatment group

\begin{tabular}{llcc}
\hline Variable & Measure & \multicolumn{2}{l}{ Treatment group } \\
\cline { 3 - 4 } & & $A(n=38)$ & $B(n=31)$ \\
\cline { 3 - 4 } & & 50 & 53 \\
Age (years) & Mean & 71 & 74 \\
Sex & Female (\%) & 84 & 90 \\
Race & Caucasian (\%) & 16 & 10 \\
& Black (\%) & 135 & 124 \\
Duration (months) & Mean & $2 \cdot 2$ & $2 \cdot 1$ \\
Functional class & Mean & $2 \cdot 2$ & $2 \cdot 1$ \\
Severity & Mean & & \\
\hline
\end{tabular}

TABLE 2 Median differences for measurement variables by treatment group between the conclusion and initiation of the trial* $^{*}$

\begin{tabular}{|c|c|c|}
\hline \multirow[t]{2}{*}{ Variable } & \multicolumn{2}{|c|}{ Treatment group } \\
\hline & $\boldsymbol{A}$ & B \\
\hline \multicolumn{3}{|l|}{$\begin{array}{l}\text { Functional class (scale } 1=\text { no disability, } \\
4=\text { incapacitated) }\end{array}$} \\
\hline Duration of morning stiffness (min) & $-1 \cdot 25$ & $-9 \cdot 0$ \\
\hline $\begin{array}{l}\text { Physician assessment (scale } 1=\text { no disease, } \\
4=\text { severe) }\end{array}$ & $0 \cdot 27$ & 0.73 \\
\hline Patient assessment (scale as above) & 0.68 & $0: 87$ \\
\hline Number of tender joints & $6 \cdot 50$ & $10 \cdot 33$ \\
\hline Joint tenderness score & $22 \cdot 00$ & $26 \cdot 00$ \\
\hline Number of swollen joints & $5 \cdot 00$ & $10 \cdot 00$ \\
\hline Joint swelling score & $5 \cdot 52$ & $7 \cdot 00$ \\
\hline \multicolumn{3}{|l|}{ Grip strength (mmHg) } \\
\hline Right hand & $8 \cdot 2$ & $15 \cdot 3$ \\
\hline Left hand & $5 \cdot 3$ & $13 \cdot 0$ \\
\hline
\end{tabular}

*Median change from entry to completion. A positive value indicates improvement. platelet count and urine analysis. A chemical survey and Westergren sedimentation rate is done every six weeks. Rheumatoid factor and antinuclear antibody tests are done at entry and completion.

\section{Results}

PATIENTS STUDIED

To date 148 of the 200 expected patients have been enrolled and 69 have completed the double-blind phase. Because the study is incomplete and still in progress, only blind comparisons of the two treatments are presented. The patients in each study group were comparable (table 1).

RESPONSE TO THERAPY

The results for selected measurements of disease activity are shown in table 2 . There was improvement in duration of morning stiffness, overall assessment of disease activity as judged by both physician and patient, number of tender joints and joint tenderness score and number of swollen joints and joint swelling score. The differences were similar but seemed to favour treatment B. No formal statistical testing has been done because of the ongoing nature of the trial

WIT H D R A W A L S

Of the 148 patients entered, 16 patients withdrew for the reasons shown in table 3 . More detailed reasons leading to withdrawal are shown in table 4 . Toxicity

TABLE 3 Reason for withdrawal of patients who have entered the trial to date

\begin{tabular}{lllll}
\hline Treatment & $\begin{array}{l}\text { No of patients } \\
\text { entered }\end{array}$ & \multicolumn{3}{l}{ No of patients withdrawn } \\
\cline { 3 - 5 } & & ADR & NR & UD \\
\hline A & 77 & 6 & 0 & 2 \\
B & 71 & 5 & 1 & 2 \\
\hline ADR =adverse drug reaction & & & \\
NR=non-responsiveness & & & \\
UD=unrelated to drug (moved, lack of co-operation, intercurrent illness, etc)
\end{tabular}

TABLE 4 Reason for withdrawal from study by treatment group

\begin{tabular}{lll}
\hline Reason for withdrawal & No of patients & \\
\cline { 2 - 3 } & Treatment $A$ & Treatment $B$ \\
\hline Thrombocytopenia & & 1 \\
Proteinuria & 2 & 1 \\
Leucopenia & 2 & 1 \\
Nausea & 1 & \\
Abnormal liver function tests & 1 & 1 \\
Itching and rash & & 1 \\
Oral ulcers & & 1 \\
Herpes zoster & & 1 \\
Inadequate response & 2 & 1 \\
Unrelated disease & & \\
Lack of co-operation & & \\
\hline
\end{tabular}


as determined by withdrawals was similar although from different causes for the two groups.

\section{Discussion}

Preliminary results of this study to compare the efficacy, safety and tolerance of selected doses of azathioprine and D-penicillamine have indicated improvement in rheumatoid arthritis with both treatments. However, the data to date would suggest better efficacy for treatment $B$. Until completion of the trial, blinding will be maintained. The null hypothesis states that one group is equal to the other group. Failure to reject the hypothesis when a real difference exists is termed a type II error, while rejection of the hypothesis when no real difference is present is a type I error. No formal statistical comparisons are presented because of the small sample size and thus the risk of committing a type II error. The trial was designed to detect a $30 \%$ difference between the treatment groups with $\alpha=0.05$ and $\beta=0 \cdot 1$, and allows for a dropout rate of $30 \%$. Until the 200 patients have completed the trial, only a striking difference in efficacy or toxicity will terminate the trial!

An important component of this study is to examine for a subset of unique responders. Thus, $a$ priori definitions of remission and varying degrees of clinical response and worsening were developed. Analysis of these subsets will also await completion of the formal trial.

The long-term follow-up of patients, who continue on their treatment for extended periods of time, is required to help the physician in the selection of treatment. Short-term efficacy is not always equatable to value of therapy, as tolerance and continued benefit are important.

It would have been far more useful to have been able to present not only the results of the double-blind comparison, but the long-term follow-up. While this is not possible, the ultimate results of this trial should help clinicians select which drug they judge would be helpful in the treatment of 'unresponsive' rheumatoid arthritis. 


\title{
An overview of benefit/risk of disease modifying treatment of rheumatoid arthritis as of today*
}

\author{
HAROLD E PAULUS \\ From the Department of Medicine, UCLA School of Medicine, Los Angeles, California 90024, USA
}

SUMMARY In chronic rheumatoid arthritis (RA), disease modifying drugs are used in an attempt to suppress the progressive damage to tissues and joints that is associated with active disease. Their success in achieving this goal is variable; responses vary from complete suppression of all signs and symptoms of RA to continued active disease, with progressive disability, despite prolonged therapy. Because disease activity almost always recurs after the therapy is stopped, early interruption of an effective therapy for any reason will make its benefit insignificant in a lifelong disease such as RA. Similarly, short-term sequential use of multiple disease modifying therapies is unlikely to be beneficial. The immediate problems with these therapies are substantial. In general, fewer than $50 \%$ of patients are able to continue a particular drug for more than one year. Since it takes three to 12 months or longer to achieve maximum effects, those patients who are unable to continue the drug receive little benefit from it. Inevitable delayed side-effects, such as those associated with chronic corticosteroid therapy, may make the benefit/risk ratio unacceptable. Potential late lethal adverse effects, such as malignancy, weight the benefit/risk ratio to varying extents for individual patients, depending on the relative probability that the adverse effect will occur during the remainder of the patient's anticipated life span, and are of greater importance in younger patients. In that minority of patients who achieve remission or near remission and are able to tolerate a disease modifying treatment for many years, it is of truly significant benefit. We are still searching for a therapy that will reliably achieve this goal for most patients with RA.

The purpose of disease modifying antirheumatic drug (DMARD) therapy of rheumatoid arthritis (RA) is to:

(1) reduce, suppress, or eliminate disease activity now; and

(2) thereby prevent the extended and perhaps permanent effects of disease activity. ${ }^{42}$

"Supported in part by USPHS Grant NO1-AM6-2218.
Currently available DMARDs include organic gold compounds, antimalarial drugs (chloroquine and hydroxychloroquine), D-penicillamine and the immunosuppressants (azathioprine, 6-mercaptopurine, cyclophosphamide, chlorambucil and methotrexate). Investigational disease modifying therapies include the $\mathrm{T}$ cell stimulants levamisole and thymopoietin, lymphocyte depletion or suppression by thoracic duct drainage, lymphopheresis, antithymocyte globulin, or total lymphoid irradiation and removal of plasma constituents by plasmapheresis.

These interventions are remarkably diverse in their chemical and pharmacological properties ando응 effects. Yet, they appear to be remarkably similar in their effects in patients with RA. Their administration does not result in any observable immediate amelioration of the signs or symptoms of RA, such as one expects to see with corticosteroids or nonsteroidal anti-inflammatory drugs. However, after weeks or months of treatment, a subtle and somewhat questionable decrease in the severity of symptoms may occur. With continued therapy, the average patient demonstrates overall moderate suppression of disease activity, but this average includes some patients who have no improvement at all, and others who eventually achieve complete, or nearly complete, remission of disease manifestations, often accompanied by normalisation of laboratory abnormalities that are associated with disease activity, and stabilisation of $x$-ray evidence of joint damage. However, these beneficial effects are not permanent. When the disease modifying treatment is stopped benefit usually persists for weeks or months, but then disease manifestations gradually recur in the same delayed and subtle fashion as they had disappeared when the therapy was started.

How well do we succeed in our goal of preventing the progressive damage to tissues and joints that is associated with disease activity using the DMARDs that are available today? What price do our patients pay for these benefits?

Chronic RA is usually a life-long disease. If we expect to restore the patient to normal, productive 
life with a DMARD induced remission, that remission must be sustained for many years. Since it generally takes three to 12 months or longer to achieve maximum benefit with DMARD therapy, patients who are unable to take the drug for more than one year receive little benefit from it.

\section{Benefits}

In evaluating the long term benefits of DMARDs on the life quality of patients with RA, the classic published placebo-controlled double-blind studies that have established the use of these drugs are of little value because, in order to avoid unnecessarily prolonged administration of placebo, they are generally of the shortest possible duration, usually no more than four to 12 months. $^{346371140155224}$ However, there are some published reports on the long-term usefulness of some of the DMARDs.

\section{GOLD COMPOUNDS}

Srinivasan $e t a^{205}$ reported our findings in a cohort study of 111 patients who were followed for an average of three years after starting gold therapy. Fiftyeight $(52 \%)$ patients stopped gold within the first 18 months. Of those who continued gold for more than 18 months, $83 \%$ achieved remission, although five of these subsequently flared while continuing gold therapy. Thus, of the 111 patients starting gold, about $35 \%$ had major sustained improvement an average of three years later.

Rothermich and associates ${ }^{189}$ started 97 patients in a prospective study of gold therapy. After an average of $1 \frac{1}{2}$ years, $41 \%$ were having sufficient clinical benefit to continue the drug. However, four to six years after the study was started, only 14 patients were continuing gold therapy, and two more had discontinued because of remission. Most stopped gold because of loss of benefit, or toxicity, or both simultaneously.

In the same report, Rothermich et al ${ }^{189}$ examined 171 patients who started D-penicillamine after failing gold therapy and $53 \%$ had stopped gold within the first year, more for toxicity than for lack of benefit. At the end of three years, only $20 \%$ continued gold, somewhat fewer than Srinivasan's finding of $35 \% .{ }^{205}$ At the end of five years, only $9 \%$ were still taking gold. Of course, these figures are biased by the requirement that only patients who had stopped gold were counted; the analysis is completely retrospective and the population may not be representative. Nevertheless, the majority of these patients stopped gold for loss of benefit.

\section{D-PENICIL LAMINE}

Several similar studies of D-penicillamine therapy have been reported recently. Kean et al ${ }^{112}$ found that $47 \%$ of 101 patients continued D-penicillamine after one year and $38 \%$ after two years. The major reason for stopping therapy in the first year was toxicity. After two years, two patients were in remission, five in partial remission, and 31 had $75 \%$ improvement.

Webley and Coomes ${ }^{233}$ reported that $46 \%$ of 114 patients continued penicillamine after an average of $10 \cdot 1$ months' treatment. Of the 62 withdrawals, 40 were for toxicity and 12 for lack of response. There were no differences in toxicity or improvement rates in patients receiving more or less than $600 \mathrm{mg}$ daily, or in patients who had or had not had previous gold therapy.

\section{COMPARATIVE STUDIES}

Husain and Runge ${ }^{96}$ evaluated the risks of termination of treatment with gold, penicillamine, hydroxychloroquine and levamisole using a life table analysis. The risks for termination of gold and penicillamine were similar to those reported above, while that for hydroxychloroquine ( $25 \%$ in 12 months) was lower, and that for levamisole (57\% in 12 months) higher than for the other two drugs. However, in another paper, the same group reported that the median termination time was 60 months for gold and 13 months for antimalarial compounds. Lack of efficacy was responsible for treatment termination in $41 \%$ of the antimalarial group in this study in which treatment was frequently terminated for lack of response after three to six months of treatment. ${ }^{185}$

Dwosh et $a l^{56}$ randomly assigned patients with class II RA to azathioprine, gold or chloroquine. After six months of treatment, all three agents were comparable in terms of toxicity and clinical improvement. However, the chloroquine group did not maintain continued improvement beyond six months, while in the gold and azathioprine groups benefit continued for up to 44 months.

Hoh et $a l^{93}$ reported the incidence of remissions of RA in patients treated with gold, penicillamine, or chloroquine for at least one year. Prolonged remissions occurred in only $10 \%$ of the 230 drug courses, while $59 \%$ had modest partial responses and the remainder had no response.

Currey et $a l^{38}$ compared azathioprine, cyclophosphamide and gold in a well-designed 18-month double-blind randomised trial of 121 patients. They concluded that the two immunosuppressives produced clinical improvement comparable to that with gold. Cyclophosphamide was perhaps marginally more effective. Drug management was easiest with azathioprine. However, only $25 \%$ of patients on azathioprine, $36 \%$ on cyclophosphamide and $24 \%$ on gold completed 18 months of treatment. Of course, withdrawals for toxicity were increased by the 
double-blind nature of the trial and the inclusion of gold, which necessitated withdrawing seven azathioprine and 11 cyclophosphamide patients for marrow depression.

On the other hand, Cade et $^{2}{ }^{25}$ reported that 14 of 16 patients treated with azathioprine for two to five years achieved $100 \%$ work ability, although it took up to 32 months of treatment to achieve that maximum response. Furthermore, serial $x$-rays showed no progression of destruction after completion of the second year of therapy, even when followed for as long as eight years.

\section{Risks}

Only major risks will be evaluated here. It is obvious that patients who are forced to stop treatment because of temporary reversible rash, proteinuria, marrow suppression, drug induced hepatitis, etc, do not benefit from the drug, but these adverse reactions rarely shorten their life span or produce permanent damage.

\section{E A T H}

Davis $^{40}$ has recently reviewed the undesirable effects of gold therapy. With current dosages, marrow aplasia is the most serious adverse effect. Over a seven-year period in Great Britain, 16 deaths related to gold therapy were found, an estimated incidence of 1.6 deaths per 10000 prescriptions. This figure suggests to Davis that gold is ten times more toxic than any other therapy used in Great Britain. Davis reviewed four studies, published in 1962, 1968, 1976 , and 1977 , reporting a total of 50 patients with gold induced marrow aplasia, which was fatal in $66 \%$.

Similarly, Kay ${ }^{111}$ collected information on ten patients in Great Britain with pancytopenia associated with penicillamine therapy, six of whom died. The dose range was 250 to $1000 \mathrm{mg} /$ day with an average of $615 \mathrm{mg}$. The duration of therapy was from three to 60 months with a mean of 16 months. She reports that the Committee of Safety of Medicines had had reported to them 18 deaths from D-penicillamine from 1964 to 1977 (three of whom were included in the six deaths reported by Kay). Fourteen of the 18 deaths were due to blood dyscrasias.

Similarly, deaths have occurred in patients who have developed agranulocytosis while taking levamisole. ${ }^{147}$

Lewis et al ${ }^{125}$ reported on the causes of death in 311 patients with RA who were observed over an 11-year period. A total of 46 patients died, 13 of them from malignancies. The incidence of deaths from neoplasia was not greater in 214 patients treated with azathioprine and/or chlorambucil or cyclophosphamide
$(2.8 \%)$ than in 97 patients who did not receive a cytotoxic drug $(7 \%)$.

Isomaki et $a l^{102}{ }^{101}$ reported two large studies of causes of death in patients with RA in Finland. In the first, an age and sex matched comparison of 1000 patients with RA and 1000 control subjects, malignant neoplasms were less frequent in RA (9\% of deaths; $1.1 \%$ of the population) than in the controls ( $30 \%$ of deaths; $2.1 \%$ of the population). In the second study of more than 45000 patients with RA, the risk of death from lymphoma, leukaemia or myeloma was twice as great in RA (130 deaths) as that expected in the general population (59 deaths). No data are given regarding the drugs used by these patients.

O THE R S ERIOUS TOX I C ITIE S

Maculopathy occurred in $10 \%$ of 222 patients treated with $800 \mathrm{mg} /$ day hydroxychloroquine or 500 $\mathrm{mg} /$ day chloroquine by Dubois for systemic lupus erythematosus, but after 10 years of follow-up, visual acuity was adequate in all but two of the patients. ${ }^{21}$ However, with $400 \mathrm{mg}$ daily for more than one year in 99 patients, no loss of vision occurred. ${ }^{190}$

Sterility occurs with cyclophosphamide and chlorambucil, haemorrhagic cystitis with cyclophos- phamide and hepatic fibrosis with methotrexate. All of the immunosuppressive drugs are thought to increase susceptibility to infection, particularly when combined with large doses of corticosteroids.

Induction of autoimmune diseases has been reported with penicillamine therapy for RA, and also for Wilson's disease. In addition, the renal lesions associated with gold induced proteinuria have the characteristics of an immune complex mediated nephropathy, and the agranulocytosis of levamisole appears to be immune mediated. We have also seen new autoimmune manifestations develop during the apparently successful treatment of RA with thoracic duct lymphocyte depletion. These observations suggest that, while correcting some disease related abnormalities of the immune system, we may perturb the system in such a way that other manifestations of autoimmune disease emerge in some patients.

\section{Conclusions}

For those patients who achieve remission with a DMARD and are able to tolerate it for many years, the benefit clearly is greater than the risk. Unfortunately, because most patients do not achieve optimal benefits, or must stop treatment for annoying sideeffects, our goal of favourably altering the course of their RA is not achieved. Except for the antimalarials, there appears to be a real, although small, risk of drug associated death with all of the available 
DMARDs. However, the available data are not suffi-. cient to produce a satisfactory rank order of this risk with the various drugs.

We have no reason to be satisfied with our present accomplishments with DMARD therapy. However, it seems clear that DMARDs affect either the cells that participate in immune responses or their products. With increasing knowledge about this system and its methods of communication, and increasing attention to the antigens associated with autoimmune diseases, perhaps future DMARDs will be more effective. 


\title{
EULAR register of patients on immunosuppressive drugs
}

\author{
A $\mathrm{KAY}^{*}$ \\ From the ARC Epidemiology Research Unit (Clinical Section), Department of Rheumatology, Guy's \\ Hospital, London SE1
}

A collaborative United Kingdom/Australasian study of cancer in 5172 patients treated with immunosuppressive drugs ${ }^{115}$ showed an excess of non-Hodgkin's lymphoma, squamous cell skin cancer, and possibly mesenchymal tumours, in a series of patients with and without transplants. The 1349 non-transplant patients in the series, which included 492 with rheumatoid arthritis or ankylosing spondylitis, showed less marked excesses of the same tumours. In other studies, leukaemias have been reported in non-transplant patients exposed to cyclophosphamide. ${ }^{107}$

The purpose of the European League Against Rheumatism (EULAR) Study is to register and follow-up a substantial number of patients with connective tissue diseases who are, or have been, treated with immunosuppressive drugs, in order to determine the subsequent malignancy rate.

Seventy-seven rheumatologists $\dagger$ in 13 member countries registered 716 patients treated with immunosuppressive drugs between January 1979 and December 1980: registration of patients continues. The 478 patients who started immunosuppressive treatment on or after 1 January 1979 form a complete prospective sample. The 238 patients already taking immunosuppressives on 1 January 1979 are an incomplete sample but include patients with a longer drug exposure who may provide useful 'early-warning' information.

Of the 716 patients, 220 are male and 494 are female (two sex not stated). The mean age of the patients at the time of registration was 55 years (range six to 85). The conditions for which immunosuppressive drugs were given are shown in table 1. Azathioprine was the most commonly used drug (table 2) except in the treatment of polyarteritis nodosa, for which cyclophosphamide was more frequently used (table 3 ).

Seven malignancies have been reported so far (table 4) but it is not possible yet to estimate the malignancy rate. Follow-up of the patients continues

*On behalf of EULAR Standing Committee on International Clinical Studies and Therapeutic Trials. and the first full analysis of the findings will be undertaken in 1983.

TABLE 1 Conditions for which immunosuppressive drugs were given

\begin{tabular}{lcr}
\hline & $\begin{array}{l}\text { No of patients } \\
\text { on register }\end{array}$ & $\%$ \\
\hline Rheumatoid arthritis (RA) & 581 & 81 \\
Systemic lupus erythematosus (SLE) & 35 & 5 \\
Psoriatic arthropathy (PsA) & 20 & 3 \\
Polyarthritis undefined & 11 & 2 \\
Polyarteritis nodosa (PAN) & 7 & 1 \\
Polymyositis (Pm) & 7 & 1 \\
Polymyalgia & 5 & 1 \\
Other & 40 & 6 \\
\hline
\end{tabular}

TABLE 2 Immunosuppressive drugs and frequency of usage: number of patients and (percentage)

\begin{tabular}{lccc}
\hline Drugs & $\begin{array}{l}\text { Prospective } \\
\text { sample }\end{array}$ & $\begin{array}{l}\text { Retrospective } \\
\text { sample }\end{array}$ & All patients \\
\hline Azathioprine & $288(60 \%) \ddagger$ & $159(67 \%)$ & $447(62 \%)$ \\
Cyclophosphamide & $105(22 \%)$ & $50(21 \%)$ & $155(22 \%)$ \\
Chlorambucil & $39(8 \%)$ & $27(11 \%)$ & $66(9 \%)$ \\
Proresid & $36(8 \%)$ & 0 & $36(5 \%)$ \\
Methotrexate & $9(2 \%)$ & $2(1 \%)$ & $11(2 \%)$ \\
Unknown & 1 & & 1 \\
\hline
\end{tabular}

$\ddagger$ Three patients were treated with azathioprine combined with another immunosuppressive drug ( 1 cyclophosphamide, 1 chlorambucil, 1 proresid).

TABLE 3 Immunosuppressive drugs used in relation to main conditions treated (see table 1 for abbreviations)

\begin{tabular}{lrrrll}
\hline & $R A$ & $S L E$ & $P S A$ & $P A N$ & $P m$ \\
\hline Azathioprine & 368 & 28 & 12 & 1 & 3 \\
Cyclophosphamide & 132 & 5 & 1 & 6 & 2 \\
Chlorambucil & 61 & 0 & 0 & 0 & 0 \\
Proresid & 33 & 0 & 1 & 0 & 0 \\
Methotrexate & 0 & 1 & 5 & 0 & 1 \\
\hline
\end{tabular}

TABLE 4 Type of malignancy reported in relation to diagnosis, sex, and immunosuppressive drugs

\begin{tabular}{llll}
\hline Diagnosis & Sex & Drug & Tumour \\
\hline RA & F & Cyclophosphamide & Acute myeloid leukaemia \\
RA & M & Azathioprine & Reticulum cell sarcoma \\
RA & M & Azathioprine & Ca bronchus (oat cell) \\
RA & M & Azathioprine & Ca bronchus \\
RA & F & Azathioprine & Ca cervix \\
RA & F & Azathioprine & Ca head of pancreas \\
\hline
\end{tabular}


$\doteqdot$ CONTRIBUTORS (1979 AND 1980)

Dr Bastin (Belgium)

Dr Brath (Norway)

Dr Bruckner (UK)

Dr Capell (UK)

Dr Cochrane (UK)

Dr Crouzet (France)

Dr Essigman (UK)

Professor Franchimont (Belgium)

Dr Gibson (UK)

Dr Golding (UK)

Professor Gullberg (Sweden)

Dr Haslock (UK)

Dr Hilton (UK)

Dr Huber-Bruning (Holland)

Professor Jayson (UK)

Professor Keitel (DDR)

Dr Mattingley (UK)

Dr Myles (UK)

Dr Nilson (UK)

Professor Panayi (UK)

Dr Rosenthal (Switzerland)

Dr E E Smith (UK)

Dr Stojanovic (Yugoslavia)

Dr Thompson (UK)

Dr Wiik (Denmark)

Dr Wysocka (Poland)
Dr Beardwell (UK)

Dr Brewis (UK)

Dr Budimir (Yugoslavia)

Dr Cardoe (UK)

Dr Colston (UK)

Dr Darlington (UK)

Professor Fehr (Switzerland)

Professor Frizziero (Italy)

Dr Glick (UK)

Dr Grahame (UK)

Dr Haavik (Norway)

Dr Helin (Denmark)

Dr Holden (UK)

Dr Huge (DDR)

Dr Jenni (Switzerland)

Dr Klafkl (DDR)

Dr Mennet (Switzerland)

Dr Newton (UK)

Dr Nissila (Finland)

Dr Popert (UK)

Dr Schwarz (Switzerland)

Dr P J Smith (UK)

Dr Sydnes (Norway)

Dr Webb (UK)

Dr Wojtulewski (UK)

Dr D Yates (UK)
Dr Boyle (UK)

Dr M Brown (UK)

Dr Camp (UK)

Dr Carty (UK)

Dr Corbett (UK)

Professor Deshayes (France)

Dr Feldman (UK)

Professor Gerber (Switzerland)

Dr Glyn (UK)

Dr Griffiths (UK)

Dr Hansen (Denmark)

Dr Hill (UK)

Dr Holt (UK)

Dr Jacoby (UK)

Professor Kahn (France)

Dr Kojander (Finland)

Dr Munthe (Norway)

Dr Nicholls (UK)

Professor Orloff (Belgium)

Dr Remans (Belgium)

Dr Sharp (UK)

Dr Steinberg (UK)

Dr Symons (UK)

Dr Webley (UK)

Dr Woodland (UK) 


\title{
The incidence of malignant disease in patients receiving cytotoxic therapy for rheumatoid arthritis
}

\author{
ALLAN B KIRSNER, STEPHEN J FARBER, ROBERT P SHEON \\ AND ROBERT I FINKEL \\ From the Division of Rheumatology, The Toledo Clinic, and the Medical College of Ohio, Toledo, Ohio, \\ USA
}

SUMmaRY One hundred and twenty-six patients with definite or classical rheumatoid arthritis admitted to hospital between 1965 and 1974 for cytotoxic therapy were studied for the presence and type of malignant disease. Each of the cytotoxic treated patients was age and sex matched to a rheumatoid arthritis patient admitted to hospital during the same years but who did not receive cytotoxic therapy. There was no increase in malignancy in the cytotoxic treated group over the control group.

The introduction of cytotoxic drugs in the treatment of rheumatoid arthritis (RA) has raised concern about haematological and other malignancy in these patients. Since our initial report ${ }^{32}$ of acute leukaemia occurring in a patient with rheumatoid arthritis who was treated with cytotoxic therapy (CT), several papers have been published documenting the occurrence of malignancy in cytotoxic treated patients. ${ }^{20} 2730127177178196236$

In this paper, we present a retrospective age, sex and duration of disease matched study of the incidence of malignancy in patients with rheumatoid arthritis receiving cytotoxic therapy.

\section{Materials and methods}

One hundred and twenty-six patients with definite or classical RA, admitted to hospital between 1965 and 1974 for cytotoxic therapy, were matched by computer for age, sex and duration of disease to a group of patients admitted to hospital with definite or classical RA who were not treated with cytotoxic therapy (table 1). Groups differed in that the cytotoxic treated group had more active aggressive disease. Rheumatoid nodules were present in 57 of the cytotoxic treated patients versus 37 control patients $(\mathrm{p}<0.05)$. The mean rheumatoid factor titre (latex tube dilution) was 1:282 in the non-cytotoxic treated patients and 1:970 in the cytotoxic treated patients $(\mathrm{p}<0.001)$. Acute phase indices (erythrocyte sedimentation rate and protein bound hexoses) were greater in the cytotoxic treated group (table 2). Patient follow-up from entry into the study was $4 \cdot 7$

TABLE 1 Study population

\begin{tabular}{|c|c|c|c|c|c|c|c|c|}
\hline & \multicolumn{2}{|l|}{ No } & \multicolumn{2}{|c|}{$\begin{array}{l}\text { Duration of } \\
\text { disease at } \\
\text { entry into } \\
\text { study }(y r)^{*}\end{array}$} & \multicolumn{2}{|c|}{$\begin{array}{l}\text { Age at } \\
\text { onset of } \\
\text { disease }(y r)^{*}\end{array}$} & \multicolumn{2}{|c|}{$\begin{array}{l}\text { Age at } \\
\text { entry } \\
\text { into } \\
\text { study }(y r)^{*}\end{array}$} \\
\hline & $M$ & $F$ & $M$ & $F$ & $M$ & $F$ & $M$ & $F$ \\
\hline $\begin{array}{c}\text { Control RA } \\
\text { patients }\end{array}$ & 41 & 85 & $6 \cdot 3$ & 9.0 & 50.5 & $47 \cdot 7$ & 56.9 & 56.7 \\
\hline $\begin{array}{l}\text { Cytoxic treated } \\
\text { RA patients }\end{array}$ & 41 & 85 & 56 & 111 & 501 & 45.4 & $55 \cdot 7$ & 56.4 \\
\hline
\end{tabular}

*Average.

TABLE 2 ESR and acute phase reactants

\begin{tabular}{lll}
\hline ESR & Wintrobe & Westergren \\
Control & 36.6 & 46.0 \\
CT treated & 42.4 & 62.8 \\
& $0.05<\mathrm{p}<0 \cdot 10$ & $\mathrm{p}<0.001$ \\
\hline Protein bound & & \\
$\quad$ hexoses & $($ Normal $=80-160)$ & \\
Control & 161 & \\
CT treated & 181.9 & \\
& $\mathrm{p}<0.001$ & \\
\hline
\end{tabular}

TABLE 3 Results

\begin{tabular}{cllll}
\hline & $\begin{array}{l}\text { Malignant disease } \\
\text { before study }\end{array}$ & $\begin{array}{l}\text { Malignant disease } \\
\text { following entry } \\
\text { into study }\end{array}$ & \\
\hline Control & Basal cell carcinoma & 1 & Basal cell carcinoma & 3 \\
RA patients & Leukaemia & 1 & Breast carcinoma & 3 \\
& Breast carcinoma & 1 & Lung carcinoma & 1 \\
& Cervical carcinoma & 1 & Carcinomatosis & 1 \\
& Uterine carcinoma & 1 & Lymphoma & 1 \\
Cytoxic treated & Basal cell carcinoma & 2 & Basal cell carcinoma & 4 \\
RA patients & Breast carcinoma & 3 & Lung carcinoma & 3 \\
& Colon carcinoma & 1 & Carcinomatosis & 1 \\
& Rectal carcinoma & 1 & Stomach carcinoma & 1 \\
\hline
\end{tabular}


years for the control group and 4.9 years for the cytotoxic group. Among the cytotoxic treated patients, 105 received cyclophosphamide and/or nitrogen mustard; 17 received one or both of these in combination with chlorambucil, thiotepa, mathotrexate or azathioprine; and four received either chlorambucil, thiotepa or azathioprine alone.

\section{Results}

Five control patients and seven cytotoxic treated patients had malignancies before entry into the study (table 3). Nine new malignancies developed in the control patients following entry into the study compared with nine new malignancies in the cytotoxic treated patients. There was a preponderance of skin and lung carcinomas in both the control and cytotoxic treated groups. Acute leukaemia did not occur in the cytotoxic treated patients, and our initial case report of acute granulocytic leukaemia ${ }^{32}$ remains the only haematological malignancy that occurred in our patients with rheumatoid arthritis on cytotoxic treatment. No patient developed bladder carcinoma.

\section{Discussion}

Cytotoxic drugs are commonly used in the treatment of connective tissue disease. 20273032127177178196236 Following our initial report, Seidenfeld et al ${ }^{196}$ described two patients treated with cytotoxic drugs for rheumatoid arthritis, who subsequently developed acute leukaemia. Chromosomal abnormalities were noted in both patients, and a sideroblastic anaemia developed in both patients before the development of leukaemia. The author suggested that the onset of sideroblastic anaemia, chromosomal damage and a markedly decreased ability to form colonies in marrow culture, were indications for the discontinuance of cytotoxic therapy.
Pinals ${ }^{177}$ reported 25 patients receiving azathioprine in the treatment of chronic polyarthritis. Two patients with rheumatoid disease were found to have carcinoma of the breast and lung; both were treated with azathioprine. Pinals proposed that the early appearance of malignancy suggested that the role of azathioprine may have been to permit acceleration of tumour growth rather than to induce malignant transformation.

Boyle $e t{ }^{20}{ }^{20}$ reviewed the incidence of malignancy in 27 patients ten years after cyclophosphamide therapy for rheumatoid arthritis. Three patients had died, one of adenocarcinoma of the breast. Three additional solid tumours were identified. One patient had ductal carcinoma of the breast, another had adenocarcinoma of the stomach and a third had carcinoma of the uterus. There were three squamous cell carcinomas. Compared to a population of age and sex matched controls, there was no increased incidence of malignancy in their patients. However, they noted that the two carcinomas of the breast approached a statistically significant increased incidence.

Plotz et al ${ }^{178}$ studied 11 patients treated with cyclophosphamide for rheumatoid arthritis. Anaplastic transitional cell carcinoma of the bladder was found in one patient with rheumatoid arthritis five years after stopping a therapeutically successful and uncomplicated course of $50 \mathrm{~g}$ cyclophosphamide. They suggested that the development of cystitis and bladder carcinoma was a strong argument for limited use of cyclophosphamide in non-malignant inflammatory rheumatoid conditions.

With the exception of our initial case report, none of our cytotoxic treated patients developed haematological malignancy or bladder carcinoma. Nine patients in the control group and nine patients in the CT group developed malignancy. Thus, we have not been able to document an increased incidence of malignancy in cytotoxic treated patients with rheumatoid arthritis. 


\title{
Excess risk of lymphomas, leukemia and myeloma in patients with rheumatoid arthritis*
}

\author{
H A ISOMÄKI, T HAKULINEN AND U JOUTSENLAHTI \\ From the Rheumatism Foundation Hospital, 18120 Heinola 12; the Finnish Cancer Registry, 00170 \\ Helsinki 17; and the Social Insurance Institution of Finland, 00250 Helsinki 25, Finland
}

SUMMARY The incidence of malignant neoplasms among 11483 male and 34618 female individuals with rheumatoid arthritis was studied using two separate nationwide data registers covering the whole Finnish population: the Social Insurance Institution's Population Data Register, which includes information on medication for certain chronic diseases, and the Finnish Cancer Registry, with data on all cancer patients diagnosed in Finland. The follow-up comprised a total of 213911 person years. The total incidence of all malignant neoplasms was higher in males and on the level expected in females. The expected number of cases of leukemia, lymphomas, Hodgkin's disease and myeloma in both sexes was 59.6 as compared with the 130 cases observed. This difference is statistically highly significant $(\mathrm{p}<0.001)$. The incidence of cancer of the respiratory organs was higher in males, and the incidence of cancer of the rectum and stomach lower than expected in rheumatoid females.

Conflicting evidence has been presented on the risk of malignant neoplasms in patients with rheumatoid arthritis (RA). Moesman ${ }^{151}$ found cancer to be twice as common in rheumatoid patients as in the general population. On the other hand, the proportion of malignant neoplasms as a cause of death in patients with rheumatoid arthritis was lower than that in controls matched for sex and age. ${ }^{102}$ Cancer was underrepresented in two autopsied series of rheumatoid arthritis. ${ }^{13}{ }^{156}$ Lewis et al ${ }^{126}$ found that cancer was less common in hospitalized RA patients than in patients with hypertension.

Special attention has been paid to the simultaneous occurrence of lymphoproliferative neoplasms and inflammatory rheumatic diseases such as RA. ${ }^{26} 29149234$ Oleinick $^{161}$ studied the literature in English published up to December 1965 . He collected a series of 951 RA patients with 9346 person

\footnotetext{
*Reprinted from J Chron Dis 1978; 31: 691-6.
}

years at risk from published case reports. No support was found for the hypothesis that there is an increased risk of leukemia or lymphoma in RA patients. Miller ${ }^{149}$ found the same prevalence of RA in patients with solid tumors $(0.42 \%)$ or lymphoproliferative neoplasms $(0.38 \%)$ as in the general American population $(0.38 \%)$.

It is possible that the immunological abnormality in RA patients increases the risk of contracting leukemia, lymphoma, or myeloma. The case reports cannot, however, resolve this question. The potential significance of this hypothesis in an understanding of the pathogenesis of these malignancies warrants further investigation. ${ }^{161}$

In this study the incidence of cancer in a large series of patients with rheumatoid arthritis was evaluated.

\section{Patients and methods}

The incidence of malignant neoplasms in rheumatoid arthritis patients was studied by making use of two computerized nationwide data registers, the Social Insurance Institution's Population Data Register and the Finnish Cancer Registry.

The Social Insurance Institution's Population Data Register has included information since 1965 on patients with chronic diseases entitling them to reimbursable medicines. One such disease is rheumatoid arthritis. The category 'rheumatoid arthritis' in this register also includes systemic connective tissue diseases (before 1970) and ankylosing spondylitis (from 1970 onwards). In 1974, subjects with ankylosing spondylitis accounted for $2.2 \%$ of all patients and those with systemic connective tissue diseases for $1.7 \%$ of all patients in the register. ${ }^{170}$

The Finnish Cancer Registry ${ }^{213}$ was started in 1952, and according to established health service practice, every cancer case is reported to this registry. Reporting has been obligatory since 1961 . The registry receives information from multiple sources: from hospitals (at various stages of the disease), from practitioners, and from pathological laboratories. Moreover, the registry makes an annual screening of 
all death certificates issued in the country, and thus is informed of the death of cancer patients from both cancerous and non-cancerous causes. On average, five notifications are received per case.

The patients entitled to fully reimbursable medication because of RA during the period 1967-1973 were retrieved from the Social Insurance Institution's Population Data Register. A total of 11483 male and 34618 female RA patients were identified.

This RA patient series was matched against the Finnish Cancer Registry. The series was followed up for new cancer cases on or after 1 January 1967, from

TABLE 1 All malignant neoplasms in males by age

\begin{tabular}{lrrrr}
\hline Age & $\begin{array}{l}\text { Number of } \\
\text { rheumatoid } \\
\text { patients }\end{array}$ & $\begin{array}{l}\text { Person years } \\
\text { at risk }\end{array}$ & Observed & Expected \\
\hline $0-4$ & 34 & $38 \cdot 1$ & 0 & $0 \cdot 0$ \\
$5-9$ & 94 & $234 \cdot 3$ & 1 & $0 \cdot 0$ \\
$10-14$ & 107 & $384 \cdot 5$ & 0 & $0 \cdot 0$ \\
$15-19$ & 151 & $575 \cdot 8$ & 0 & $0 \cdot 1$ \\
$20-24$ & 340 & $1061 \cdot 4$ & 1 & $0 \cdot 2$ \\
$25-29$ & 457 & $1649 \cdot 8$ & 0 & $0 \cdot 4$ \\
$30-34$ & 611 & $2201 \cdot 4$ & 1 & $0 \cdot 8$ \\
$35-39$ & 772 & $2948 \cdot 6$ & 2 & $1 \cdot 8$ \\
$40-44$ & 1063 & $3920 \cdot 3$ & 7 & $4 \cdot 3$ \\
$45-49$ & 1181 & $4997 \cdot 8$ & 11 & $10 \cdot 1$ \\
$50-54$ & 1397 & $5659 \cdot 7$ & 24 & $21 \cdot 7$ \\
$55-59$ & 1760 & $7301 \cdot 4$ & 60 & $48 \cdot 7$ \\
$60-64$ & 1691 & $7855 \cdot 0$ & 95 & $85 \cdot 2$ \\
$65-69$ & 998 & $5674 \cdot 3$ & 99 & $83 \cdot 4$ \\
$70-74$ & 515 & $2883 \cdot 3$ & 50 & $56 \cdot 2$ \\
$75-79$ & 222 & $1262 \cdot 2$ & 42 & $29 \cdot 0$ \\
$80-84$ & 78 & $408 \cdot 4$ & 14 & $10 \cdot 1$ \\
$85-$ & 12 & $74 \cdot 8$ & 0 & $2 \cdot 1$ \\
All & 11483 & $49131 \cdot 0$ & 407 & $354 \cdot 1$ \\
\hline
\end{tabular}

The approximated Poisson confidence limits for the observed number of cases: $95 \%, 368 \cdot 4-448 \cdot 6 ; 99 \%, 356 \cdot 7-461 \cdot 7$.

TABLE 2 Occurrence of different malignant neoplasms in rheumatoid males

\begin{tabular}{|c|c|c|c|}
\hline Primary site & Observed & Expected & $\begin{array}{l}\text { Statistical } \\
\text { significance }\end{array}$ \\
\hline Lip, oral cavity & 12 & $13 \cdot 27$ & NS \\
\hline Esophagus & 5 & $7 \cdot 45$ & NS \\
\hline Stomach & 51 & $53 \cdot 55$ & NS \\
\hline Colon & 11 & $9 \cdot 88$ & NS \\
\hline Rectum & 7 & $10 \cdot 99$ & NS \\
\hline Liver, primary & 5 & $3 \cdot 25$ & NS \\
\hline Gallbladder & 2 & $2 \cdot 16$ & NS \\
\hline Pancreas & 16 & $14 \cdot 35$ & NS \\
\hline Respiratory organs & 171 & $132 \cdot 75$ & $\mathrm{p}<0.01$ \\
\hline Melanoma of the skin & 4 & $3 \cdot 20$ & NS \\
\hline \multicolumn{4}{|l|}{ Skin other, excluding } \\
\hline basalioma & 8 & 665 & NS \\
\hline Prostate & 30 & 30.43 & NS \\
\hline Urinary organs & 14 & 2088 & NS \\
\hline Brain & 9 & $5 \cdot 27$ & NS \\
\hline Thyroid gland & 2 & 1.44 & NS \\
\hline Hodgkin's disease & 5 & $2 \cdot 28$ & NS \\
\hline Lymphomas & 13 & $4 \cdot 84$ & $p<0.01$ \\
\hline Myeloma & 7 & $3 \cdot 26$ & $p<0.05$ \\
\hline Leukemia & 18 & $7 \cdot 10$ & $p<0.01$ \\
\hline \multicolumn{4}{|l|}{ Other and unspecified } \\
\hline sites & 17 & $21 \cdot 11$ & NS \\
\hline All & 407 & $354 \cdot 11$ & $\mathrm{p}<0.01$ \\
\hline
\end{tabular}

TABLE 3 All malignant neoplasms in females by age

\begin{tabular}{lcccr}
\hline Age & $\begin{array}{l}\text { Number of } \\
\text { rheumatoid } \\
\text { patients }\end{array}$ & $\begin{array}{l}\text { Person years } \\
\text { at risk }\end{array}$ & Observed & Expected \\
\hline $0-4$ & 97 & $130 \cdot 3$ & 0 & $0 \cdot 0$ \\
$5-9$ & 157 & $599 \cdot 3$ & 0 & $0 \cdot 1$ \\
$10-14$ & 205 & $743 \cdot 8$ & 1 & $0 \cdot 1$ \\
$15-19$ & 460 & $1336 \cdot 5$ & 0 & $0 \cdot 2$ \\
$20-24$ & 751 & $2861 \cdot 4$ & 1 & $0 \cdot 6$ \\
$25-29$ & 1079 & $4060 \cdot 4$ & 1 & $1 \cdot 2$ \\
$30-34$ & 1467 & $6085 \cdot 8$ & 4 & $3 \cdot 4$ \\
$35-39$ & 2007 & $8156 \cdot 8$ & 9 & $8 \cdot 4$ \\
$40-44$ & 2673 & $11382 \cdot 0$ & 16 & $18 \cdot 8$ \\
$45-49$ & 3481 & $15028 \cdot 9$ & 43 & $40 \cdot 7$ \\
$50-54$ & 4570 & $19083 \cdot 4$ & 58 & $64 \cdot 6$ \\
$55-59$ & 5774 & $25843 \cdot 3$ & 94 & $105 \cdot 8$ \\
$60-64$ & 5130 & $27535 \cdot 3$ & 153 & $151 \cdot 0$ \\
$65-69$ & 3260 & $20631 \cdot 5$ & 163 & $147 \cdot 5$ \\
$70-74$ & 2065 & $12123 \cdot 6$ & 114 & $116 \cdot 5$ \\
$75-79$ & 1075 & $6364 \cdot 5$ & 89 & $79 \cdot 4$ \\
$80-84$ & 290 & $2248 \cdot 3$ & 37 & $35 \cdot 2$ \\
$85-$ & 77 & $565 \cdot 5$ & 12 & $10 \cdot 5$ \\
All & 34618 & $164780 \cdot 4$ & 795 & $783 \cdot 8$ \\
\hline
\end{tabular}

The approximated Poisson confidence limits for the observed number of cases: $95 \%, 740 \cdot 7-852 \cdot 3 ; 99 \%, 724 \cdot 0-870 \cdot 3$.

TABLE 4 Occurrence of different malignant neoplasms in rheumatoid females

\begin{tabular}{lrrl}
\hline Primary site & Observed & Expected & $\begin{array}{l}\text { Statistical } \\
\text { significance }\end{array}$ \\
\hline Lip, oral cavity & 10 & $13 \cdot 32$ & NS \\
Esophagus & 21 & $20 \cdot 38$ & NS \\
Stomach & 80 & $99 \cdot 76$ & p<0.05 \\
Colon & 33 & $39 \cdot 26$ & NS \\
Rectum & 20 & $34 \cdot 47$ & p $<0.05$ \\
Liver & 6 & $5 \cdot 85$ & NS \\
Gallbladder & 10 & $14 \cdot 32$ & NS \\
Pancreas & 32 & $30 \cdot 00$ & NS \\
Respiratory organs & 34 & $30 \cdot 98$ & NS \\
Melanoma of the skin & 17 & $10 \cdot 82$ & NS \\
Skin other, excluding & & & \\
$\quad$ basalioma & 18 & $16 \cdot 58$ & NS \\
Breast & 153 & $148 \cdot 67$ & NS \\
Cervix uteri, excluding in situ & 56 & $53 \cdot 76$ & NS \\
Corpus uteri & 50 & $50 \cdot 01$ & NS \\
Ovary & 39 & $46 \cdot 83$ & NS \\
Other genital organs & 11 & $10 \cdot 82$ & NS \\
Urinary organs & 31 & $27 \cdot 79$ & NS \\
Brain & 20 & $15 \cdot 80$ & NS \\
Thyroid gland & 7 & $10 \cdot 92$ & NS \\
Hodgkin's disease & 14 & $4 \cdot 54$ & p $<0 \cdot 001$ \\
Lymphomas & 25 & $9 \cdot 34$ & p<0.001 \\
Myeloma & 21 & $9 \cdot 49$ & p<0.001 \\
Leukemia & 27 & $18 \cdot 74$ & NS \\
Other and unspecified sites & 60 & $61 \cdot 33$ & NS \\
All & 795 & $783 \cdot 78$ & NS \\
\hline
\end{tabular}

the time at which RA medication was prescribed until 31 December 1973 , or the death of the patient (a total of 213911 person years). Malignant diseases contracted before the diagnosis of RA were excluded.

The numbers of various types of neoplasms in both sexes were compared with those expected on the basis of the national cancer morbidity figures for the years $1966-1970 .{ }^{213}$ The significance of the differences was tested on the assumption that the observed number of cases followed a Poisson distribution. ${ }^{57}$ 


\section{Results}

A total of 407 neoplasms were observed in males, the expected number of cases being $354 \cdot 1$. This difference is statistically significant $(\mathrm{p}<0.01$, table 1$)$. The incidence of leukemia, lymphomas, myeloma, and cancer of the respiratory organs was significantly higher in rheumatoid patients than in the general male population (table 2 ).

In females, the incidence of malignant neoplasms was approximately equal to that expected (table 3 ). An excess of leukemia was observed, but the difference was not statistically significant. The incidence of lymphomas, myeloma and Hodgkin's disease was significantly higher in females too, while the incidence of cancer of the stomach and rectum was lower than expected (table 4).

The expected number of all cancers of the lymphatic system (leukemia, lymphomas, Hodgkin's disease, and myeloma) in both sexes was 59.6 compared with the 130 cases observed. This difference is statistically highly significant $(\mathrm{p}<0 \cdot 001)$.

\section{Discussion}

Both data registers used in this study cover the whole Finnish population. This is one major advantage of a small population ( 4.7 million). In addition, it is possible to avoid the problems facing investigators who have used hospital registers on tumors. When a study is based on hospital patient registers, it is always possible that some rheumatoid patients have malignancies that have been treated at other institutions, and the frequency of malignant neoplasms therefore seems low.

It is probable that the drug user data of the Social Insurance Institution's Population Data Register do not include all Finnish RA patients. According to this register, the prevalence of RA among the whole population in 1971 was $0.3 \%$ in males and $1.0 \%$ in females. These figures are low compared with the prevalence of RA obtained in epidemiological studies $^{148}$ which, however, usually only apply to prevalence in adult populations. On the other hand, the Finnish Cancer Registry includes practically all diagnosed cancer cases in the country. ${ }^{213}$

Cancer has been reported to be more common among patients with systemic lupus erythematosus than among, for example, those with RA and hypertension $^{126}$ or among the general population. ${ }^{29}$ The prevalence of all systemic connective tissue diseases, including SLE, in this population was only $1.7 \%$. It is probable that this does not significantly change the incidence of cancer in the total series. Moreover, no cancers were found among the causes of death of 30 SLE patients in the series of Feng t $^{\text {al }}{ }^{66}$

Many malignant neoplasms can cause rheumatic manifestations. This is especially true in children, in whom the clinical picture may suggest, e.g. juvenile rheumatoid arthritis. ${ }^{26}$ Multiple myeloma may confusingly resemble RA. ${ }^{234}$ Carcinoma polyarthritis is sometimes clinically indistinguishable from RA ${ }^{26}$ If these rheumatic manifestations preceded the overt appearance of malignancy, the patient may have been registered first as RA. Such cases are, however, rare and probably have no significant influence on the results.

In general, the incidence of epithelial cancers was as expected. The higher than expected incidence of cancer of the respiratory organs in males and the lower than expected incidence of cancer of the rectum and stomach in females may be due to chance. However, Bradley et al $^{11}$ found an association between rheumatoid factor positivity and lung cancer in a British population.

The excess risk of lymphomas, leukaemia, and myeloma in patients with RA seems indisputable. There has been discussion on whether or not continuous immunological stimulation in RA causes pron liferation and malignant transformation of some clones of immunologically competent cells. ${ }^{23}$ Hyperplasia of the lymphoid system is one of the characteristics of RA, and plasmocytosis is frequently seen in this disease. Miller ${ }^{149}$ discusses the theories on the etiologic relationship between immune diseases and malignant lymphomas. In his series of 17 patients, the onset of both processes occurred simultaneously in nine. He therefore concludes that the patients are susceptible to both diseases.

The excess risk of leukemia among RA patients was greater for males than for females. The series includes male patients with ankylosing spondylitis. This disease is strongly associated with HL-A antigen B27. Lawler et al ${ }^{121}$ found an elevated frequency of this antigen in patients with lymphoblastic leukemia. It remains to be determined whether more cases of leukemia have accumulated among patients with ankylosing spondylitis than among rheumatoids.

The low proportion of cancer deaths in rheumatoid patients ${ }^{13}{ }^{102} 152$ does not seem to be due to a low morbidity in cancer. Infections, cardiovascular diseases and renal diseases are more often the cause of death in rheumatoid than in non-rheumatoid subjects resulting in a lower than expected proportion of deaths due to cancer. ${ }^{33}{ }^{102}{ }^{152}$ In addition, the definition of the cause of death of an individual patient is always a matter of debate. 


\title{
Side-effects of azathioprine treatment in rheumatoid arthritis: analysis of 10 years of experience
}

\author{
F SPEERSTRA, A M TH BOERBOOMS, L B A VAN DE PUTTE, H J VAN \\ BEUSEKOM,M W M KRUIJSEN AND J P VANDENBROUCKE
}

From the Department of Rheumatology, St Radboud Hospital, Nijmegen, and the Department of Epidemiology, Erasmus University, Rotterdam, The Netherlands

SUMMARY Our experience with azathioprine in the treatment of rheumatoid arthritis covers ten years, during which 91 rheumatoid patients ( 66 female and 25 male) received this drug, with a median treatment period of 36 months. Total follow-up experience, during and after treatment, was 399 person years. Twelve patients died. The principal causes of death were malignant neoplasm (six patients) and cardiovascular diseases (three patients). The mortality in our patients was compared to that of the general Dutch population by the Standardised Mortality Ratio (SMR). In the male patient group a significant excess of both total mortality and mortality from malignancy was observed. The female patients showed no differences from the general population. In this follow-up study, no lymphoreticular tumours occurred during or after azathioprine therapy.

Several clinical studies have reported improvement of articular symptoms in rheumatoid patients during azathioprine therapy. ${ }^{89} 95124143177224225$ After about 15 years of experience details of treatment and management during therapy are well defined. The question whether or not the risk of malignant growth in these patients is increased is still unanswered and remains of major concern in the prescription of this drug. The present follow-up study was undertaken because of the disquieting observation of a three hundred-fold increase in occurrence of lymphoproliferative tumours, with a particular cerebral localisation, in organ transplant patients who receive azathioprine among other immunosuppressive drugs. ${ }^{174}$

\section{Patients and methods}

Ninety-one patients (66 female and 25 male) with classical or definite rheumatoid arthritis, according to the American Rheumatism Association (ARA) criteria, ${ }^{186}$ were studied retrospectively. These patients were seen at the Department of Rheumatology of the St Radboud Hospital between 1 January
1970 and 31 December 1979. All suffered from active polyarthritis for which azathioprine therapy was instituted. From these patients data were collected retrospectively in 1980-1981 on age and sex, previous antirheumatic therapy, duration of arthritis, details of azathioprine treatment, survival and eventual cause of death. These data were available on all patients. The causes of death were determined either by direct communication with the general practitioner or from our medical records or from the death certificate. The mortality experience was compared with the mortality of the general Dutch population by means of the Standardised Mortality Ratio (SMR). ${ }^{9}$ Person years of follow-up were calculated from the start of azathioprine therapy up to the common closing date of the follow-up (31 December 1979), for males and females separately, and by five-year age categories. Death rates for the calculation of the expected mortality were obtained as an average of the general population death rates in the Netherlands during the follow-up period. For each of the sexes we took the average of the five-year age category specific death rates of three calendar years in the middle of the follow-up, that is, 1973, 1974 and $1975 .{ }^{165}$ The $95 \%$ confidence limits of the SMR were calculated under the assumption that the observed and the expected are realisations of a Poisson variable ${ }^{9}$ that can be transformed to approximate normality with constant variance by square root transformation. ${ }^{19}$

\section{Results}

Characteristics of this patient population are presented in table 1. Previous drug therapy to control the rheumatic disease had consisted of gold salts $(83$ patients), D-penicillamine (35 patients) or antimalarial agents (13 patients). Four patients had been treated with another cytostatic drug; cyclophosphamide (three patients) and chlorambucil (one patient). At the start of azathioprine therapy all patients were suffering from polyarthritis and 53 
TABLE 1 Characteristics of 91 rheumatoid patients $(25$ male and 66 female) treated with azathioprine. The data are expressed as median values

\begin{tabular}{lr}
\hline Duration of disease (yr) & 9 \\
Age at start of therapy (yr) & 54 \\
Duration of treatment (mth) & 36 \\
Total dose (g) & 120 \\
Follow-up (mth) & 60 \\
\hline
\end{tabular}

were receiving corticosteroids. The median duration of the disease was nine years (range one to 33 years). The daily dosage of azathioprine ranged from 1.5 to 2 $\mathrm{mg} / \mathrm{kg}$. In most cases the drug was used without interruption. With this regimen, some patients tolerated the drug for longer than ten years.

Side-effects consisted mainly of dose related reversible bone marrow depression, but were severe enough to necessitate discontinuation of therapy in nine patients. Intractable nausea occurred in four patients. Additional reasons for discontinuing therapy were severe bacterial infections (four patients) and herpes zoster (four patients). In five other patients, azathioprine was withdrawn because appropriate outpatient clinic supervision was not guaranteed.

The clinical response was judged ineffective in seven patients after at least three months of treatment. The majority experienced clinical improvement with long-lasting remission during continuous administration of azathioprine; half of the patients were on this regimen for more than three years.

Twelve patients died during the follow-up period (table 2). Necropsy was performed in five cases. Malignancies (six patients) and cardiovascular diseases (three patients) were the most common causes of death. The observed and the expected numbers were compared using the method already described. In the male group the difference in both 'total mortality' and 'all malignancies' was statistically significant at the $5 \%$ level (tables 3 and 4). No differences were

TABLE 2 Causes of death in 12 rheumatoid patients treated with azathioprine

\begin{tabular}{llll}
\hline & Sex & Age & Necropsy \\
\hline Malignant tumours & & & \\
Bronchial carcinoma & M & 64 & + \\
& M & 60 & + \\
Renal tumour & M & 69 & + \\
Breast carcinoma & F & 69 & - \\
Cardiovascular disease & F & 57 & - \\
$\quad$ Myocardial infarction & M & 67 & - \\
& M & 51 & - \\
Infectious diseases & M & 60 & + \\
Diverticulitis & F & 56 & + \\
Pneumonia & F & 81 & - \\
Other & & & \\
Uraemia/amyloidosis & F & 66 & - \\
\hline
\end{tabular}

TABLE 3 Comparison of mortality experience ('total mortality') (ICD 000-989) in male and female rheumatoid patients treated with azathioprine, with the values expected in the general Dutch population on the basis of the Standardised Mortality Ratio (SMR)*

\begin{tabular}{llclcl}
\hline & $\begin{array}{l}\text { Follow-up } \\
\text { person } \\
\text { years }\end{array}$ & $\begin{array}{l}\text { Observed } \\
\text { number } \\
\text { of deaths }\end{array}$ & $\begin{array}{l}\text { Expected } \\
\text { number } \\
\text { of deaths }\end{array}$ & SMR & $\begin{array}{l}95 \% \text { confidence } \\
\text { limits of SMR }\end{array}$ \\
\hline Males & 102 & 7 & $1 \cdot 9$ & $3 \cdot 7$ & $1 \cdot 4-7 \cdot 0^{\dagger}$ \\
Females & 297 & 5 & $3 \cdot 1$ & $1 \cdot 6$ & $0 \cdot 4-3 \cdot 3$ \\
Total & 399 & 12 & & & \\
\hline
\end{tabular}

*Death rates for the general Dutch population were obtained from published Dutch vital statistics for the calendar years $1973,1974,1975 .{ }^{165}$

tA significant increase at the $5 \%$ level in total mortality was observed in the group of male patients.

TABLE 4 Mortality in male and female rheumatoid patients treated with azathioprine. Comparison of the malignancies observed in the deceased with the values expected for 'All malignancies' (ICD 140-209) of the Dutch population on the basis of the Standardised Mortality Ratio $(S M R)^{*}$

\begin{tabular}{lllll}
\hline & $\begin{array}{l}\text { Observed deaths } \\
\text { from malignancies }\end{array}$ & $\begin{array}{l}\text { Expected } \\
\text { number } \\
\text { of deaths }\end{array}$ & SMR & $\begin{array}{l}95 \% \text { confidence } \\
\text { limits of SMR }\end{array}$ \\
\hline Males & 4 & 0.6 & $6 \cdot 7$ & $1 \cdot 7-15 \cdot 3+$ \\
Females & 2 & $0 \cdot 9$ & $2 \cdot 1$ & $0 \cdot 2-6 \cdot 2$ \\
Total & 6 & & & \\
\hline
\end{tabular}

*Death rates for the general Dutch population were obtained from published vital statistics for the calendar years $1973,1974,1975 .^{165}$

$\dagger$ The difference in the group of male patients is statistically significant at the $5 \%$ level.

found in the female group. In four male patients squamous cell bronchial carcinoma was diagnosed. Since malignant tumours of the respiratory tract, as well as cardiovascular disease, were the only causes of death in the male patients, we reviewed the patients' smoking habits from our medical records. An overwhelming majority (96\%) had a history of cigarette smoking, compared to $55 \%$ in the general male Dutch population. ${ }^{227}$

In the interpretation of our results it should be noted that one female patient had strong clinical evidence of malignant renal neoplasm (haematuria with massive tumour on intravenous pyelography), but necropsy was not performed. She was classified as a cancer death.

No differences with respect to total dose and duration of azathioprine therapy could be demonstrated between the patients who developed malignancies and those who did not. Seventy-nine patients are still alive and under medical outpatient care, which includes regular physical examination, blood counts, analysis of liver function and urine testing. $X$-rays of the chest and, if indicated, contrast radiological examinations have been performed. At the end of the follow-up period, no evidence of further malignancies had occurred in these patients, and no lymphoproliferative neoplasms have been diagnosed during or after azathioprine therapy. 


\section{Discussion}

For several years the clinical efficacy of azathioprine in the treatment of chronic inflammatory diseases, including rheumatoid arthritis, has been firmly established. Studies in rheumatoid patients, both in controlled $^{124143224225}$ and in open studies, ${ }^{89} 95177$ have revealed improvement of articular symptoms in the majority of patients. Moreover, reduction of corticosteroid dosage has been achieved in the first year of treatment. ${ }^{143}{ }^{177}$ Patients included in these studies were all suffering from long-standing active polyarthritis despite previous antirheumatic therapy.

In our study the majority of patients had previously been treated with either gold salts or D-penicillamine. Disease activity was reflected in the number of patients receiving corticosteroids $(58 \%)$. In accordance with earlier observations ${ }^{124} 143224$ improvement of articular symptoms was most pronounced in the first six months of treatment with azathioprine and could be maintained, to some degree, during long-term treatment with a dose of 1.5 to $2.0 \mathrm{mg} / \mathrm{kg}$ daily. Substantial reduction of corticosteroids (50\% or more) was effected in patients who tolerated the drug for at least six months.

Since long-term treatment is anticipated in rheumatoid patients, one might suspect that the cytotoxic and immunosuppressive properties of azathioprine may increase the risk of oncogenesis. Cytotoxic effects on bone marrow ${ }^{105}$ and the urogenital tract, ${ }^{1}$ as well as an increased number of chromosomal aberrations, have been reported ${ }^{95}$ but could not be confirmed by others. ${ }^{230}$ Whether a measurable degree of immunosuppression is achieved by low to moderate dosage regimens is uncertain. ${ }^{24} 208$ The high incidence of lymphomas, especially reticulum cell sarcoma, with intracerebral localisation, in patients with organ transplants is unprecedented. ${ }^{15}{ }^{174}$ Among other considerations, this fact focuses attention on the possible oncogenic effect of azathioprine in this category of patient. Nevertheless, the literature does not clearly show whether the occurrence of any particular malignancy in rheumatoid patients is increased in association with azathioprine therapy.

Two studies report a higher incidence of lymphomas, but both series included various chronic inflammatory conditions $\mathrm{s}^{115} 174$ and different cytostatic drugs were considered in one. ${ }^{174}$ Moreover, the risk of development of this type of tumour seems to be increased in rheumatoid patients ${ }^{101} 124$ and this fact should be taken into account.

Case reports dealing with the presumed oncogenic effect of azathioprine include leukaemia, ${ }^{32} 79$ adenocarcinoma of the lung ${ }^{177}$ and breast carcinoma ${ }^{177}$ Two cases are of special interest, because these patients developed malignant lymphoma with intracerebral localisation in both. ${ }^{127}{ }^{158}$ Our observations, covering 399 person years, revealed no lymphoreticular malignancies during or after azathioprine therapy. The excess mortality in the 25 male patients attracts attention, especially because four patients developed bronchial carcinoma.

In rheumatoid arthritis, death rates tend to be higher in the younger patients. ${ }^{3355125} 219$ Principal causes of death are cardiovascular disease, renal failure $^{102} 156$ and, in earlier studies, infectious diseases. ${ }^{335}$ Two authors reported an increase of malignancies in their series, ${ }^{125}{ }^{151}$ but found no difference in the type of tumour between the cytostatic treated group and the control patients. ${ }^{64125}$ The increased number of squamous cell bronchial carcinomas found in our male patients may well be related to their smoking habits. Nevertheless, careful observation and follow-up of patients with rheumatoid arthritis receiving azathioprine should continue. Meanwhile this drug should only be given to those patients with severe rheumatoid arthritis who do not respond to, or cannot tolerate, other antirheumatic drugs. 


\title{
Compliance and long-term effect of azathioprine in 65 rheumatoid arthritis cases
}

\author{
P. VAN WANGHE AND J DEQUEKER \\ From the Afdeling Reumatologie, Academisch Ziekenhuis, B-3000 Leuven, Belgium
}

SUMmary Azathioprine has been used in our unit as a third line disease modifying drug (DMD) since 1969. In 65 patients with severe rheumatoid arthritis (RA), [45 females and 20 males, mean age $55 \cdot 2$ years (32 to 76 ), mean duration of disease 14 years (1 to 41)], azathioprine was given in an average dose of 1.5 $\mathrm{mg} / \mathrm{kg}$ body weight/day for a mean duration of 33.4 months (range 1 to 108 ).

The mean follow-up was five years. One hundred and eighty-four patient years of treatment with azathioprine were observed. After three months' treatment, significant subjective and objective improvement was observed in $65 \%$ of the cases. This improvement remained in 29 cases who received continuous treatment for two years. In 12 of the 20 seropositive RA cases, a reduction of at least three dilutions in the rheumatoid factor titre was noted. In the 24 patients who were corticosteroid dependent, the dosage of steroids could be reduced by $35 \%$ and in four steroids could be stopped completely. Compliance after two years $(n=54)$ was still $67 \%$. Azathioprine treatment had to be stopped in 23 patients because of ineffectiveness in nine and adverse effects in 14 . In three cases (4.6\%) a malignant tumour occurred: one lymphoma and two adenocarcinomata. Low dose azathioprine therapy was shown to be useful as a third line disease modifying drug in RA without an increase in oncogenic risk. Compliance for azathioprine was found to be very satisfactory compared to other drugs.

Since the introduction in $1951^{106}$ of immunosuppressants in the treatment of rheumatoid arthritis, several studies have shown that immunosuppressive drugs can induce a measurable improvement in the inflammatory process. ${ }^{183438566995109113177214225237}$ Although the mechanism of action is still unclear, these agents have been used extensively in recent years. In our unit, azathioprine has been in use since 1969 , and is considered as the third line 'disease modifying drug' after gold salts and D-penicillamine. In this retrospective study, the long-term effect, compliance and adverse effects of azathioprine have been evaluated in 65 patients who received the drug in the past decade.

\section{Patients and methods}

Sixty-five patients with classical rheumatoid arthritis, according to the American Rheumatism Association (ARA) criteria, were entered into the study. The characteristics of the patients are shown in table 1 .

There were 20 men and 45 women, with ages ranging from 32 to 76 years (mean 55.2 years), and the average duration of rheumatoid arthritis was 14 years (range 1 to 41 years). The mean starting dosage of azathioprine was $1.5 \mathrm{mg} / \mathrm{kg} /$ day. Dosage was $\Omega$ adjusted during the course of the disease according to clinical response. This study covers 184 patient treatment years, with an average duration of $33.4 \%$ months (range 1 to 108) of azathioprine therapy.

Treatment before azathioprine is listed in table 2 . Most of the patients had had a course of gold, antimalarials or D-penicillamine treatment before azathioprine was started. Gold, antimalarials or D-penicillamine were discontinued before starting azathioprine. Fewer patients were treated with D-penicillamine since this drug was introduced several years after azathioprine in our unit. In patients receiving steroids and/or depot-ACTH, an attempt was made to reduce the dose as much as possible.

TABLE 1 Characteristics of 65 patients treated with azathioprine

\begin{tabular}{ll}
\hline Male/female & $20 / 45$ \\
Mean age & $55 \cdot 2 \mathrm{yr}(32-76)$ \\
Seronegative/seropositive & $17 / 48$ \\
Mean duration of disease & $14 \mathrm{yr}(1-41)$ \\
Mean dosage of azathioprine & $1.5 \mathrm{mg} / \mathrm{kg} /$ day \\
Mean duration of therapy & $33.4 \mathrm{mth}(1-108)$ \\
Mean duration of follow-up & $5 \mathrm{yr}$ \\
Total patient treatment years & 184 \\
\hline
\end{tabular}

TABLE 2 Therapy before azathioprine

\begin{tabular}{lr}
\hline Gold salts & 41 \\
Antimalarials & 23 \\
D-penicillamine & 15 \\
Prednisone & 8 \\
Depot-ACTH & 12 \\
Prednisone + ACTH & 4 \\
\hline
\end{tabular}


All patients continued the treatment with salicylates or non-steroidal anti-inflammatory drugs (NSAID), which they were taking when azathioprine was started.

The indications for azathioprine therapy are shown in table 3. Azathioprine was started, in most cases, because of failure of, or adverse effects to, 'classic therapy': gold, chloroquine or D-penicillamine. In 14 patients treatment was given in order to reduce corticosteroid dependency, and in six to control systemic involvement such as pericarditis or vasculitis. Each patient was seen at regular intervals at the outpatient clinic where the following assessments were made: subjective status (better, unchanged, worse), morning stiffness (hours) and clinical examination with special attention to the joint status. Laboratory tests performed at each visit included blood counts, sedimentation rate (ESR), C-reactive protein (CRP), rheumatoid factor, serum protein electrophoresis, liver function tests and urine analysis. The activity of the rheumatoid arthritis, as described in the charts, was defined by the following clinical activity scores, which were designed for retrospective studies. ${ }^{205}$

Grade 0 (remission)-no morning stiffness, no persistent joint pain, tenderness or swelling.

Grade I (mild RA)-persistent joint pain without morning stiffness, joint tenderness or swelling.

Grade II (moderately active RA)-presence of

TABLE 3 Indications for azathioprine therapy

\begin{tabular}{lr} 
Failure of 'classic' therapy & 30 \\
Adverse reactions to 'classic' therapy & 15 \\
Corticosteroid dependency & 14 \\
Systemic involvement & 6 \\
\hline
\end{tabular}

joint pain, tenderness and swelling and morning stiffness $<120$ minutes.

Grade III (severe RA)-persistent moderate or marked articular tenderness and swelling, associated with warmth and/or erythema and morning stiffness $>120$ minutes.

When charts were incomplete because of failure to attend during the last year, or death, information on the patient's condition, or cause of death, was sought from the family physician.

\section{Results}

\section{THERAPEUTIC EFFECTS}

After three months of therapy $42(65 \%)$ of the 65 patients had improved subjectively, morning stiffness had decreased in $42(65 \%)$ and the number of swollen joints had reduced in $46(70 \%)$.

To evaluate the long-term effect, a group of 29 patients who were treated for at least 24 months were selected for study. The results for this group who tolerated the drug are shown in table 4 . Subjective improvement occurred in $21(72 \%)$ after three months. This effect lasted for 24 months and was confirmed by improvement in the clinical findings; morning stiffness, number of swollen joints and ESR decreased significantly. The titre of the latex test decreased by three or more tube dilutions in 12 of 20 seropositive patients after 24 months. There was no significant change in haemoglobin levels, CRP or albumin/globulin ratio.

In 24 cases who were dependent on corticosteroids, it was possible to reduce the dosage by approximately $35 \%$, and in four steroids could be stopped completely. The response of systemic involvement (two vasculitis, two pericarditis, one

TABLE 4 Clinical outcome of 29 RA patients receiving azathioprine for 24 months

\begin{tabular}{|c|c|c|c|c|c|}
\hline & 0 months & 3 months & 6 months & 12 months & 24 months \\
\hline \multicolumn{6}{|l|}{${ }^{*}$ Subjective } \\
\hline Improvement & - & 72 & 71 & 75 & 72 \\
\hline Unchanged & - & 20 & 17 & 21 & 16 \\
\hline Worse & - & 8 & 12 & 4 & 12 \\
\hline \multicolumn{6}{|c|}{ *Clinical activity grade } \\
\hline 0 & 0 & 0 & 4 & 11 & 11 \\
\hline I & 13 & 30 & 46 & 30 & 39 \\
\hline II & 45 & 52 & 50 & 59 & 39 \\
\hline III & 42 & 18 & 0 & 0 & 11 \\
\hline \multicolumn{6}{|c|}{ Morning stiffness (hours) } \\
\hline $\begin{array}{l}\text { Mean } \pm \text { SD } \\
\mathrm{p}^{+}\end{array}$ & $2 \cdot 3 \pm 1 \cdot 3$ & $\begin{array}{l}1.6 \pm 1 \\
p<0.05\end{array}$ & $\begin{array}{l}17 \pm 12 \\
\text { NS }\end{array}$ & $\begin{array}{l}1 \pm 1 \\
p<0.001\end{array}$ & $\begin{array}{l}1 \cdot 3 \pm 1 \cdot 2 \\
p<0.01\end{array}$ \\
\hline \multicolumn{6}{|c|}{ No of swollen joints } \\
\hline $\begin{array}{l}\text { Mean } \pm S D \\
\mathrm{p}^{\dagger}\end{array}$ & $6 \cdot 3 \pm 3 \cdot 5$ & $\begin{array}{l}4.5 \pm 2.8 \\
p<0.02\end{array}$ & $\begin{array}{l}3.6 \pm 2.9 \\
p<0.01\end{array}$ & $\begin{array}{l}4 \pm 3 \\
p<0.02\end{array}$ & $\begin{array}{l}3 \pm 2 \cdot 1 \\
\mathrm{p}<0.01\end{array}$ \\
\hline \multicolumn{6}{|l|}{ ESR } \\
\hline $\begin{array}{l}\text { Mean } \pm \text { SD } \\
\mathrm{p}^{+}\end{array}$ & $69 \pm 33 \cdot 3$ & $\begin{array}{l}53 \pm 23.6 \\
p<0.001\end{array}$ & $\begin{array}{l}50 \pm 19.6 \\
p<0.01\end{array}$ & $\begin{array}{l}52 \pm 28 \cdot 7 \\
p<0.01\end{array}$ & $\begin{array}{l}40 \pm 24.4 \\
p<0.001\end{array}$ \\
\hline
\end{tabular}

* Percentage of patients assessed.

+Compared to the initial value using the paired Student's $t$ test. 
pleuritis, one Felty's syndrome) was good in all six cases.

\section{COMPLIAN C E}

In order to assess the long-term effects and tolerance of azathioprine, compliance to treatment with azathioprine was evaluated in the 54 patients who started their treatment two years before completion of this study. A compliance study may give important information on therapeutic benefit since a drug which does not adequately relieve pain, or which causes intolerable side-effects, will be discarded by the patient. After two years, $36(67 \%)$ patients were still continuing azathioprine therapy.

The figure compares the drop-out curve presented as life table survival curve for azathioprine with those of gold, D-penicillamine, hydroxychloroquine and levamisole, as published by Husain and Runge. ${ }^{96}$ Compared to the compliance for other disease modifying drugs, azathioprine scores very well.

\section{WITHDRAWALS AND ADVERSE EFFECTS}

Twenty-three $(35 \%)$ of the 65 patients were withdrawn, nine because of inadequate therapeutic response and 14 because of untoward effects which were: gastrointestinal disturbance in six cases, bone marrow suppression (leucocytes $<3500$, thrombocytes $<10^{5} / \mathrm{mm}^{3}$ ) in seven cases and liver function disturbance in one case.

Thirty-eight $(58 \%)$ of our patients experienced side-effects mainly in the initial three months of therapy (table 5).

In three cases a malignant tumour occurred: an undifferentiated lymphoma of the stomach 3.5 years

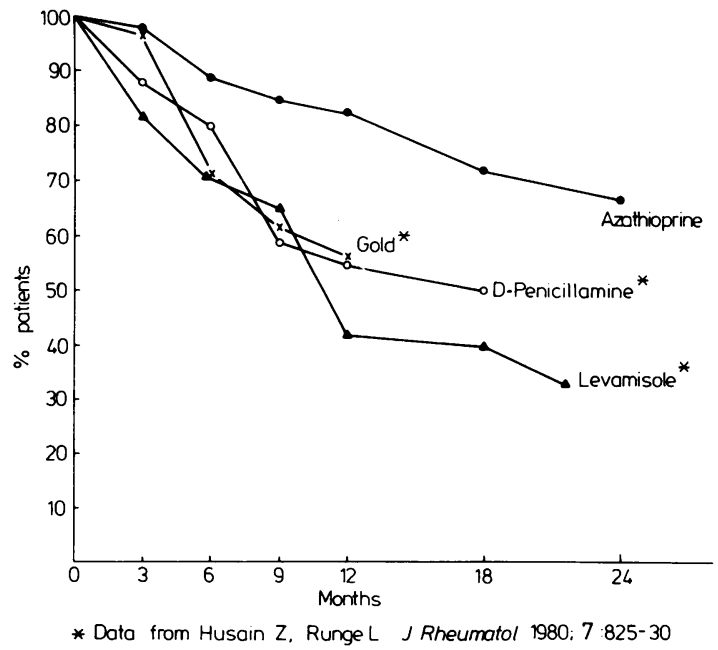

FIGURE Patient compliance: disease modifying drugs (DMD).
TABLE 5 Untoward events in 38 of 65 patients on azathioprine

\begin{tabular}{lrl}
\hline GI disturbance & 16 & $(25 \%)$ \\
Bone marrow suppression & 16 & $(25 \%)$ \\
Infections & 6 & $(9 \%)$ \\
Stomatitis & 3 & $(5 \%)$ \\
Haematuria & 6 & $(9 \%)$ \\
Skin rash & 2 & $(3 \%)$ \\
Liver function disturbance & 2 & $(3 \%)$ \\
\hline
\end{tabular}

after start of treatment in a 54-year-old man with Felty's syndrome (the patient is still alive and well seven years later); one adenocarcinoma of the stomach in a 65-year-old female 6.5 years after stopping 1.5 years' treatment with azathioprine (patient is still alive and well ten years later); and one cystadenocarcinoma of the appendix in a 66-year-old female two years after stopping seven years of azathioprine treatment.

Sixteen patients died and the causes of death are shown in table 6. The average age of death was 64.8 years (range 52 to 73 ).

\section{Discussion}

The results of our study are in accordance with those of others. ${ }^{1835695109177225}$ After three months of azathioprine treatment, with an average starting dose of $1.5 \mathrm{mg} / \mathrm{kg} /$ day, the majority of our patients, unresponsive to conventional therapy, showed evidence of improvement by subjective, clinical and laboratory criteria. In a group of patients who

TABLE 6 Cause and age of death of 16 patients who died during follow-up period

\begin{tabular}{|c|c|c|c|c|c|}
\hline No & Age & Sex & Cause of death & $\begin{array}{l}\text { Duration of } \\
\text { therapy } \\
(y r)\end{array}$ & $\begin{array}{l}\text { Interval between } \\
\text { termination of } \\
\text { therapy and } \\
\text { death }(y r)\end{array}$ \\
\hline \multirow{5}{*}{4} & 69 & $\mathbf{M}$ & $\begin{array}{l}\text { Postoperative } \\
\text { complication }\end{array}$ & 5 & 2 \\
\hline & 60 & $\mathbf{M}$ & $\begin{array}{l}\text { Postoperative } \\
\text { complication }\end{array}$ & 9 & 0 \\
\hline & 67 & $\mathbf{M}$ & $\begin{array}{l}\text { Postoperative } \\
\text { complication }\end{array}$ & 32 & 0 \\
\hline & 64 & $\mathrm{~F}$ & $\begin{array}{l}\text { Postoperative } \\
\text { complication }\end{array}$ & 45 & 45 \\
\hline & 71 & $\mathbf{F}$ & $\begin{array}{l}\text { Cardiovascular } \\
\text { disease }\end{array}$ & 0.75 & 25 \\
\hline \multirow[t]{3}{*}{4} & 69 & $\mathbf{F}$ & $\begin{array}{l}\text { Cardiovascular } \\
\text { disease }\end{array}$ & 15 & 6 \\
\hline & 67 & $\mathbf{M}$ & $\begin{array}{l}\text { Cardiovascular } \\
\text { disease }\end{array}$ & 6.5 & 0 \\
\hline & 64 & $\mathbf{F}$ & $\begin{array}{l}\text { Cardiovascular } \\
\text { disease }\end{array}$ & 3 & 0 \\
\hline \multirow{2}{*}{2} & 73 & $\mathbf{F}$ & Cachexia & $4 \cdot 5$ & 0 \\
\hline & 63 & $\mathbf{M}$ & Cachexia & 1 & 0 \\
\hline \multirow{2}{*}{2} & 68 & $\mathbf{F}$ & Sepsis & $2 \cdot 5$ & 0 \\
\hline & 76 & $\mathrm{~F}$ & Sepsis & 3 & 7 \\
\hline 1 & 64 & $\mathbf{F}$ & Malignancy & 7 & 2 \\
\hline 1 & 64 & $\mathbf{F}$ & Diabetes & 3 & 0 \\
\hline 1 & 52 & $\mathbf{M}$ & Suicide & $3 \cdot 5$ & 0 \\
\hline 1 & & $\mathrm{~F}$ & Unknown & 05 & - \\
\hline
\end{tabular}


tolerated the drug for a long period, this improvement continued for at least two years. Except for sedimentation rate and rheumatoid factor, no important changes in biochemical parameters of inflammation were observed.

A moderate corticosteroid sparing effect was also observed. The clinical response observed in this retrospective study is similar to other studies. Urowitz et $a^{225}$ treated their patients for 24 weeks and found that, after 12 and 24 weeks, a significant clinical improvement occurred in more than $80 \%$ of their patients, independent of the dose of the drug taken. Laboratory evaluation revealed no important changes. Hunter $e^{t} a^{95}$ followed their patients up to 40 months; the initial improvement at 16 months continued until 40 months. Biochemically they found a decrease in the latex titre, ESR and immunoglobulin level. Pinals ${ }^{177}$ treated his patients for at least 20 weeks and observed clinical improvement in $56 \%$ of his cases. Sedimentation rate did not change significantly, but the dose of corticosteroids could be reduced in seven of the 17 patients.

Dwosh et $a^{56}$ compared azathioprine with gold and chloroquine and found significant improvement in all three groups after 12 and 24 weeks. The latex titre fell significantly in the azathioprine and gold treated groups, but ESR rose in the azathioprine group after 12 weeks' treatment, despite continued clinical improvement. Berry et $a^{18}{ }^{18}$ compared azathioprine with D-penicillamine in a one-year study and found equal efficacy of both drugs (70\%). ESR and latex titre fell in both groups. Currey $e^{2} a^{38}$ compared azathioprine with gold and cyclophosphamide and observed a comparable improvement in the three treatment regimens. The ESR fell in the three groups, rheumatoid factor did not change and steroid requirements tended to fall.

The higher 'compliance' with azathioprine compared to other disease modifying drugs ${ }^{96}$ may be explained by three facts: as a measure of efficacy it is in accordance with the observed therapeutic response; since it is a toxic drug, the follow-up of patients is generally more intensive, which has a positive effect on patients' motivation; patients know that azathioprine is, more or less, the 'last chance' drug in controlling their disease. Nevertheless, $35 \%$ of our patients were withdrawn from azathioprine therapy, this being higher than the 18 to $32 \%$ reported in the literature. Adverse reactions were frequent $(58 \%)$, but led to discontinuation of treatment in only $21 \%$. Most important were gastrointestinal disturbance and bone marrow suppression, the latter being reversible in all cases. The frequency of untoward events in our study is comparable with the mean frequency in the studies mentioned above. ${ }^{18385695109177225}$ Only haematuria, observed in six cases, has never been associated with azathioprine and is probably the result of other factors (NSAID, infection, cyclophosphamide).

Malignancy occurred in three patients $(4 \cdot 6 \%)$. Pinals ${ }^{177}$ reported an incidence of $8 \%$ and Lewis $e t$ $a l^{125} 4 \%$, which was lower than in patients not treated with immunosuppressants $(10 \%)$, suggesting that an altered immune status of $\mathrm{RA}$ patients ${ }^{81}$ might facilitate oncogenesis. Immunosuppressants may play a role in tumour induction by chromosomal damage $^{95}$ or other mechanisms. According to published reports, alkylating agents are much more suspect than antimetabolites. ${ }^{108} 154184$

In our study 16 patients died during follow-up, mainly because of cardiovascular disease and postoperative complications (table 6). We assume that the high mortality rate is the result of the lower life expectancy of rheumatoid arthritis patients of this age group (52 to 73 years, mean 64.8 years). ${ }^{33102125182} 219$ In only two cases is it possible that there was a causative relation between treatment and death (that is, infection and neoplasia).

One can confirm that azathioprine has an important antirheumatic activity but, considering its toxicity and potential oncogenic risk, its use should be reserved for the specific indications of:

(1) failure of conventional therapy in active rheumatoid arthritis;

(2) 'malignant' forms of rheumatoid arthritis; and

(3) corticosteroid dependency. 


\title{
Rheumatoid arthritis: treatment with azathioprine (IMURAN (R)). Clinical side-effects and laboratory abnormalities
}

\author{
J K WHISNANT AND J PELKEY \\ From the Immunology Section, Department of Clinical Investigation, Burroughs Wellcome Co, USA
}

SUMMARY A retrospective review of the literature has been carried out to determine laboratory abnormalities occurring in patients with rheumatoid arthritis (RA) treated with azathioprine, in order to establish a profile for this agent in the treatment of this disease.

A total of 542 patients in 24 studies, reported in the literature, were given a range of doses of azathioprine for up to four years. Fifteen percent of patients were withdrawn because of toxicity. The two major toxic effects were gastrointestinal symptoms and alteration in blood counts. Clinically significant leucopenia (less than $2500 / \mathrm{mm}^{3}$ ) occurred in 14 of the total of 93 patients reported to have developed leucopenia. Some adverse reactions, which would have been expected from the use of azathioprine in other diseases, were uncommon, namely significant infections, hepatotoxicity and pancreatitis. Adverse experience with azathioprine in rheumatoid arthritis compares well with other slow-acting, or disease modifying, drugs.

Immunosuppressive therapy of rheumatoid arthritis (RA) with the thiopurine azathioprine (IMURAN (R)) has been reported since 1965 . The initial experiences with this drug stimulated some enthusiasm and there were 11 publications in the period 1965-1970. 1522455172129143150206207212 In the next five years (1971-1975), the problems of significant haematological toxicity and the possibility of malignancy in renal transplantation patients treated with azathioprine caused concern, so that less investigation and interest was evident during these years. Major studies were reported again in 1976-1977 and some of these, especially those by Balken, ${ }^{12}$ Berry et al, ${ }^{18}$ Dwosh et al ${ }^{56}$ and Goebel et $a l,{ }^{80}$ stimulated re-evaluation of the place of azathioprine in the treatment of rheumatoid arthritis. A total of 24 studies published in the English litera- ture, or available in translation, over an 11-year period is now available for review to assess the composite adverse experience with azathioprine in this non-transplant disease category.

\section{Methods of review}

A composite azathioprine literature listing, including computerised reference banks, was first used to establish a primary literature data base. There are, as always, multiple citations which mention azathioprine for RA but which give no clinical data or patient

TABLE 1 Twenty-four articles reviewed with year of publication, number of patients treated with azathioprine and concomitant therapy

\begin{tabular}{|c|c|c|c|}
\hline Reference & Year & $\begin{array}{l}\text { Azathio- } \\
\text { prine }\end{array}$ & Other therapy \\
\hline Apostoloff $e t a^{7}$ & 1974 & 9 & \\
\hline Balken $^{12}$ & 1976 & 41 & $\begin{array}{l}\text { Steroids, analgesics, } \\
\text { antirheumatic agents }\end{array}$ \\
\hline Barnikol and Vorlaender ${ }^{15}$ & 1967 & 9 & Antirheumatic \\
\hline${ }^{*}$ Berry et al ${ }^{18}$ & 1976 & 33 & $\begin{array}{l}\text { Steroids, anti- } \\
\text { inflammatory agents }\end{array}$ \\
\hline Bruckner et $a l^{22}$ & 1969 & 6 & Steroids \\
\hline${ }^{*}$ Cade $e t a l^{25}$ & 1976 & 18 & Salicylates, steroids (1) \\
\hline${ }^{*}$ Currey et $a l^{38}$ & 1974 & 44 & $\begin{array}{l}\text { Corticosteroids, aspirin, } \\
\text { paracetamol }\end{array}$ \\
\hline Denman et $a l^{45}$ & 1970 & 5 & Prednisone \\
\hline${ }^{*}$ Dixon $e t a l^{49}$ & 1971 & 14 & \\
\hline Dodson and Bennett ${ }^{51}$ & 1969 & 23 & \\
\hline${ }^{*}$ Dwosh et $a l^{56}$ & 1977 & 11 & Steroids (1) \\
\hline Fricke and Deicher ${ }^{72}$ & 1969 & 8 & $\begin{array}{l}\text { 6-methyl-prednisolone, } \\
\text { phenylbutazone, } \\
\text { indomethacin }\end{array}$ \\
\hline${ }^{*}$ Goebel et $a l^{80}$ & 1976 & 34 & Aspirin, indomethacin \\
\hline Khanna and Woodbury ${ }^{114}$ & 1973 & 21 & \\
\hline Levy et $a^{123}$ & 1975 & 49 & Salicylates, steroids \\
\hline Lorenzen et al ${ }^{129}$ & 1969 & 9 & \\
\hline Mason et al ${ }^{143}$ & 1969 & 27 & Steroids, paracetamol \\
\hline Moens and Brocteur ${ }^{150}$ & 1965 & 11 & $\begin{array}{l}\text { Steroids, 6-methyl- } \\
\text { deltahydrocortisone, } \\
\text { actinomycin-C }\end{array}$ \\
\hline Pinals $^{177}$ & 1976 & 21 & Corticosteroids (17) \\
\hline Swannell and Coomes ${ }^{206}$ & 1969 & 9 & Steroids (9), ACTH (1) \\
\hline Swannell and Kersley ${ }^{207}$ & 1969 & 26 & Prednisolone \\
\hline Tausch et $a l^{212}$ & 1970 & 73 & Steroids (46) \\
\hline${ }^{*}$ Urowitz et al ${ }^{224}$ & 1973 & 17 & Salicylates \\
\hline *Urowitz et al ${ }^{22,5}$ & 1974 & 24 & Salicylates \\
\hline
\end{tabular}

*Controlled study. 
experiences, which were eliminated. A smaller, but significant, number of publications report fewer than five patients without comparison or control patient group information; these, too, were eliminated. The selected published studies included in this analysis are listed in table 1, with the numbers of patients and year of each publication. Some of the 24 studies were published more than once, but duplicate reporting of the same patient groups was consolidated in abstracting information. The authors' statements regarding diagnosis, or diagnostic criteria, were accepted. Patients included in these azathioprine studies were usually those with severe, disabling arthritis, unresponsive to conventional therapy. Careful attention was directed to eliminating the patients with diseases other than RA sometimes included in these reports.

All clinical toxicities and laboratory abnormalities included in each publication were tabulated. Summary profile sheets on each study were prepared, and these are available from the authors. It was not possible to impose a uniform grading system for severity of toxicities, except where specific haematological or other laboratory data were given. Results are given in terms of total percentage of patients with a specific toxicity. Particular attention was given to cases requiring withdrawal from treatment, to cases with life-threatening or fatal reactions and to toxicities occurring in control groups, where such were included (designated by asterisk in table 1). A detailed review of original patient records was conducted on four of the 24 studies: Urowitz et al, ${ }^{224}$ Urowitz et al ${ }^{225}$ Levy et al ${ }^{123}$ and Cade et al.$^{25}$

\section{Results}

From this review of the literature, 542 patients could be studied (table 1). The most important side-effect of this antimetabolite thiopurine is its effect on white cell production manifest by peripheral leucopenia. The total incidence of haematological toxicity is illustrated in fig 1 . Individual patient side-effects may have been multiple, and the percentage represented is the total for that individual toxicity. There were 142 haematological toxicity events reported in a total of 542 patients. Had all of these events occurred in separate patients, a total of $26 \%$ of patients might have been affected.

Leucopenia of any degree was mentioned in 93 $(17 \%)$ of the 542 patients. Of these 93 patients, actual leucocyte values were reported in 67; these are summarised in fig 1 . Fourteen patients were reported to have a leucopenia low enough to cause clinical risk
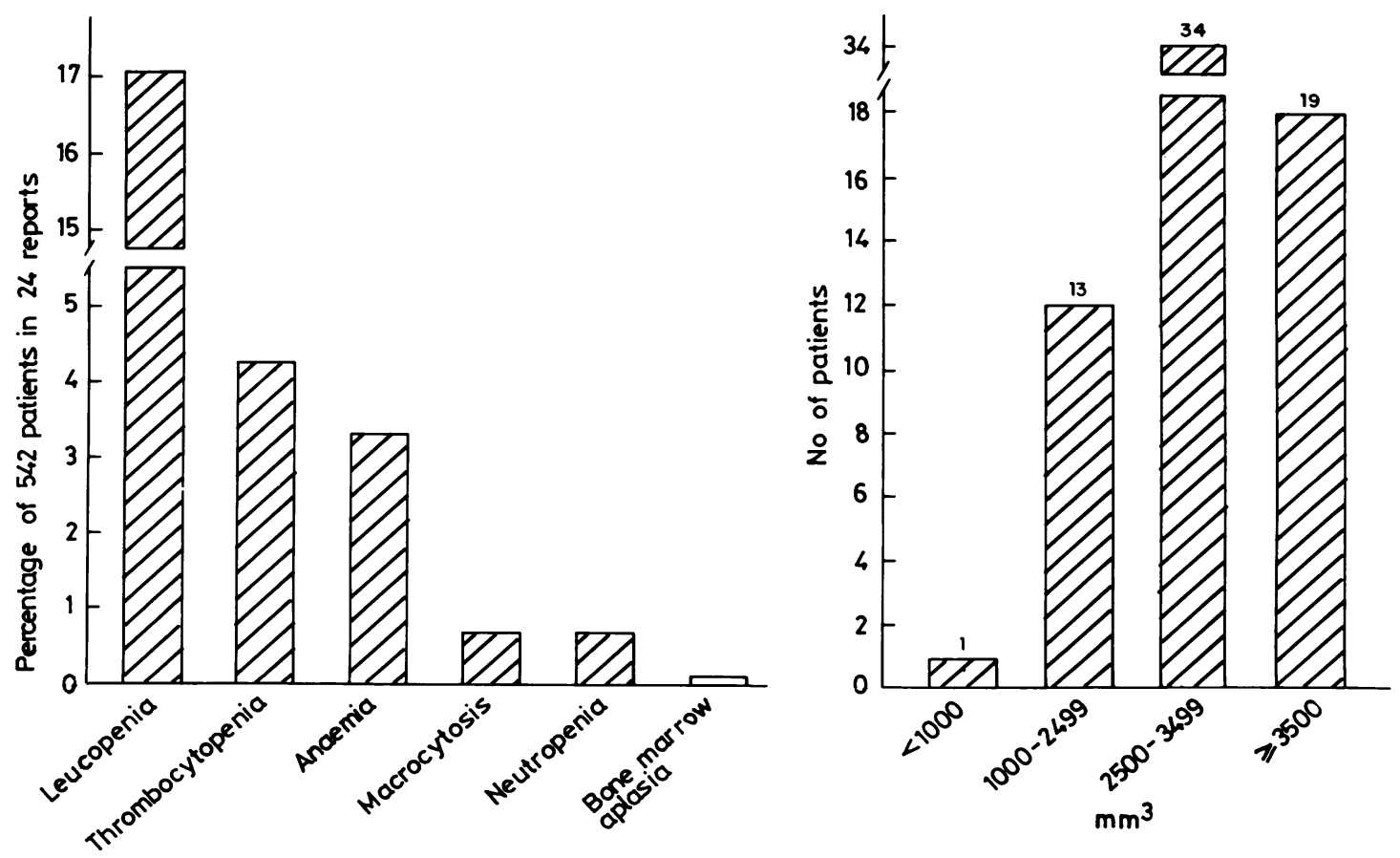

FIG 1 Incidence of haematological adverse effects reported in 24 published reports of 542 patients receiving azathioprine (left). No of cases with degree of leucopenia as reported in patients on whom data are available (right). 
(less than $2500 / \mathrm{mm}^{3}$ ). The majority of patients had white blood cell counts between 2500 and $3500 / \mathrm{mm}^{3}$, and an additional approximately $25 \%$ had counts above $3500 / \mathrm{mm}^{3}$. There were an additional four cases mentioned to have developed 'neutropenia', but the degree of neutropenia was not specified. Discontinuation of therapy or withdrawal from a study was uncommon as a result of haematological toxicity.

A review of the six studies published in 1976-1977 12 18255680177 shows an incidence of leucopenia of only $10 \%$.

The haematological data from 108 patients in the four studies reviewed in depth were similar to those

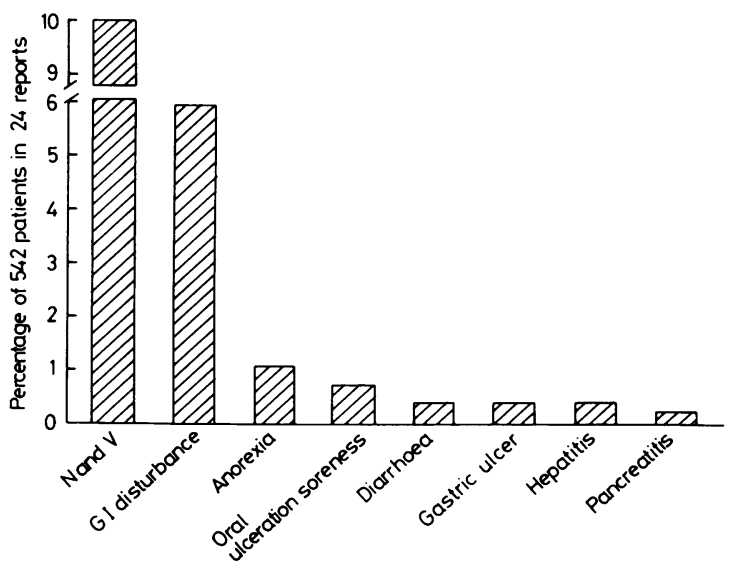

FIG 2 Incidence of gastrointestinal adverse effects reported in 24 published reports of 542 patients receiving azathioprine. tabulated from the literature. The occurrence of multiple blood count abnormalities in one patient was confirmed in these reviews. The study by Levy et al, ${ }^{123}$ in which a higher mean dose of azathioprine $(2.9$ $\mathrm{mg} / \mathrm{kg} /$ day) was used, included a relatively high incidence of leucopenia. A patient's disease activity measurements did not correlate with the degree of leucopenia.

Gastrointestinal complaints were reported 103 times in the 542 patients, an incidence of $19 \%$ (fig 2 ). Approximately $10 \%$ of patients had nausea and /or vomiting, and gastrointestinal symptoms, not otherwise specified, were reported in $6 \%$ of patients. Other symptomatology was reported in less than $1 \%$ of the total for each of the following: anorexia six cases, ulceration four cases, diarrhoea two cases and ulcer two cases. Two gastrointestinal toxicities, reported in other investigational uses of azathioprine, were not found in a significant percentage in this review. There were only two reports of hepatotoxicity, a significant problem in the use of azathioprine in renal transplantation. There was only one report of idiosyncratic, or 'allergic', pancreatitis which has been reported in inflammatory bowel disease treated with azathioprine.

The review paid particular attention to patient withdrawal and to prohibitive or life-threatening reactions reported by the authors. Interruption of azathioprine treatment, or withdrawal of patients, was mentioned in relation to both haematological and gastrointestinal toxicity. Eighty-one patients were withdrawn because of drug-related adverse reactions $(15 \%)$.

Reports of infection $(2.4 \%)$ in the context of this immunosuppressive therapy were lower than might have been expected, with most of these being bacterial infection of a non-threatening nature. Five patients, in three reports published before 1970, died

TABLE 2 Deaths or neoplasia reported in seven patients

\begin{tabular}{|c|c|c|c|c|c|c|c|}
\hline Reference & Diagnosis & Sex & Age & $\begin{array}{l}\text { Azathioprine } \\
\text { (mg/day) }\end{array}$ & Duration & Cause of death & Comment \\
\hline Denman et $a l^{45}$ & $\begin{array}{l}\text { Rheumatoid } \\
\text { arthritis } \\
\text { neuropathy }\end{array}$ & $\mathbf{F}$ & 66 & 100 & 12 weeks & $\begin{array}{l}\text { Bone marrow } \\
\text { suppression }\end{array}$ & Chlorambucil \\
\hline Lorenzen et $a l^{129}$ & $\begin{array}{l}\text { Rheumatoid } \\
\text { arthritis }\end{array}$ & - & - & - & Few weeks & Pancytopenia & Prednisone \\
\hline \multirow[t]{4}{*}{ Tausch $e t a l^{212}$} & Polyarthritis & - & - & 50 & 7 weeks & $\begin{array}{l}\text { Amyloid } \\
\text { nephritis }\end{array}$ & $\begin{array}{l}\text { Pre-existing } \\
\text { disease }\end{array}$ \\
\hline & Polyarthritis & - & - & 50 & 8 weeks & $\begin{array}{l}\text { Amyloid } \\
\text { nephritis }\end{array}$ & $\begin{array}{l}\text { Pre-existing } \\
\text { disease }\end{array}$ \\
\hline & Polyarthritis & - & - & 50 & 18 weeks & $\begin{array}{l}\text { Interstitial } \\
\text { pyelonephritis }\end{array}$ & $\begin{array}{l}\text { Pre-existing } \\
\text { disease }\end{array}$ \\
\hline & & & & & & Site of neoplasm & \\
\hline Cade $e t a l^{25}$ & $\begin{array}{l}\text { Rheumatoid } \\
\text { arthritis }\end{array}$ & $F$ & 44 & 75 & 4 years & $\begin{array}{l}\text { Carcinoma of } \\
\text { the cervix }\end{array}$ & \\
\hline
\end{tabular}


during treatment. ${ }^{45129212}$ These five patients, plus two who developed malignancy, are summarised in table 2 . The three patients reported by Tausch $e t a^{212}$ had renal dysfunction before immunosuppressive therapy, and it is doubtful that their deaths during treatment were caused by the drug.

The special concern about the induction of neoplasia in patients receiving this immunosuppressive thiopurine is the subject of other presentations in these proceedings. While we are aware of 18 individual case reports of malignancy in RA patients who have received azathioprine, the total number of patients treated is unknown. This review of 542 patients from 24 studies identified only two cases of carcinoma, an incidence of $0.4 \%$. This may be compared with estimates of 0.6 to $2.6 \%$ malignancy in all patients with RA.

\section{Discussion}

The total frequency of adverse reactions in patients with RA treated with azathioprine compares favour- ably to the incidence of toxicity with other slowacting, remission inducing agents. Physicians report unacceptable side-effects, or significant toxicities requiring withdrawal, in a significant percentage of patients and $1 \%$ of patients may develop lifethreatening toxicity.

The six studies published in 1976 to $1977^{1218255680177}$ show a lower incidence of leucopenia of $10 \%$, and new treatment programmes are designed to follow recommendations for using lower doses of azathioprine (1.0 to a maximum of 2.5 $\mathrm{mg} / \mathrm{kg} /$ day). Investigators have suggested temporary interruption of treatment, until white blood cell counts return to normal, and perhaps restarting the drug at half dosage. Dose modifications for controlling white counts, in patients taking 6-mercaptopurine or 6-thioguanine, are well known. However, in contrast to the treatment of malignant diseases, intentional suppression of peripheral leucocyte counts by progressive increase in dose is not considered to be the critical determinant of therapeutic response in RA. 


\section{Discussion}

DR J L DECKER (NIH-USA)

We have had a very edifying morning, hearing a very thorough review of the latest available information, which I think most of us are trying to apply practically to our patients. I solicit your questions, or your comments, on anything that you have heard today.

\section{DR BEGUIN (PARIS, FRANCE)}

I would like to ask, in view of the time during which these drugs have been used, and we now have five or six years' experience, which are nevertheless very dangerous, why are experiments continued in humans? We know that there is a certain percentage of deaths and a higher percentage of complications, which may be serious, and which leave the patient much more worried by these complications than by their polyarthritis. I wish to know how we may press for these experiments to be limited; they are also very expensive for the laboratories.

\section{DR DECKER}

As I understand it, the commentator categorises much of what you have heard this morning as experiments and asks if they should be continued. My response would be that rheumatoid arthritis is a terrible disease and patients need something. Accordingly, I suppose that most of us in the room feel that the risks that have been described today, the expense, the laboratory studies, etc, are justified in an attempt to make their lives more bearable. You have to die somehow ...

\section{DR B CORRIGAN (SYDNEY, AUSTRALIA)}

Could we hear something of the effects of time on treatment. In other words, is there an optimal treatment time, or time after which azathioprine should not be used at all? Are there any data on the effects of time on the side-effects? We had one particular instance of malignancies tending to occur early. It strikes me that everybody is looking now for tumours occurring later. What is the effect of time and dosage on the problem of malignant side-effects?

\section{DR DECKER}

The time to begin treatment would presumably be a function of the disease. I suppose that there are some people who have got into enough trouble with gold, or something else, within the first year of their rheumatoid arthritis, so that it might be appropriate to begin drugs of this category then. When should we begin to look for malignancies? We have heard $\mathrm{Dr}$ Urowitz suggest that these neoplasms were already in the incipient phase, in three females with genital neoplasms, when the drug was begun. As they were followed, one might say that the incidence of malignancy was dropping. Do I interpret your remark correctly.

\section{DR M B UROWITZ (TORONTO, CANADA)}

That might be reading more into the data than is there, but that is what the data show. The malignancies that occurred, occurred early, and this is similar to the experience seen in the renal transplant situation, when the malignancies tend to occur within the first two years. I mentioned those two women who insisted on going back on azathioprine after they had their hysterectomies for treatment of carcinoma of the uterus and cervix. They have been followed now for almost a further two years and have no developed a recurrence or another malignancy. From that, I drew the inference that, maybe, they had a premalignant lesion there. I don't think that we can ever let up our vigilance. If, after $1 \frac{1}{2}$ or two years, there is no malignancy, you cannot stop looking. The purpose of these large, on-going, and often retrospective studies, is to see whether the incidence is at all increased and, if it is increased, is it increased early or late? I think only large studies will give us these answers.

\section{DR DECKER}

This to some extent depends on the drug involved as well. I think that with alkylating agents more extended concern is warranted, even after the drugs are stopped. I think Dr de Silva has made it very clear that the dose of chlorambucil over time is also very pertinent to the potential development of malignancy.

\section{DR H DEICHER (HANOVER, GERMANY)}

I have a question relating to the increased incidence of lymphoreticular tumours which has been referred to by some of the speakers here this morning. I mean the increased incidence of lymphoreticular tumours in diseases like lupus and others. Since we have $\mathrm{Dr}$ Denman with us, who has already provided us with a 
number of interesting assumptions, I should like to ask him whether he has an explanation of the increased incidence of this type of tumour in these so-called autoimmune diseases.

\section{DR DECKER}

Dr Denman, before you respond, Dr Barnes would like to add to your troubles.

\section{DR C G BARNES (LONDON, UK)}

I wonder if I might also ask Dr Denman what he thinks is the relevance of Sjögren's syndrome in association with rheumatoid arthritis in these patients. We know that some patients with Sjögren's syndrome have an increased propensity for lymphoreticular problems. I find it difficult that in many reports of such tumours in patients treated with various agents the incidence of Sjögren's syndrome is never stated.

\section{DR A M DENMAN (NORTHWICK PARK UK)}

I think that it is evident from what has been said that we are speculating about the nature of diseases such as rheumatoid arthritis. Thus, I can only pile speculation on speculation. But let us start with two clear observations. The first point is that the incidence of lymphoreticular tumours in patients who have received cytotoxic drugs following renal and other transplants is much higher than that observed in patients receiving the same drugs for the treatment of autoimmune and chronic rheumatic diseases. The transplants provoke persistent lymphoproliferative activity which, in combination with mutagenic drugs, leads to neoplasia. The second point is that even potent carcinogens in animals have a long latent period before tumours are evident, and even then only certain organs are affected. Thus if one takes a very potent carcinogen such as $\mathrm{O}^{6}$ methylnitrosourea (MNU), and gives it to strains of mice which are either sensitive or resistant to the oncogenic effects of this agent, there is a long latent period before tumours emerge and only certain sites are involved, as Phil Lawley has clearly shown. His studies have emphasised that two factors predispose, above all, to the development of cancer in these mice. The first is the extent of proliferative activity which goes on in the target cells for neoplastic transformation. Thus, the thymus has a high level of lymphoproliferative activity and is particularly susceptible to tumours induced by MNU. It is interesting to reflect that lymphoreticular tumours are more likely to occur in younger patients and in systemic lupus erythematosus (SLE) rather than rheumatoid arthritis, since in the former conditions there is a high rate of spontaneous lymphoproliferative activity. Thus, cultured B lymphocytes from patients with SLE produce large amounts of immunoglobulins spontaneously.

The second factor is the extent to which different tissues can repair the damage induced by MNU in the experimental system and by cytotoxic drugs such as alkylating agents in clinical practice. More attention has to be paid than in the past to such repair mechanisms. What is peculiar about the thymus, which is cancer-sensitive in mice receiving powerful mutagens, as opposed to other organs which appear to be resistant to tumour induction, is the poor ability of the thymus to remove promutagens from the DNA of thymus cells. The thymus in mice which are cancer prone is inefficient at removing alkylating agents, whereas other tissues like the liver are extremely efficient. What may save human patients from the oncogenic effects of cytotoxic drugs is the much greater efficiency of human lymphocytes, compared with murine lymphocytes, in removing promutagens. This point has been emphasised by the results of collaborative studies with Phil Lawley and Gilmour Harris of the Kennedy Institute. Lymphocytes from patients with autoimmune diseases may show impoverished ability to remove promutagens compared with lymphocytes from normal subjects, but this deficiency is not absolute. Lymphocytes bearing potentially malignant mutations may also be killed by continuous exposure to cytotoxic drugs. These are the sort of areas we should explore in the future, but for the moment what I have said remains mainly speculative.

\section{DR DECKER}

In connection with Dr Barnes's remarks, you would count Sjögren's syndrome as a disease of lymphoproliferation, in some degree, in its own right, and that makes patients more prone, perhaps.

\section{DR DENMAN}

One is always worried that an audience like this could contain people such as Keith Whaley or Norman Talal, who know far more about the subject than I do. However, it is most people's impression that the emergence of lymphomas in Sjögren's syndrome is the end stage of a lymphoproliferative process, which is initially polyclonal and ends up as a B cell lymphoma originating in a single malignant clone. If one believes that there is some association between the $B$ cell aberrations which produce autoantibodies and those which produce lymphoma, it is not unexpected that one process should evolve into the other in some patients. 


\section{Suppl p 50 Annals of the Rheumatic Diseases}

\section{DR W W BUCHANAN (HAMILTON, CANADA)}

I think one should inject just a word of caution about these drugs. They are described as 'slow-acting' drugs, but they are also, we should remember, weak-acting drugs. When one looks at the clinical trials that have been done, for instance of gold, which was Dr Ward's standard 'second-line' drug, one finds in fact that the results indicate it limping ahead of placebo. The Empire Rheumatism Council trial ${ }^{63}$ showed a difference between the means of the number of active joints in the gold treated people of nine, and in the control group of six, which is hardly a dramatic effect. Grip strength increased by $30 \mathrm{mmHg}$ in the gold treated patients and $10 \mathrm{mmHg}$ in the placebo treated patients. So I would first make the point, we are really dealing not only with a slowacting but a weak-acting drug.

The second point is the toxicity of gold which $\mathrm{Dr}$ Paulus reviewed so well: one death in 10000 prescriptions. We should recall that chloramphenicol does that at the rate of 1 in 30000 and it was removed from the pharmacopoeia in the UK as a result, and phenylbutazone in fact kills 1 in 80000 . So this, then, from that data, would be a very toxic drug indeed. One should recall that the data that he was basing that on was voluntary, not obligatory, recording of deaths so in fact we may be dealing with a much more toxic drug than we would like to believe.

\section{DR DECKER}

I thought Dr Paulus made the point very well that these drugs were, in fact, hardly perfect for rheumatoid arthritis. We are all aware of their failures, Dr Buchanan, and one of the reasons, I think, we try them is because there are small numbers of patients out there, on the far extreme away from the mean, that do magnificently and one is always hoping that each patient will do magnificently. Certainly the population is not uniform in behaviour. This is a very interesting issue which we have been running into in the lymphopheresis work. It is quite clear that what we describe as active rheumatoid arthritis is not the same thing in all patients. I mean, the immunological events going on in 15 patients with active RA-nodules, systemic involvement, synovitis-are different and we are going to have different effects of these medications. This is one of the reasons why it is so important to try to look for predictors, so that we can pick out people who will respond, and not subject the others to the risk of whatever form of treatment you are dealing with.

\section{DR S ALEXANDER (CALIFORNIA, USA)}

Pursuing the first question of Dr Corrigan, there was a report from Dr Isomaki's group from Heinola, Finland, ${ }^{132}$ indicating that the gold salts were much more effective if used in the first two years, that is when used early, and in fact when used after the disease process had been present for longer than two years were not effective at a statistically significant level. I wonder if any of the panelists have any information to bear on this, as to whether this would be confirmed; because if it is true, it would seem that it would invalidate the results of most of these studies and perhaps the effectiveness would not be as bleak as Dr Buchanan has pointed out.

\section{DR DECKER}

Would anybody care to respond to that?

\section{DR H A ISOMAKI (HEINOLA, FINLAND)}

I would like to comment. As to the effect of gold, we don't know if it depends on the effect of the drug itself or if it is that patients come frequently to the doctor who sees and takes good care of them. I think that at least part of the therapeutic effect of gold is the result of good care of patients. The doctor sees them very often and, of course, if you start treatment early, at the beginning of the disease, you must get better results than if you start it later. If you start aftexô erosions occur you cannot prevent them.

\section{DR M D SKEITH (SEATTLE, USA)}

A practical point, since we're always looking, Dr Decker, for the patient who responds very well to these medications. Is there anyone on the panel who would like to comment about the duration of time that one should persist in the use of any of these individual drugs-say azathioprine for instancebefore one abandons it as being an ineffective drug? Would that be three months, six months, or two years?

\section{DR DECKER}

I suppose we could have a good long discussion on just that point. Dr Paulus, would you care to respond?

\section{DR H E PAULUS (LOS ANGELES, USA)}

That is always a difficult decision to make when one runs along for three months, or six months, or a year with a patient on a particular drug and who still has active disease. One of the points that I was trying to make in my talk was that the sequential use of a number of slow-acting drugs is of little benefit. It may take a year before you see a major benefit from a drug. If you stop at that time or earlier and then you start another one, it takes another six months, or 
longer, before you can expect to see a response. In a totally non-statistical way, in patients that I follow, I frequently see patients on gold, say after a year of therapy, who continue to have symptomatically active disease and they are not very happy with how they are doing. Usually I try to carry on, but sometimes the patient convinces me to stop. So we stop and switch to something else, and the patient often gets a lot worse within the next three or six months, before he sometimes gets better with the second drug. So my tendency would be to try to carry on a lot longer than we usually do.

\section{DR WHISNANT (BURROUGHS WELLCOME, USA)}

In the US prescribing advice, the data allow you to treat for 12 weeks and, if there is no response by that time, current studies say that you are at the limit of the data. Let me just remind you that Dr Ward's co-operative study was designed to try to extend that to a six-month treatment period, which we think has probably got to be a minimum time to try to assess an individual patient's response.

\section{DR BARNES}

I remind you that one follow-up study in the United Kingdom showed progressive improvement up to sometimes as long as one year.

\section{DR M DE SILVA (CAMBRIDGE, UK)}

We have evidence of the continued effect of azathioprine after five years of continuous use. We have withdrawn azathioprine and we have shown that even after five to eight years of continuous use, in a double blind manner, if you discontinue the drug the disease reactivates and it responds to the reintroduction of the drug. So the answers to the first and subsequent questions are that azathioprine continues to exert a beneficial effect over a prolonged period-and our study also included combination with gold-and after withdrawal and reintroduction it can again be effective.

\section{DR UROWITZ}

I think that there is a little confusion here with the question. One question is, what do you do with the patient who does not respond? I think Dr Whisnant's comments are right, that the data would indicate that by 12 weeks there is definitely some response if there is going to be a response. If there is not any clinical response by 12 weeks, it is probably not worth pursuing azathioprine. On the other hand, if a patient does get a response, how long should you continue that drug? Of the patients who have already begun to respond, all the studies have now shown that as you follow them further and further down the line they maintain and even improve on that response. So there are two answers: if they respond, continue; if they have not responded by 12 weeks, you are probably at the end of the trial.

\section{DR GONZALEZ (CANARY ISLANDS, SPAIN)}

When a patient with rheumatoid arthritis does well with gold, do you stop or do you maintain the gold therapy? How long do you maintain the gold therapy?

\section{DR DECKER}

The question is, with a good response to gold therapy, how long does one continue it? I suppose that everyone in this room would have a different response to that question. For myself, when I have got a solid remission, I have continued it for life at the rate of $100 \mathrm{mg}$ sodium aurothiomalate (Myocrisin) every three months. It may be a placebo, but I would continue gold until I got a complete relapse that demanded a change to go to something else or I got into toxicity-which, as a rule, you don't get after years like that, although you can. You did not examine any $x$-rays in that AB study, Dr Ward?

\section{DR WARD (SALT LAKE CITY, USA)}

No!

\section{DR A J GOLDBERG (LONDON, UK)}

I wonder if I could ask a question of the panel about second courses of treatment with the same drug. It is our experience that a second course of gold is very often not as successful as the first. The question would first of all be, why does the panel think that might be so, and secondly what is the panel's experience with second courses of other disease modifying agents as far as success is concerned?

\section{DR UROWITZ}

In terms of the cytotoxics, I think that I've probably answered, as if I haven't had a response after 12 weeks, I give up a cytotoxic.

\section{DR GOLDBERG}

But what if a patient had been doing well and wanted to go back on it?

\section{DR UROWITZ}

Oh yes, I would have no hesitation in restarting. And then your question is the specific side-effects it was stopped for? 


\section{DR GOLDBERG}

No, my question is, what is the likelihood of a successful response to a second course of therapy if the first one was successful and was stopped for side-effects, but the patient, for example, wanted to start again. We know that with gold, the second course is often not very successful. What is the experience with other cytotoxics-other disease modifiers?

\section{DR UROWITZ}

For instance, with azathioprine, patients who have been stopped for leucopenia and have flared, or patients who have stopped because we were afraid to carry them on beyond one or two years in our early studies, and then flared, have been restarted. They have responded and we have actually reported that. So that the patients who stopped, because of our fear, have been recaptured.

\section{DR PAULUS}

I would agree. I think that a fair number of patients with gold will have a second response if they are restarted carefully following the resolution of a toxicity.

\section{DR DECKER}

I think that we are reaching the end of our comments. Dr Hitchings.

\section{DR G H HITCHINGS (BW \& CO, USA)}

Much of what we have heard today, including empirical studies of a clinical nature, shows that even after two or three decades we have not come to definitive treatments. We are beginning to see glimmers by sorting out cell types-cell types that are responsive to specific drugs. We are beginning to find glimmers of what kind of biochemistry goes on in these responding cells. I would like to predict that the next decade is going to see some revolutionary changes in this field.

\section{DR DECKER}

I am sure they will be welcome. 


\title{
Conclusions
}

\author{
COLIN G BARNES AND JOHN L DECKER
}

The problem of adequately suppressing disease activity in rheumatoid arthritis remains with us, and it is acknowledged that currently available drugs are only effective in a proportion of patients. These drugs do not eradicate the disease, their effect in some patients may only be modest and all are potentially toxic.

The immunological basis for the use of the immunosuppressive agents is well known and the rationale for their use has been reviewed in these proceedings by Dr Hitchings and Dr Denman, both of whom envisage substantial developments in this form of treatment in the future.

Again, the clinical efficacy of gold and azathioprine has been reviewed, indicating long-term effect of these drugs in suppressing rheumatoid inflammatory activity. The point has been stressed, however, that to hope to obtain lasting benefit for the patient, suppressive medication must be continued for many months or years. Increasing benefit to the patient may continue for one or more years after the drug has been started, and thus, in a chronic progressive disease, short courses of drugs which may modify the disease process are unlikely to prove effective in modifying the long-term progress of the disease. It is difficult to achieve suitable radiological comparison groups over extended time periods, but azathioprine over 18 months has been associated with less $x$-ray deterioration than that observed with gold. ${ }^{38}$

In view of the potential toxicity of this form of treatment, and the need to continue treatment longterm, much of the discussion has concentrated on toxic effects. Of these, gastrointestinal intolerance to oral medications and bone marrow suppression, the latter virtually always reversed by dose reduction, are well known. These have again been reported in a substantial proportion of cases. However, the possible potential for the 'immunosuppressive agents' to induce neoplasia has been reviewed in as much detail as is presently available. The results remain inconclusive with sporadic reports of commonly occurring solid tumours developing in patients while receiving azathioprine or other antimitotic agents. Uncertainties as to the incidence of cancer in rheumatoid patients who have not received cytotoxic drugs are sufficiently great, and the incidence in azathioprine treated rheumatoids is so low, as to necessitate further controlled analysis of the problem. There has not been any substantial incidence of lymphoreticular tumours reported as is known to occur in patients receiving these drugs after renal transplantation.

Thus, this workshop has served to review the current knowledge on the effect and toxicity of drugs aimed at suppressing rheumatoid disease, with the conclusion that all of these drugs - gold salts, penicillamine and immunosuppressives (azathioprine and cyclophosphamide in particular)-may be partially effective but toxic. Although the immunosuppressives still require further evaluation regarding oncogenesis and.studies are continuing, there do not seem to be any notable differences in frequency or severity of toxic reactions when one drug is compared with another. The alkylating agents may present a slightly increased risk. Until more effective and less toxic drugs are developed to suppress rheumatoid disease in our patients, we continue to have to use available agents to improve their clinical situation, realising the need for careful supervision during long-term treatment. 


\section{References}

${ }^{1}$ Abel T, Urowitz MB, Smythe HA, Keystone EC, Norman CS. Longterm effects of azathioprine in rheumatoid arthritis. Arthritis Rheum 1978; 21: 539.

${ }^{2}$ Alexson E, Brandt KD. Acute leukemia after azathioprine treatment of connective tissue disease. Am J Med Sci 1977; 273: 335-40.

${ }^{3}$ Allison AC, Hove T, Watts RWE, Webster ADB. Immunological observations on patients with LeschNyhan syndrome, and on the role of de novo purine synthesis in lymphocyte transformation. Lancet 1975; ii: $1178-82$

4 Alpert ME, Uriel J, deNechaud B. Alpha-1fetoglobulin in the diagnosis of human hepatoma. $N$ Engl J Med 1968; 278: 984.

5 Amor B, Mery C. Chlorambucil in rheumatoid arthritis. Clin Rheum Dis 1980; 6: 567-84.

${ }^{6}$ Anderson LG, Talal N. The spectrum of benign to malignant lymphoproliferation in Sjögren's syndrome. Clin Exp Immunol 1972; 10: 199-221.

7 Apostoloff E, Reitzig P, Jendrusch C, Apostoloff G. Experience with long-term immunosuppressive treatment of diseases of the connective tissue. $Z$ Gesamte Inn Med 1974; 29: 26-30 (translation).

${ }^{8}$ Aptekar RG, Steinberg AD, Decker JL. Complications of cytotoxic agents in systemic lupus erythematosus and rheumatoid arthritis. Arthritis Rheum 1973; 16: 533 (abst).

9 Armitage P. Statistical methods in medical research. Oxford: Blackwell Scientific Publications, 1971: 388-91.

10 Asherson GL, Webster ADB. Clinical immunodeficiency diseases. Oxford: Blackwells, 1980.

${ }^{11}$ Badley EM, Ball J, Wood PHN. Interpreting the biological significance of rheumatoid factor. Ann Rheum Dis 1975; 34: 463.

12 Balken WJ. Therapy of progressive chronic polyarthritis. Clinical studies of the effectiveness of azathioprine. Fortschr Med 1976; 94: 1504-6 (translation).

${ }^{13}$ Ball J. Postmortem findings and articular pathology in rheumatoid arthritis. In: Duthie JJR, Alexander WRM, eds. Rheumatic diseases. Pfizer Medical Monograph No 3. Edinburgh: Edinburgh University Press, 1968: 124-6.

${ }^{14}$ Barnes CG. Immunosuppressive therapy in rheumatoid arthritis. In: Miescher PA, ed. Immunosuppressive therapy. Proceedings of the Wiesbaden Symposium 1972. Basel, Stuttgart: Schwabe, 1980: 57-68.

15 Barnikol HU, Vorlaender KO. Prerequisites and initial results with the therapeutic administration of azathioprine (Imuran) in chronic-progressive articular rheumatism and the so-called collagenoses. Med Welt 1967; 3: 160-9 (translation).

${ }^{16}$ Batchelor JR, Welsh KI, Tinoco RM, et al. Hydrallazine induced systemic lupus erythematosus: the influence of HLA-DR and sex upon susceptibility. Lancet 1980; 1: 1107-9.

${ }^{17}$ Bennett JC. The infectious aetiology of rheumatoid arthritis. Arthritis Rheum 1978; 21: 531-8.

${ }^{18}$ Berry H, Liyanage SP, Durance RA, Barnes CG,
Berger LA, Evans S. Azathioprine and penicillamine in treatment of rheumatoid arthritis: a controlled trial. $\mathrm{Br}$ Med J 1976; i: 1052-4.

${ }^{19}$ Box GEP, Hunter WG, Hunter JS. Statistics for experimenters. New York: Wiley Scientific Publications, 1978: 144-5.

${ }^{20}$ Boyle DJ, Day JF, Kassan SS, Thomas MR, Robinson WA, Steigerwald JC. Incidence of malignancy 10 years following cyclophosphamide use for rheumatoid arthritis. Paper presented before the American Rheumatism Association, Boston, Massachusetts, June 3, 1981.

${ }^{21}$ Brinkley JR Jr, Dubois EL, Ryan SJ. Long-term course of chloroquine retinopathy after cessation of medication. Am J Ophthalmol 1979; 88: 1-11.

${ }^{22}$ Bruckner I, Stroescu O, Stroescu V, et al. Investigations on the effect of azathioprine (Imuran) in rheumatoid arthritis and lupus erythematosus disseminatus. Rev Roum Med Intern 1969; 1: 19-30.

${ }^{23}$ Buriot D, Prieur AM, Lebranchu Y, Messerschmitt J, Griscelli C. Leucémie aigue chez trois enfants atteints d'arthrite chronique juvenile traités par chlorambucil. Arch Fr Pediatr 1979; 36: 592-8.

${ }^{24}$ Bywaters E, Curwen M, Dresner E, Dixon AStJ. 10 year follow up study of rheumatoid arthritis Ann Rheum Dis 1961; 20: 198.

${ }^{25}$ Cade R, Stein G, Pickering M, Schlein E, Spooner G. Low-dose, long-term treatment of rheumatoid arthritis with azathioprine. South Med J 1976; 69: 388-92.

${ }^{26}$ Calabro JJ. Cancer and arthritis. Arthritis Rheum 196 10: 553-67.

${ }^{27}$ Caldwell DS. Musculoskeletal syndromes associate with malignancy. Semin Arthritis Rheum 1981; 10: 198.

${ }^{28}$ Cammarata RJ, Rodnan GP, Jenson WN. Systemic rheumatic disease and malignant lymphoma. Arch Intern Med 1963; 3: 330-7.

${ }^{29}$ Canoso JJ, Cohen AS. Malignancy in a series of 70 patients with systemic lupus erythematosus. Arthritis Rheum 1974; 17: 383-90.

${ }^{30}$ Chang J, Geary CG. Therapy-linked leukaemia. Lancet 1977; i: 97.

${ }^{31}$ Chanmougan D, Schwartz RS. Enhancement of antibody synthesis by 6-mercaptopurine. J Exp Med 1966; 124: 363-78.

${ }^{32}$ Cobau CD, Sheon RP, Kirsner AB. Immunosuppressive drugs and acute leukemia. Ann Intern Med 1973; 79: 131-2.

${ }^{33}$ Cobb S, Anderson F, Bauer W. Length of life and cause of death in rheumatoid arthritis. N Engl J Med 1953; 249: 553-6.

${ }^{34}$ Cooperating Clinics Committee of the American Rheumatism Association. A controlled trial of cyclophosphamide in rheumatoid arthritis. $N$ Engl J Med 1970; 283: 883-9.

${ }^{35}$ Cooperating Clinics Committee of the American Rheumatism Association. A controlled trial of high and low dose of cyclophosphamide in 82 patients with rheumatoid arthritis. Arthritis Rheum 1972; 15: 434.

${ }^{36}$ Cooperman JM. Microbiological assay of serum and wholeblood folic acid activity. Am J Clin Nutr 1967; 20: 1015.

${ }^{37}$ Court-Brown WM, Doll R. Leukaemia and aplastic anaemia in patients irradiated for ankylosing spon- 
dylitis. MRC Special Report Series No 295. London: HMSO, 1957.

${ }^{38}$ Currey HLF, Harris J, Mason RM, et al. Comparison of azathioprine, cyclophosphamide and gold in treatment of rheumatoid arthritis. $\mathrm{Br}$ Med J 1974; iii: 763-6.

${ }^{39}$ Dale GA, Smith RB. Transitional cell carcinoma of the bladder associated with cyclophosphamide. J Urol 1974; 112: 603-4.

${ }^{40}$ Davis P. Undesirable effects of gold salts. J Rheumatol 1979; 6 (suppl 5): 18-24.

${ }^{41}$ DeBoer KP, Kleinman R, Teodorescu M. Identification and separation by bacterial adherence of human lymphocytes that suppress natural cytotoxicity. $J$ Immunol 1981; 126: 276-81.

42 Decker JL. Chairman's summary of session V. Evaluation of DMARD's. In: Paulus HE, Ehrlich GM, eds. Symposium Medica Hoechst. Controversies in the clinical evaluation of analgesic/anti-inflammatory/antirheumatic drugs. Stuttgart, New York: Schattaur Verlag, 1981: 378-9.

${ }^{43}$ Denman AM. Viruses and autoimmune diseases. In: Holborow EJ, ed. Autoimmunity. Clinics in Immunology and Allergy 1. London: Saunders, 1981: 17-39.

${ }^{44}$ Denman AM, Hollingworth P, Tyndall AdeV, et al. Intensive immunosuppression versus prednisone in the treatment of connective diseases. Ann Rheum Dis (in press).

${ }^{45}$ Denman EJ, Denman AM, Greenwood BM, Gall D, Heath RB. Failure of cytotoxic drugs to suppress immune responses of patients with rheumatoid arthritis. Ann Rheum Dis 1970; 29: 220-31.

${ }^{46}$ de Seze S, Kahn M. L'association de produits à l'action cytolitique aux corticostéroides dans le traitement des rhumatismes inflammatoires chroniques. In: Rhumatismes inflammatoires chroniques. Lym Trevoux, 1965.

${ }^{47}$ Deshayes P, Renier JC, Bregeon C, Hondent G. Incidents et accidents des thérapeutiques à visée immunodepressive dans la polyarthrite rhumatoide. Rev Rhum Mal Osteoartic 1971; 38: 797.

48 Dixon AStJ, Davies J, Dormandy TL, et al. Synthetic $\mathrm{d}(-)$ penicillamine in rheumatoid arthritis: doubleblind controlled study of a high and low dosage regimen. Ann Rheum Dis 1975; 34: 416-21.

49 Dixon AStJ, Lindsay DJ, Collins EM. Immunosuppressive drugs in rheumatoid arthritis. (Letter.) $\mathrm{Br} \mathrm{Med} \mathrm{J}$ 1971 ; i: 460 .

${ }^{50}$ Dixon FJ, Theofilopoulos AN, Izul S, McConahey PJ. Murine SLE-aetiology and pathogenesis. In: Fougereau M, Dausset J, eds. Immunology 80. Progress in Immunology IV. London: Academic Press, 1980: 959-95.

${ }^{51}$ Dodson WH, Bennett JC. Possible usefulness of azathioprine (Imuran) in severe rheumatoid arthritis. $J$ Clin Pharmacol 1969; 9: 251-8.

52 Dubois EL. Lupus erythematosus: a review of the current states of discoid and systemic lupus erythematosus and their variants. New York: McGraw-Hill, 1966.

${ }^{53}$ Duclos H, Maillot MC, Kreis H, Fries D, Galanaud P. $T$ suppressor cell function impairment in peripheral blood lymphocytes from transplant recipients under azathioprine and corticosteroids. Transplantation 1979; 28: 437-8.

${ }^{54}$ Duclos H, Maillot MC, Kreis H, Fries D, Galanaud P. Depressed $\mathrm{T}$ suppressor cell function in PBL of transplanted patients under azathioprine and corticosteroids. Transplant Proc 1979; 11: 1246.

${ }^{55}$ Duthie JJR, Brown PE, Truelove LH, Naragar FD, Lawrie AJ. Course and prognosis in rheumatoid arthritis. A further report. Ann Rheum Dis 1964; 23: 193.

${ }^{56}$ Dwosh IL, Stein HB, Hunter T, Urowitz MB, Smythe HA, Ogryzlo MA. Azathioprine in early rheumatoid arthritis: a comparison study with gold and chloroquine. Arthritis Rheum 1977; 20: 685-92.

${ }^{57}$ Ederer F, Mantel N. Confidence limits on the ratio of two Poisson variables. Am J Epidemiol 1974; 100: 165-7.

${ }^{58}$ Editorial. Renal transplantation in the 1980s. Br Med J 1980; 280: 503-4.

${ }^{59}$ Elion GB, Callahan SW, Bieber S, Hitchings GH, Rundles RW. A summary of investigations with 6[(1-methyl-4 nitro-5-imidazolyl)] thiopurine. Cancer Chemother Rep 1961; 14: 93-8.

${ }^{60}$ Elion GB, Hitchings GH. Azathioprine. In: Sartorelli AC, Johns DG, eds. Antineoplastic and immunosuppressive agents II. Berlin, Heidelberg, New York: Springer Verlag, 1975: 404-25.

${ }^{61}$ Elliot GB, Silverberg DS, Dossetor JB, Muir CS. Latent carcinoma of the prostate in a 24 year old man receiving cyclophosphamide and azathioprine. Can Med Assoc J 1977; 116: 651-2.

${ }^{62}$ Empire Rheumatism Council. Gold therapy in rheumatoid arthritis: report of a multicentre controlled trial. Ann Rheum Dis 1960; 19: 95-119.

${ }^{63}$ Empire Rheumatism Council. Gold therapy in rheumatoid arthritis: final report of a multicentre controlled trial. Ann Rheum Dis 1961; 20: 315-34.

${ }^{64}$ Farber SJ, Sheon RP, Kirsner AB, Finkel RI. Incidence of malignant diseases in patients receiving cytotoxic therapy for rheumatoid arthritis. Arthritis Rheum 1979; 22: 608.

${ }^{65}$ Farrant J, Knight SC. Help and suppression by lymphoid cells as a function of cellular concentration. Proc Natl Acad Sci USA 1979; 76: 3509-13.

${ }^{66}$ Feng PH, Cheach PS, Lee YK. Mortality in systemic lupus erythematosus: a ten year review. Br Med J 1973; iv: $772-4$.

${ }^{67}$ Fialkow PJ, Denman AM, Singer J, Jacobson RJ, Lowenthal MN. Human myeloproliferative disorders: clonal origin in pluripotent stem cells. In: Differentiation of normal and neoplastic haematopoietic cells. Cold Spring Harbor Laboratory 1978: 131-44.

${ }^{68}$ Forni AM, Koss LG, Geller W. Cytological study of the effect of cyclophosphamide on the epithelium of the urinary bladder in man. Cancer 1964; 17: 1348-55.

${ }^{69}$ Fosdick WM, Parsons JL, Hill DF. Longterm cyclophosphamide therapy in rheumatoid arthritis. Arthritis Rheum 1968; 11: 151-61.

${ }^{70}$ Fraser TN. Gold therapy in rheumatoid arthritis. Ann Rheum Dis 1945; 4: 71-5.

${ }^{71}$ Freedman A, Steinberg VL. Chloroquine in rheumatoid arthritis: a double-blindfold trial of treatment for 1 year. Ann Rheum Dis 1960; 19: 243-50. 
${ }^{72}$ Fricke R, Deicher H. Studies on therapy of rheumatoid arthritis (progressive chronic polyarthritis) with cytostatic drugs. $Z$ Rheumaforschung 1969; 28: 20-9.

${ }^{73}$ Frisch AW, Davies GH. Inhibition of hemagglutinin formation by thioguanine: dose-time relationships. Proc Soc Exp Biol Med 1962; 110: 444-9.

${ }^{74}$ Fusco F, Mattioli F, Bertocchi I. Development of malignancy after immunosuppressive treatment (azathioprine). First Meeting of the European Division of the International Society of Hematology, Milan, September 1971.

${ }^{75}$ Gardner DL. Causes of death. In: The pathology of rheumatoid arthritis. London: Arnold, 1972: 183-7.

${ }^{76}$ Ghaffar A, Lichter W, Wellham LL, Paul RD, Sigel MM. Effects of various alkylating agents on T-dependent and T-independent immune responses. Proceedings of the 11th International Congress on Chemotherapy 1980; 2: 1716-7.

${ }^{77}$ Giblett ER, Ammann AJ, Wata DW, Sandman R, Diamond LK. Nucleoside-phosphorylase deficiency in a child with severely defective T-cell immunity and normal B-cell immunity. Lancet 1975; i: 1010-3.

${ }^{78}$ Giblett ER, Anderson JE, Cohen F, Pollara B, Meuwissen HJ. Adenosine-deaminase deficiency in two patients with severely impaired cellular immunity. Lancet 1972; ii: 1067-9.

${ }^{79}$ Gilmore IT, Holden GT, Rodan KS. Acute leukemia during azathioprine therapy. Postgrad Med J 1977; 53: 173-4.

${ }^{80}$ Goebel KM, Janzen R, Joseph K, Borngen U. Disparity between clinical and immune responses in a controlled trial of azathioprine in rheumatoid arthritis. Eur J Clin Pharmacol 1976; 9: 405-10.

${ }^{81}$ Goldenberg GJ, Paraskevas F, Israels LG. The association of rheumatoid arthritis with plasma cell and lymphocytic neoplasms. Arthritis Rheum 1969; 12: 569-78.

${ }^{82}$ Gordon DA, Smythe HA, Pruzanski MA, Ogryzlo MA. Azathioprine in rheumatoid arthritis. A doubleblind cross over study. Arthritis Rheum 1973; 16: 411-8.

${ }^{83}$ Grooten J, DeBaetselier P, Vercauteren E, Hamers R. Anti-micrococcus antibodies recognise an antigenic marker of confluent mouse lymphoid cell lines. Nature 1980; 285: 401-3.

${ }^{84}$ Gumpel JM, Hall A, Ansell B. A double-blind comparative trial of cyclophosphamide and gold in rheumatoid arthritis. Ann Rheum Dis 1974; 33: 574.

${ }^{85}$ Gupta PK, Pinn VM, Taft PD. Cervical dysplasia associated with azathioprine (Imuran) therapy. Acta Cytol (Baltimore) 1969; 13: 373.

${ }^{86}$ Gupta S, Good RA. Markers of human lymphocyte subpopulations in primary immunodeficiency and lymphoproliferative disorders. Semin Hematol 1980; 17: 1-29.

${ }^{87}$ Hamilton EBD, Scott JT. Hydroxychloroquine sulfate ('Plaquenil') in treatment of rheumatoid arthritis. Arthritis Rheum 1962; 5: 502-12.

${ }^{88}$ Harris G, Lawley PD, Hylton W, Denman AM, Asbery LJ. Blood mononuclear cells in auto-immune diseases are deficient in removal of $\mathrm{O}^{6}$-methylguanine from DNA. In preparation.
${ }^{89}$ Harris J, Jessop JD, Chaput de Saintonge DM. Further experience with azathioprine in rheumatoid arthritis Br Med J 1971; 4: 463-4.

${ }^{90}$ Hicks ND. An unusual form of leukemia associated with immunosuppressive therapy. Pathology 1973; 5: 77.

${ }^{91}$ Hitchings GH. Summary and concluding remarks. Fed Proc 1967; 26: 958-60.

${ }^{92}$ Hitchings GH, Elion GB. The role of antimetabolites in immunosuppression and transplantation. Accounts Chem Res 1969; 2: 202-9.

${ }^{93}$ Hoh KF, Dwosh IL, Ford PM, Anastassiades TP, Kelly HG. Drug-related disease remissions in rheumatoid arthritis. Arthritis Rheum 1981; 24: suppl 72.

${ }^{94}$ Hoover R, Fraumeni JF Jr. Risk of cancer in renal transplant recipients. Lancet 1973; ii: 55.

95 Hunter T, Urowitz MB, Gordon DA, Smythe HA, Ogryzlo MA. Azathioprine in rheumatoid arthritis: a long term follow up study. Arthritis Rheum 1975; 18: 15-20.

${ }^{96}$ Husain Z, Runge LA. Treatment complications of rheumatoid arthritis with gold, hydroxychloroquine, d-penicillamine, and levamisole. J Rheumatol 1980; 7: 825-30.

${ }^{97}$ Huskisson EC, Gibson TJ, Balme HW, et al. Trial comparing d-penicillamine and gold in rheumatoid arthritis. Ann Rheum Dis 1974; 33: 532-5.

${ }^{98}$ International symposium on levamisole in rheumatoic arthritis. J Rheumatol 1978; 5, suppl 4: 1-153.

${ }^{99}$ International symposium on penicillamine. Rheumatol 1981; 8, suppl 7: 1-181.

${ }^{100}$ International workshop and symposium on gold salts in the rheumatic diseases. J Rheumatol 1979; 6, suppl 5: 1-164.

${ }^{101}$ Isomaki HA, Hakulinen T, Joutsenlahti U. Excess risks of lymphomas, leukemia and myeloma in patients with rheumatoid arthritis. J Chron Dis 1978; 31: 691-6.

${ }^{102}$ Isomaki HA, Mutru O, Koota K. Death rate and causes of death in patients with rheumatoid arthritis. Scand J Rheumatol 1975; 4: 205-8.

${ }^{103} \mathrm{Jaffe}$ IA. The effect of penicillamine on the laboratory parameters in rheumatoid arthritis. Arthritis Rheum 1965; 8: 1064-79.

${ }^{104}$ Jensen MK. Chromosome studies in patients treated with azathioprine and amethopterin. Acta Med Scand 1967; 182: 445.

105 Jensen MK. Effect of azathioprine on the chromosome complement of human bone marrow cells. Int J Cancer 1970; 5: 147-51.

106 Jimenez-Diaz C, Lopez Garcia E, Merchante A, Perianes J. Treatment of rheumatoid arthritis with nitrogen mustard. Preliminary report. JAMA 1951; 147: 1418-9.

${ }^{107}$ Kahn MF, Arlet J, Bloch-Michel H, Caroit M, Chaouat Y, Renier JC. Leucémies aiguës après traitement par agents cytotoxiques en rhumatologie: 19 observations chez 2006 patients. Nouv Presse Med 1979; 8/17: 1393-7.

${ }^{108}$ Kahn MF, Arlet J, Bloch-Michel H, Caroit M, Chaouat $Y$, Renier JC. Le risque de leucose aiguë après traitement des rheumatismes inflammatoires chroniques et 
des connectivitiés par les cytotoxiques à visée immunosuppressive. Rev Rhum 1979; 46: 163-7.

${ }^{109}$ Katona G, Larazza O, Isai M. Objective evaluation of the anti-inflammatory effect of azathioprine in rheumatoid arthritis. Am J Rheum and Infl 1978; 1: 232-6.

${ }^{110} \mathrm{Katz}$ DH. Lymphocyte differentiation, recognition and regulation. New York, San Francisco, London: Academic Press, 1977.

${ }^{111}$ Kay AGL. Myelotoxicity of d-penicillamine. Ann Rheum Dis 1979; 38: 232-6.

112 Kean MF, Dwosh IL, Anastassiades TP, Ford PM, Kelly HG. The toxicity pattern of d-penicillamine therapy. Arthritis Rheum 1980; 23: 158-64.

${ }^{113}$ Keitel W. Ergebnisse und Erfahrungen zum immunosuppressiver Therapie der Rheumatoide Arthritis. Allerg Immunol (Leipz) 1976; 12: 303-5.

${ }^{114}$ Khanna V, Woodbury JFL. Usefulness of azathioprine in rheumatoid arthritis. Ann R Coll Phys Surg Canada 1973; 6: 60 (abst).

115 Kinlen LJ, Sheil AGR, Peto J, Doll R. Collaborative United Kingdom-Australasian study of cancer in patients treated with immunosuppressive drugs. $\mathrm{Br}$ Med J 1979; ii: 1461-6.

${ }^{116}$ Kinsella PL, Ebringer RW, Corbett M. Levamisole in RA-a double blind study. J Rheumatol 1980; 7: 288-92.

${ }^{117}$ Klein J, Juretic A, Baxevanis CN, Nagy ZA. The traditional and a new version of the mouse $\mathrm{H}-\mathrm{Z}$ complex. Nature 1981; 291: 455-60.

${ }^{118}$ Klein MB, Pereira FA, Kantor I. Kaposi sarcoma complicating systemic lupus erythematosus treated with immunosuppression. Arch Dermatol 1974; 110: 602-4.

${ }^{119}$ Kobayakawa T, Louis J, Izui S, Lambert PH. Autoimmune response to DNA, red blood cells and thymocyte antigens in association with polyclonal antibody synthesis during experimental African trypanosomiasis. $J$ Immunol 1979; 122: 296-330.

${ }^{120}$ Larson EL. Cyclosporin $A$ and dexamethasone suppress $\mathrm{T}$ cell responses by selectively acting at distinct sites of the triggering process. J Immunol 1980; 124: 2828.

${ }^{121}$ Lawler SD, Klouda PT, Hardisty RM, Till M. The HLA system in lymphoblastic leukaemia. $\mathrm{Br} \mathrm{J} \mathrm{Haematol}$ 1971; 21: 595-605.

${ }^{122}$ Lawley PD. DNA as a target of alkylating carcinogens. Br Med Bull 1980; 36: 19-24.

${ }^{123}$ Levy J, Paulus HE, Bangert R. Comparison of azathioprine (AZ) and cyclophosphamide (CPA) in the treatment of rheumatoid arthritis (RA). Arthritis Rheum 1975; 18: 412-3 (abst).

${ }^{124}$ Levy J, Paulus HE, Barnett EV, Sokoloff M, Bangert R, Pearson CM. A double-blind evaluation of azathioprine treatment in rheumatoid arthritis and psoriatic arthritis. Arthritis Rheum 1972; 15: 116-7.

${ }^{125}$ Lewis P, Hazleman BL, Hanka R, Roberts S. Cause of death in patients with rheumatoid arthritis with particular reference to azathioprine. Ann Rheum Dis 1980; 39: 457-61.

${ }^{126}$ Lewis RB, Castor CW, Kinsley RE, Bole G. Frequency of neoplasia in systemic lupus erythematosus and rheumatoid arthritis. Arthritis Rheum 1976; 19: 1256-60.

${ }^{127}$ Lipsemeyer EA. Development of malignant cerebral lymphoma in a patient with systemic lupus erythematosus treated with immunosuppression. Arthritis Rheum 1972; 15: 183-6.

${ }^{128}$ Lopez DM, Paul R, Herbert L, Wellham L, Lichter W. Selective effects of cyclophosphamide on different parameters of the immune response. Proceedings of the 11th International Congress on Chemotherapy 1980; 2: 1726-7.

129 Lorenzen I, Brun C, Videbaek A. Treatment of immunologic diseases with cytostatics. Acta Med Scand 1969; 185: 501-6.

${ }^{130}$ Louie S, Schwartz RS. Immunodeficiency in the pathogenesis of lymphoma and leukaemia. Semin Hematol 1978; 15: 117.

${ }^{131}$ Love RR, Sowa JM. Myelomonocytic leukaemia following cyclophosphamide therapy of rheumatoid disease. Ann Rheum Dis 1975; 34: 534-5.

${ }^{132}$ Luukkainen R. Chrysotherapy in rheumatoid arthritis: with particular emphasis on the effect of chrysotherapy on radiographical changes and on the optimal time of initiation of therapy. Scand J Rheumatol 1980; suppl 34: 1-56.

${ }^{133}$ Lyle WH. Penicillamine. Clin Rheum Dis 1979; 5: 569-601.

${ }^{134}$ McAdam L, Paulus HE, Peter JE. Adenocarcinoma of the lung during azathioprine treatment. Arthritis Rheum 1974; 17: 92-4.

${ }^{135}$ McConkey B, Amos RS, Durham S, Forster PJG, Hubball S, Walsh L. Sulphasalazine in rheumatoid arthritis. Br Med J 1980; i: 442-4.

${ }^{136}$ McConkey B, Davies P, Crockson RA, Crockson AP, Butler M, Constable TJ. Dapsone in rheumatoid arthritis. Rheumatol Rehabil 1976; 15: 230-4.

${ }^{137}$ McConkey B, Davies P, Crockson RA, Crockson AP, Butler M, Constable TJ, Amos RS. Effect of gold, dapsone and prednisone on serum C-reactive protein and haptoglobin and the erythrocyte sedimentation rate in rheumatoid arthritis. Ann Rheum Dis 1979; 38: $141-4$.

${ }^{138} \mathrm{McCracken}$ JD. Additional risk from immunosuppressive therapy for rheumatoid arthritis. Ann Intern Med 1973; 79: 611-2.

${ }^{139}$ Mackenzie AH, Scherbel AL. Chloroquine and hydroxychloroquine in rheumatological therapy. Clin Rheum Dis 1980; 6: 545-66.

${ }^{140}$ Mainland D, Sutcliffe MI. Hydroxychloroquine sulfate in rheumatoid arthritis, a 6-month, double-blind trial. Bull Rheum Dis 1962; 13: 287-90.

${ }^{141}$ Manny N, Roseman E, Benbassat J. Hazards of immunosuppressive therapy. Br Med J 1972; ii: 291.

${ }^{142}$ Martin PJ, Najfeld V, Hansen JA, Penfold GK, Jacobson RJ, Fialkow PJ. Involvement of the B-lymphoid system in chronic myelogenous leukaemia. Nature 1980; 287: 49-50.

${ }^{143}$ Mason RM, Currey HLF, Barnes CG, Dunne JF, Hazleman BL, Strickland ID. Azathioprine in rheumatoid arthritis. Br Med J 1969; i: 420-1.

${ }^{144}$ Melnyk J, Duffy DM, Sparkes RS. Human mitotic and 
meiotic chromosome damage following in vivo exposure to methotrexate. Clin Genet 1971; 2: 28.

${ }^{145}$ Menkes CJ, Levy JP, Weill B, Delrien F, Mathiot C, Delbarre $F$. Leucémie aigue à mégacaryoblasts survenue après traitement immunodepresseur d'une polyarthrite rhumatöide. Nouv Press Med 1975; 4: 2869.

${ }^{146}$ Michlmayr G, Gunther R, Lederer B, Huber H. Malignes lymphom nach zweijahriger immunosuppressiver therapie einer periarteritis nodosa. Med Klin 1973; 68: 180.

${ }^{147}$ Mielent H, Veys EM. A study of hemotologic side effects of levamisole in rheumatoid arthritis with recommendations. J Rheumatol 1978; 5: 4.

${ }^{148}$ Mikkelsen WM. The epidemiology of rheumatic diseases. In: Hollander JL, McCarty DJ Jr, eds. Arthritis and allied conditions. Philadelphia: Kimpton, 1972: 211.

${ }^{149}$ Miller DG. The association of immune disease and malignant lymphoma. Ann Intern Med 1967; 66: 507-21.

${ }^{150}$ Moens C, Brocteur J. Treatment of rheumatoid arthritis with immunosuppressive drugs. I. Clinical study. Acta Rheum Scand 1965; 11: 212-20.

${ }^{151}$ Moesman G. Malignancy and mortality in subacute rheumatoid arthritis in old age. Acta Rheum Scand 1969; 15: 193-9.

${ }^{152}$ Monson RR, Hall AP. Mortality among arthritics. $J$ Chron Dis 1976; 29: 459-67.

${ }^{153}$ Moorhead PS, Nowell PC, Mellman WJ. Chromosome preparation of leukocytes cultured from human peripheral blood. Exp Cell Res 1960; 20: 613.

${ }_{154}$ Mornington B. Nebenwirkungen von azathioprine beim Menschen. Vortrag des 10 e Europaischen Kongresses für Allergologie und Klinische Immunologie, September 1977, Prag.

${ }^{155}$ Multicentre Trial Group. Controlled trial of d-penicillamine in severe rheumatoid arthritis. Lancet 1973; i: $275-80$.

${ }^{156}$ Mutru O, Koota K, Isomaki H. Causes of death in autopsied RA patients. Scand J Rheumatol 1976; 5: 239-40.

${ }^{157}$ Neuberg R. Immunosuppression and plasmocytoma of the cervix. J Obstet Gynaecol British Commonwealth 1974; 81: 165-7.

${ }^{158}$ Neuhaus K, Thornhorst J, Bertel O, Speck S. Mehrfachtumoren unter Azathioprin Therapie bei progredient-chronischer Polyarthritis. Praxis 1976; 65: 213-21.

${ }^{159}$ Nies K, Boyer R, Stevens R, Louie J. Anti-tetanus toxoid antibody synthesis after booster immunization in systemic lupus erythematosus. Arthritis Rheum 1980; 23: 1343-50.

${ }^{160}$ Nossal GJV. Immunological tolerance: cellular facts and clinical speculations. In: Advanced medicine. Vol 16. Tunbridge Wells: Pitman Medical, 1980: 356-70.

${ }^{161}$ Oleinick A. Leukemia or lymphoma occurring subsequent to an autoimmune disease. Blood 1967; 29: 144-53.

${ }^{162}$ Ontario Cancer Treatment and Research Foundation, personal communication.
${ }^{163}$ Osborne WRA. Inherited absences of purine recycling enzymes associates with defects of immunity. Trends Biochem Sci 1981; 6: 80-3.

${ }^{164}$ Ouchterlony O. Antigen-antibody reactions in gels. Acta Pathol Microbiol Scand 1949; 26: 507-15.

${ }^{165}$ Overledenen naar doodsoorzaak, leeftijd en geslacht in het jaar 1973-1974-1975. CBS Hoofdafdeling Gezondheidsstatistieken.

${ }^{166}$ Owen DS, Waller M, Toone E. Rheumatoid disease and malignancy. Arthritis Rheum 1967; 10: 302.

${ }^{167}$ Owen DS Jr, Waller M, Toone EC Jr. Rheumatoid arthritis and malignancy. Med Coll Virginia $Q 1970$; 6: 8-10.

${ }^{168}$ Parker D, Dwyer JM, Turk JL. The effect of cyclophosphamide and the role of suppressor cells in the desensitization of delayed hypersensitivity. Immunology 1981; 43: 191-6.

${ }^{169}$ Parsons JL, Strong JS, Fosdick WM. The causes of death in patients with rheumatoid arthritis treated with cytotoxic agents. J Rheumatol 1974; 1, (suppl 1): 75.

170 Pätiälä H, Isomaki H, Laurinkari J. Rheumatoid arthritis and totally reimbursable medicines (in Finnish). Sosiaalilaaket Aikak 1975; 12: 584-7.

${ }^{171}$ Patterson JF, Norton RA, Schwartz RS. Azathioprine treatment of ulcerative colitis, granulomatous colitis and regional enteritis. Am J Dig Dis 1971; 16: 327.

${ }^{172}$ Pelton BK, Hylton W, Denman AM. A selective defect in B cell function in SLE. Clin Exp Immunol (in press).

${ }^{173}$ Pelton BK, Duncan IB, Denman AM. Herpes simplex virus depresses antibody production by affecting $\mathrm{T}$-cell function. Nature 1980; 284: 176-7.

174 Penn I. Malignancies associated with immunosuppressive or cytotoxic therapy. Clinical review. Surgery 1978; 83: 492-502.

175 Penn I, Starzl TE. A summary of the status of de novo cancer in transplant recipients. Transplant Proc 1972; 4: 719.

${ }^{176}$ Penn I, Starzl TE. Immunosuppression and cancer. Transplant Proc 1973; 5: 943.

${ }^{177}$ Pinals RS. Azathioprine in the treatment of chronic polyarthritis: long-term results and adverse effects in 25 patients. J Rheumatol 1976; 3: 140-4.

${ }^{178}$ Plotz PH, Klippel JH, Decker JL. Bladder complications in patients receiving cyclophosphamide for rheumatoid arthritis (RA) or systemic lupus erythematosus (SLE). Arthritis Rheum 1979; 22: 647 (abst).

${ }^{179}$ Pollock BH, Barr JH, Stolzer BL, Eisenbeis CH, Agarwal A, Margolis HM. Neoplasia and cyclophosphamide. Arthritis Rheum 1973; 16: 524.

${ }^{180}$ Popert AJ, Meijers KAE, Sharp J, Bier F. Chloroquine diphosphate in rheumatoid arthritis: a controlled trial. Ann Rheum Dis 1961; 20: 18-35.

${ }^{181}$ Rabkin R, Thatcher GN, Diamond LH, Eales L. The nephrotic syndrome, malignancy and immunosuppression. S Afr Med J 1973; 47: 605.

${ }^{182}$ Rasker JJ, Cosh JA. Cause and age of death in a prospective study of 100 patients with rheumatoid arthritis. Ann Rheum Dis 1981; 40: 115-20.

${ }^{183}$ Raven JL, Walker PL, Barkhan P. Comparison of the radioisotope dilution-coated charcoal method and a 
microbiological method (L. Leichmannii) for measuring vitamin B12 in serum. J Clin Pathol 1966; 19: 610.

${ }^{184}$ Renier J, Bregeon C, Barnette C, et al. Le devenier des sujets atteints de polyarthrite rhumatoide et traites parles immunodépresseurs entre 1965 et 1973 inclus. Rev Rhum 1978; 45: 453-61.

${ }^{185}$ Richter JA, Runge LA, Pinals RS. Analysis of treatment terminations with gold and antimalarial compounds in rheumatoid arthritis. J Rheumatol 1980; 7: 153-9.

${ }^{186}$ Ropes MW, Bennet GA, Cobb S, Jacox RF, Jessar RA. Revision of diagnostic criteria for rheumatoid arthritis. Bull Rheum Dis 1958; 9: 175-6.

${ }^{187}$ Rosner F, Grunwalk H. Multiple myeloma terminating in acute leukemia. Report of 12 cases and review of the literature. Am J Med 1974; 57: 927.

${ }^{188}$ Rothermich NO. Chrysotherapy in rheumatoid arthritis. Clin Rheum Dis 1979; 5: 631-40.

${ }^{189}$ Rothermich NO. Philips VK, Bergen W, Thomas MH. Followup study of chrysotherapy. Arthritis Rheum 1979; 22: 423.

190 Rynes RI, Krokel G, Falbo A, Reincke RD, Wolfe B, Bartholomew LE. Ophthalmologic safety of long-term hydroxychloroquine treatment. Arthritis Rheum 1979; 22: 832-6.

191 Savage O. Measurement in rheumatoid arthritis. Proc $R$ Soc Med 1966; 59, (suppl): 85.

${ }^{192}$ Scharf J, Nahir N, Eidelman S, Jacobs R, Levin D. Carcinoma of the bladder with azathioprine therapy. JAMA 1977; 237: 152.

${ }^{193}$ Schwartz RS, Cisner A, Dameshek W. The effect of 6-mercaptopurine on primary and secondary immune responses. J Clin Invest 1959; 38: 1394-403.

${ }^{194}$ Schwartz RS, Dameshek W. Drug-induced immunological tolerance. Nature 1959; 183: 1682-3.

195 Seabright M. A rapid banding technique for human chromosomes. Lancet 1971; ii: 971-2.

196 Seidenfeld AM, Smythe HA, Ogryzlo MA, Urowitz $\mathrm{MB}$, Dotton DA. Acute leukaemia in rheumatoid arthritis treated with cytotoxic agents.J Rheumatol 1976; 3: 295-304.

${ }^{197}$ Shand FL. The immunopharmacology of cyclophosphamide. Int J Immunopharmacol 1979; 1: 165-71.

${ }^{198}$ Sharon E, Greenwalk R, Solish G, Diamond H, Kaplan $D$. Adverse effects of azathioprine (Az) in patients with systemic lupus erythematosus (SLE). Clin Pharmacol Ther 1972; 13: 152.

199 Sharpstone P, Cameron JS. Nephrotic syndrome due to primary renal disease in adults. II. A controlled trial of prednisone and azathioprine. Br Med J 1969; ii: 535.

${ }^{200}$ Silvergleid AJ, Schrier SL. Acute myelogenous leukemia in two patients treated with azathioprine for nonmalignant diseases. Am J Med 1974; 57: 885.

${ }^{201}$ Singer JM, Plotz CM. The latex fixation test. Application to the serologic diagnosis of rheumatoid arthritis. Am J Med 1956; 21: 888.

${ }^{202}$ Skinner MD, Schwartz RS. Immunosuppressive therapy. N Engl J Med 1972; 287: 221.

${ }^{203}$ Slater A, Whittaker JA, Fisher OJH. Reticulum cell sarcoma and Sjögren's syndrome in a patient treated with azathioprine. New Engl J Med 1976; 294: 51.
${ }^{204}$ Spray GH. Estimation of red cell folate activity. J Clin Pathol 1969; 22: 212.

${ }^{205}$ Srinivasan R, Miller BL, Paulus HE. Longterm chrysotherapy in RA. Arthritis Rheum 1979; 22: 105-10.

${ }^{206}$ Swannell ȦJ, Coomes EN. Preliminary results of azathioprine treatment in severe rheumatic disease. Ann Phys Med 1969; 10: 112-20.

${ }^{207}$ Swannell AJ, Kersley GD. Preliminary results with azathioprine treatment in rheumatoid arthritis. Ann Phys Med 1969; 10: 163-6.

${ }^{208}$ Symington GR, Mackay IR, Lambert RP. Cancer and teratogenesis: infrequent occurrence after medical use of immunosuppressive drugs. Aust NZ J Med 1977; 7: 368-72.

${ }^{209}$ Symoens J, Schuermans Y. Levamisole. Clin Rheum Dis 1979; 5: 603-29.

${ }^{210}$ Talal N, ed. Autoimmunity. London: Academic Press, 1980.

${ }^{211}$ Talal N, Bunim JJ. The development of malignant lymphomata in the course of Sjögren's syndrome. Am J Med 1964; 36: 529-40.

${ }^{212}$ Tausch G, Eberl R, Siegmeth W, Sochor H. Long-term therapy of chronic progressive polyarthritis with azathioprine. $Z$ Rheumaforsch 1970; 29: 162-7 (translation).

${ }^{213}$ Teppo L, Hakama M, Hakulinen T, et al. Cancer in Finland 1953-1970: incidence, mortality, prevalence. Acta Path Microbiol Scand 1975; suppl 252.

${ }^{214}$ Thorpe P, Hasall JE, York JR. Rheumatoid arthritis treated with chlorambucil. Med J Aust 1976; 2: 197-9.

${ }^{215}$ Tjio JH, Whang J. Chromosome preparations of bone marrow cells without prior in vitro culture or in vivo colchicine administration. Stain Techniques 1962; 37: 17.

216 Tolchin SF, Winkelstein A, Rodnan GP, Pan SF, Nankin HR. Chromosome abnormalities from cyclophosphamide therapy in rheumatoid arthritis and progressive systemic sclerosis (scleroderma). Arthritis Rheum 1974; 17: 375-82.

${ }^{217}$ Turk JL. Immunology of infections. In: Dick G, ed. Immunological aspects of infectious diseases. Lancaster: MTP Press, 1979: 421-52.

218 Turnbull A, Almeyda J. Idiopathic thrombocytopenic purpura and Kaposi's sarcoma. Proc R Soc Med 1970; 63: 603 .

${ }^{219}$ Uddin J, Kraus AS, Kelly HG. Survivorship and death in rheumatoid arthritis. Arthritis Rheum 1970; 13: 125-31.

${ }^{220}$ Uhl GS, Williams JE, Arnett FC. Intracerebral lymphomas in a patient with central nervous system lupus on cyclophosphamide. J Rheumatol 1974; 1: 282.

${ }^{221}$ Ulrich J, Wüthrich R. Multiple sclerosis: reticulum cell sarcoma of the nervous system in patient treated with immunosuppressive drugs. Eur Neurol 1974; 12: 65-8.

${ }^{222}$ Unger A, Panayi GS, Lessof MH. Carcinoembryonic antigen in rheumatoid arthritis. Lancet 1974; 1: 781.

${ }^{223}$ Unger A, Panayi GS, Lessof MH. Carcinoembryonic antigen in rheumatic diseases. Rheumatol Rehabil 1975; $14: 19$.

${ }^{224}$ Urowitz MB, Gordon DA, Smythe HA, Pruzanski W, 
Ogryzlo MA. Azathioprine in rheumatoid arthritis. A double-blind crossover study. Arthritis Rheum 1973; 16: 411-8.

225 Urowitz MB, Hunter T, Bookman AAM, Gordon DA, Smythe HA, Ogryzlo MA. Azathioprine in rheumatoid arthritis: a double-blind study comparing full dose to half dose. J Rheumatol 1974; 1: 274-81.

${ }^{226}$ Van Dam G, Lezwijn A, Bos JG. Death rate of patients with rheumatoid arthritis. In: Atti del Congresso del la Lega Internationale Contro il Rheumatismo Roma, Melazioni, Torino. Minerva Medica 1961: 2.

${ }^{227}$ Vandenbroucke JP, Kok FJ, Matroos A, Dekker E. Rookgewoonten van Nederlandse huisartsen vergeleken met die van de bevolking. Ned Tijdschr Geneeskd 1981; 1: 4-7.

${ }^{228}$ Verhaegen H, DeCock W, DeCree J. The effects of azathioprine and levamisole on rosette-forming cells of healthy subjects and cancer patients. Clin Exp Immunol 1977; 29: 311-5.

${ }^{229}$ Veys EM, Mielants H, Verbruggen G, Dhondt E, Goethals L, Cheroutre L, Buelens H. Levamisole as basic treatment of rheumatoid arthritis: long-term evaluation. J Rheumatol 1981; 8: 45-56.

${ }^{230}$ von Vormittag W. Zytostatische immunodepressive Therapie, chromosomale Aberrationen und karzinogene Wirkung. Wien Klin Wochenschr 1974; 3: 69-75.

${ }^{231}$ Walder BK, Robertson MR, Jeremy D. Skin cancer and immunosuppression. Lancet 1971; ii: 1282-3.

${ }^{232}$ Wall RL, Clausen KP. Carcinoma of the urinary blad- der in patients receiving cyclophosphamide. $N$ Engl $J$ Med 1975; 293: 271-3.

${ }^{233}$ Webley M, Coomes EN. An assessment of penicillamine therapy in rheumatoid arthritis and the influence of previous gold therapy. J Rheumatol 1979; 6: $20-4$.

${ }^{234}$ Wegelius O, Skrifvars B, Andersson L. Rheumatoid arthritis terminating in plasmocytoma. Acta Med Scand 1970; 187: 133-8.

${ }^{235}$ Weinstein A. Drug-induced systemic lupus erythematosus. In: Schwartz RS, ed. Progress in clinical immunology. Vol 4. New York: Grune and Stratton, 1980: 1-22.

${ }^{236}$ Wheeler GE. Cyclophosphamide-associated leukemia in Wegener's granulomatosis. Ann Intern Med 1981; 94: 361 .

${ }^{237}$ Williams HJ, Reading JC, Ward JR, O'Brien WM. Comparison of high and low dose cyclophosphamide therapy in rheumatoid arthritis. Arthritis Rheum 1980; 23: 521-7.

${ }^{238}$ Wishart J. Reticulosarcoma of vulva complicating azathioprine-treated dermatomyositis. Arch Dermatol 1973; $108: 563$.

${ }^{239}$ Woodland J, Chaput de Saintonge DM, Evans SJW, Currey HLF. Azathioprine in rheumatoid arthritis: double-blind study of full vs half dosage vs placebo. Ann Rheum Dis 1980; 39: 605-6.

${ }^{240}$ Worlledge SM, Brain MC, Cooper AC, Hobbs JR Dacie JV. Immunosuppressive drugs in the treatment of autoimmune haemolytic anaemia. Proc $R$ Soc Me 1968; 61: 1312 . 